\title{
UNIVERSALITY IN MEAN CURVATURE FLOW NECKPINCHES
}

\author{
ZHOU GANG AND DAN KNOPF
}

\begin{abstract}
We study noncompact surfaces evolving by mean curvature flow. Without any symmetry assumptions, we prove that any solution that is $C^{3}$ close at some time to a standard neck will develop a neckpinch singularity in finite time, will become asymptotically rotationally symmetric in a space-time neighborhood of its singular set, and will have a unique tangent flow.
\end{abstract}

\section{Contents}

1. Introduction

2. Preliminaries

3. How the solution evolves

4. The first bootstrap machine

5. Improved estimates for the inner region 14

6. The second bootstrap machine 23

7. Improved estimates for the decomposition 26

8. Proof of the Main Theorem 30

Appendix A. Proof of Lemma 9

Appendix B. Proof of Lemma $11 \quad 38$

Appendix C. Proof of Lemma $12 \quad 44$

$\begin{array}{ll}\text { References } & 50\end{array}$

\section{INTRODUCTION}

In this paper, we prove, without imposing any symmetry assumptions, that any complete noncompact two-dimensional solution of mean curvature flow (MCF) that is close to a standard round neck at some time will (I) encounter a finitetime singularity, (II) become asymptotically rotationally symmetric in a space-time neighborhood of the developing singularity, (III) satisfy an exact asymptotic profile in that neighborhood, and (IV) will as a consequence have a unique tangent flow. All of these statements are made precise below. This result extends our previous work on MCF singularities: in that work [13], we removed the hypothesis of rotational symmetry but retained certain discrete symmetry hypotheses that served to fix the limiting cylinder. The results in this paper, combined with significant work of others, makes it reasonable to expect that singularities of generic MCF solutions may be constrained to a small selection of "universal" asymptotic profiles. Before giving the details, of our results, we sketch the broad outlines of the emerging picture motivating this expectation.

ZG thanks NSF for support in DMS-1308985. DK thanks NSF for support in DMS-1205270. 
One says a smooth one-parameter family $\mathcal{M}_{t}^{m} \subset \mathbb{R}^{m+1}$ of hypersurfaces moves by MCF if at every point $x \in \mathcal{M}_{t}^{m}$, one has $\partial_{t} x=-H \nu$, where $H$ is the mean curvature scalar, and $\nu$ is the unit normal at $x$. Denote the backward heat kernel at $\left(x_{0}, t_{0}\right)$ by $\rho_{\left(x_{0}, t_{0}\right)}(x, t)=\left\{4 \pi\left(t_{0}-t\right)\right\}^{-m / 2} \exp \left\{-\left|x-x_{0}\right|^{2} / 4\left(t_{0}-t\right)\right\}$. For $t<t_{0}$, Huisken's MCF monotonicity formula [17] states that

$$
\frac{d}{d t} \int_{\mathcal{M}_{t}^{m}} \rho_{\left(x_{0}, t_{0}\right)}(x, t) \mathrm{d} \mu=-\int_{\mathcal{M}_{t}^{m}}\left|\frac{\left(x-x_{0}\right)^{\perp}}{2\left(t_{0}-t\right)}-H \nu\right|^{2} \rho_{\left(x_{0}, t_{0}\right)}(x, t) \mathrm{d} \mu .
$$

A consequence of this is the following characterization of tangent flows (singularity models) for Type-I MCF singularities:

Theorem A (Huisken). Given any sequence of parabolic dilations at a Type-I $\mathrm{MCF}$ singularity, there exists a subsequence that converges smoothly to a nonempty immersed self-similarly shrinking solution.

In important subsequent work, Huisken and Sinestrari prove that any mean convex solution becomes asymptotically convex at its first singular time [18], and they develop a complete surgery program for solutions originating from two-convex initial data $\mathcal{M}_{0}^{m} \subset \mathbb{R}^{m+1}$ that are immersed images of compact manifolds of dimensions $m \geq 3$ [19]. In the latter work, they show in particular that all singularities of such solutions are either spherical $\left(\mathbb{S}^{m}\right)$ or neckpinch $\left(\mathbb{R} \times \mathbb{S}^{m-1}\right)$ singularities. Subsequently, Brendle and Huisken [5] extend this result to MCF with surgery of mean-convex initial surfaces $\mathcal{M}_{0}^{2} \subset \mathbb{R}^{3}$. Very recently, Haslhofer and Kleiner [16] obtain similar results for MCF with surgery, without dimension restrictions, using shorter proofs that rely on blow-up arguments.

Also recently, given $t_{0}>0$ and a hypersurface $\mathcal{M}^{m} \subset \mathbb{R}^{m+1}$, Colding and Minicozzi consider a functional $F_{\left(x_{0}, t_{0}\right)}\left(\mathcal{M}^{m}\right)=\int_{\mathcal{M}^{m}} \rho_{\left(x_{0}, t_{0}\right)}(\cdot, 0) \mathrm{d} \mu$, and an entropy $\lambda\left(\mathcal{M}^{m}\right)=\sup _{\left(x_{0}, t_{0}\right)} F_{\left(x_{0}, t_{0}\right)}\left(\mathcal{M}^{m}\right)$. The entropy $\lambda$ is invariant under dilations and Euclidean motions, and $\lambda\left(\mathcal{M}_{t}^{m}\right)$ is nonincreasing under MCF. Among other results, they prove a stability property for tangent flows [8].

Theorem B (Colding-Minicozzi). Let $\mathcal{M}^{m} \subset \mathbb{R}^{m+1}$ be a smooth ${ }^{1}$ complete embedded self-shrinker with polynomial volume growth and without boundary.

If $\mathcal{M}^{m}$ is not equal to any $\mathbb{S}^{k} \times \mathbb{R}^{m-k}, 0 \leq k \leq m$, then for any fixed $r$, there is a graph $\mathcal{N}^{m}$ over $\mathcal{M}^{m}$ of a function with arbitrarily small $C^{r}$ norm such that $\lambda\left(\mathcal{N}^{m}\right)<\lambda\left(\mathcal{M}^{m}\right)$.

A consequence of Theorem $\mathrm{B}$ is that $\mathcal{M}^{m}$ cannot occur as a tangent flow of a MCF solution whose initial data are any perturbations $\mathcal{N}^{m}$ of $\mathcal{M}^{m}$. It follows that spheres and cylinders are the only generic self-similarly shrinking tangent flows.

Left open by these important results is the question of whether singularity models are independent of subsequence. This has been answered affirmatively by Schulze [23] (using the Simon-Łojasiewicz inequality) if one tangent flow is a closed, multiplicity-one, smoothly embedded self-similar shrinker, but the general case remains open. Progress toward resolving this question was recently made by Colding, Ilmanen, and Minicozzi [7], who prove that if one tangent flow at a singularity is a

\footnotetext{
${ }^{1}$ Recall that by Ilmanen's $\mathbb{R}^{3}$ blowup theorem [21], a compact surface evolving by MCF has a smooth singularity model at its first singular time. In dimensions $3 \leq m \leq 6$, Theorem $\mathrm{B}$ also holds if $\mathcal{M}^{m}$ is merely smooth away from a singular set of locally finite $(m-2)$-dimensional Hausdorff measure.
} 
multiplicity-one generalized cylinder, then every subsequential limit is some generalized cylinder. Since this paper was written, its authors learned of important work of Colding and Minicozzi [9], who prove that tangent cones are unique for generic singularities of MCF, and for all singularities of mean-convex MCF. Other recent progress towards classifying singularity models comes from work of Wang [24], who proves that there is at most one smooth complete properly embedded self-shrinker asymptotic at spatial infinity to any regular cone.

In this paper, we show that the tangent cylinders of certain MCF neckpinches are independent of subsequence by proving that these singularities have unique asymptotic behavior. With few exceptions (i.e. [11] for logarithmic fast diffusion) asymptotic analysis results of this nature (e.g. [22] and [10] for logarithmic fast diffusion; [3], [1], and [2] for Ricci flow; and [4] and [14] for MCF) require a restrictive hypothesis of rotational symmetry. In [13], with I.M. Sigal, we made partial progress towards showing uniqueness by removing the hypothesis of rotational symmetry for MCF neckpinches, retaining only weaker discrete symmetries, and then proving that neckpinches with these discrete symmetries asymptotically become rotationally symmetric. (See [13] for precise statements of these assumptions and results.) Here, we extend that work by removing symmetry assumptions altogether. We show that neckpinches originating from initial data sufficiently close to a formal solution develop unique asymptotic profiles, modulo dilations and Euclidean motions.

At least if Ilmanen's multiplicity conjecture [20] is true, then combining our work here with the results cited above makes it reasonable to conjecture that MCF solutions originating from generic initial data $\mathcal{M}_{0}^{2} \subset \mathbb{R}^{3}$ are constrained to only two universal asymptotic profiles if they become singular.

Passing to higher dimensions, we strongly conjecture that our main results in this paper generalize to hypersurface neckpinches $\mathcal{N}_{t}^{m} \subset \mathbb{R}^{m+1}$ in all dimensions. Indeed, the strategy of the proof, as outlined in Section 2.3, rests on the fact that although the linearization of MCF at a cylinder $\mathbb{R} \times \mathbb{S}^{1}$ is formally unstable, the unstable eigenmodes are simply "coordinate instabilities." By properly choosing seven coordinate parameters, a solution is decomposed into a dominant component, and a rapidly-decaying component on which the linearization is strictly stable. The only complications in extending these ideas to dimensions $m>2$ are that one must deal with $m+5$ coordinate parameters, and with curvature terms that arise from commuting covariant derivatives on $\mathbb{S}^{m-1}$. The latter, however, are harmless, because the coordinate parametrization controls the size of the cylinder.

This paper is structured as follows. As in [13], we prove our results by means of two bootstrap machines. In Section 2, we establish notation, state our main assumptions, and outline the main ideas introduced here to generalize our earlier work. In Section 3, we derive the evolution equations for the quantities under analysis. We construct the first bootstrap machine in Sections $4-5$ and the second in Sections 6-7. We complete the proof of the Main Theorem in Section 8. The proofs of several supporting technical results appear in the appendices.

We now summarize the main results of this paper, using the following terminology. We say that $(x, r, \theta)$ is a cylindrical coordinate system if there exist orthonormal coordinates $\left(x_{0}, x_{1}, x_{2}\right)$ for $\mathbb{R}^{3}$ such that $x=x_{0}$ is the cylindrical axis and $(r, \theta)$ are polar coordinates for the $\left(x_{1}, x_{2}\right)$-plane. In this notation, the standard cylinder with axis $x$ is the set $\{r=1\}$, and a normal graph is determined by $r=u(x, \theta)$. 
Main Theorem. Suppose that a solution of MCF satisfies Assumptions [A1]-[A7] from Section 2.2 for sufficiently small $b_{0}, c_{0}$.

(I) There exists $T<\infty$ such that the solution becomes singular at time $T$.

(II) There exists a sequence $(x, r, \theta)_{n}$ of cylindrical coordinate systems such that for all times $t_{n-1} \leq t \leq t_{n}$, the surface can be written as the normal graph of a positive function $u(x, \theta, t)$. Moreover, $t_{n} \nearrow T$ as $n \rightarrow \infty$, and there exists a limiting $(x, r, \theta)_{n} \rightarrow(x, r, \theta)_{\infty}$ cylindrical coordinate system. In these coordinates, the solution develops a neckpinch, with $u(x, \cdot, t)=0$ if and only if $x=0$ and $t=T$.

(III) In the cylindrical coordinate system $(x, r, \theta)_{n}$ constructed at time $t_{n}$, the solution admits an "optimal coordinate" decomposition

$$
\frac{u\left(x, \theta, t_{n}\right)}{\lambda_{\mathrm{opt}}\left(t_{n}\right)}=\sqrt{\frac{2+b_{\mathrm{opt}}\left(t_{n}\right) y^{2}}{1+\frac{1}{2} b_{\mathrm{opt}}\left(t_{n}\right)}}+\phi_{\mathrm{opt}}\left(y, \theta, t_{n}\right),
$$

where $y=\lambda_{\mathrm{opt}}\left(t_{n}\right)^{-1} x$. The parameters $\lambda_{\mathrm{opt}}\left(t_{n}\right)$ and $b_{\mathrm{opt}}\left(t_{n}\right)$ have the asymptotic behavior that as $t_{n} \nearrow T$,

$$
\begin{aligned}
\lambda_{\text {opt }}\left(t_{n}\right) & =\{1+o(1)\} \sqrt{T-t_{n}}, \\
b_{\text {opt }}\left(t_{n}\right) & =\{1+o(1)\}\left(-\log \left(T-t_{n}\right)\right)^{-1} .
\end{aligned}
$$

(IV) The solution is asymptotically rotationally symmetric near the singularity in the precise technical senses that there exist $\varepsilon_{1}, \varepsilon_{2}>0$ such that in each cylindrical coordinate system $(x, r, \theta)_{n}$ at all times $t \in\left[t_{n-1}, t_{n}\right]$, there exist $C^{1}$ functions $\lambda$, $a, b$, and $\beta_{0}, \ldots, \beta_{4}$ of time, with $\lambda\left(t_{n}\right)=\{1+o(1)\} \lambda_{\text {opt }}\left(t_{n}\right), a\left(t_{n}\right)=\{1+o(1)\} \frac{1}{2}$, $b\left(t_{n}\right)=\{1+o(1)\} b_{\text {opt }}\left(t_{n}\right)$, and $\left|\beta_{k}(t)\right| \lesssim(-\log (T-t))^{-2}$ for $k=0, \ldots, 4$, such that the $\theta$-dependent component $\phi$ of the solution defined by

$$
\phi:=\frac{u}{\lambda}-\sqrt{\frac{2+b y^{2}}{2-2 a}}-\beta_{0} y-\beta_{1} \cos \theta-\beta_{2} \sin \theta-\beta_{3} y \cos \theta-\beta_{4} y \sin \theta
$$

satisfies the derivative decay estimates

$$
v^{-2}\left|\partial_{\theta} \phi\right|+v^{-1}\left|\partial_{y} \partial_{\theta} \phi\right|+v^{-2}\left|\partial_{\theta}^{2} \phi\right|+v^{-1}\left|\partial_{y}^{2} \partial_{\theta} \phi\right|+v^{-2}\left|\partial_{y} \partial_{\theta}^{2} \phi\right|+v^{-3}\left|\partial_{\theta}^{3} \phi\right| \lesssim b(t)^{2-\varepsilon_{1}},
$$

and the $C^{0}$ decay estimates

$$
\frac{|\phi(y, \theta, t)|}{\left(1+y^{2}\right)^{\frac{3}{2}}} \lesssim b(t)^{2-\varepsilon_{2}} \quad \text { and } \quad \frac{|\phi(y, \theta, t)|}{\left(1+y^{2}\right)^{\frac{11}{20}}} \lesssim b(t)^{1-\varepsilon_{1}}
$$

all holding uniformly as $t_{n} \nearrow T$.

Acknowledgment. The authors are deeply grateful to I.M. Sigal for many helpful discussions related to extensions of their work in [13].

\section{Preliminaries}

2.1. Notation. We study the evolution of embedded graphs over a cylinder $\mathbb{S}^{1} \times \mathbb{R}$ in $\mathbb{R}^{3}$. We consider initial data $\mathcal{M}_{0}$ expressed in a cylindrical coordinate system by a positive function $u_{0}(x, \theta)$. Then for as long as the flow remains a graph, all $\mathcal{M}_{t}$ are determined by $r=u(x, \theta, t)$, where, as we showed in [13], $u$ satisfies the initial condition $u(x, \theta, 0)=u_{0}(x, \theta)$ and evolves by

$$
\partial_{t} u=\frac{\left\{1+\left(\frac{\partial_{\theta} u}{u}\right)^{2}\right\} \partial_{x}^{2} u+\frac{1+\left(\partial_{x} u\right)^{2}}{u^{2}} \partial_{\theta}^{2} u-2 \frac{\left(\partial_{x} u\right)\left(\partial_{\theta} u\right)^{2}}{u^{3}} \partial_{x} \partial_{\theta} u-\frac{\left(\partial_{\theta} u\right)^{2}}{u^{3}}}{1+\left(\partial_{x} u\right)^{2}+\left(\frac{\partial_{\theta} u}{u}\right)^{2}}-\frac{1}{u} .
$$


As in [13], we apply adaptive rescaling, transforming the original space-time variables $x$ and $t$ into rescaled blowup variables ${ }^{2}$

$$
y(x, t):=\lambda^{-1}(t) x \quad \text { and } \quad \tau(t):=\int_{0}^{t} \lambda^{-2}(s) \mathrm{d} s,
$$

where $\lambda>0$ is a scaling parameter to be chosen, with $\lambda(0)=\lambda_{0}$. The equation's scaling symmetry allows us, without loss of generality, to fix $\lambda_{0}=1$. Note that $y=0$ is only the approximate center of the developing neckpinch; we address this issue below. We define a rescaled radius $v(y, \theta, \tau)$ by

$$
v(y(x, t), \theta, \tau(t)):=\lambda^{-1}(t) u(x, \theta, t) .
$$

Then $v$ initially satisfies $v(y, \theta, 0)=v_{0}(y, \theta)$, where $v_{0}(y, \theta):=\lambda_{0}^{-1} u_{0}\left(\lambda_{0} y, \theta\right)$.

With respect to commuting $(y, \theta, \tau)$ derivatives, the quantity $v$ evolves by

$$
\partial_{\tau} v=A_{v} v+a v-v^{-1},
$$

where $A_{v}$ is the quasilinear elliptic operator

$$
A_{v}:=F_{1}(p, q) \partial_{y}^{2}+v^{-2} F_{2}(p, q) \partial_{\theta}^{2}+v^{-1} F_{3}(p, q) \partial_{y} \partial_{\theta}+v^{-2} F_{4}(p, q) \partial_{\theta}-a y \partial_{y} .
$$

The coefficients of $A_{v}$ are defined by

$$
\begin{array}{lll}
F_{1}(p, q):=\frac{1+q^{2}}{1+p^{2}+q^{2}}, & F_{2}(p, q):=\frac{1+p^{2}}{1+p^{2}+q^{2}}, \\
F_{3}(p, q):=-\frac{2 p q}{1+p^{2}+q^{2}}, & F_{4}(p, q):=\frac{q}{1+p^{2}+q^{2}},
\end{array}
$$

where

$$
a:=-\lambda \partial_{t} \lambda, \quad p:=\partial_{y} v, \quad \text { and } \quad q:=v^{-1} \partial_{\theta} v .
$$

Before stating our assumptions, we require some further notation. We denote the formal solution of the adiabatic approximation to equation (2.3) by

$$
V_{r, s}(y):=\sqrt{\frac{2+s y^{2}}{2-2 r}},
$$

where $r$ and $s$ are positive parameters. We introduce a step function

$$
g(y, s):=\left\{\begin{array}{ccc}
\frac{19}{20} \sqrt{2} & \text { if } & s y^{2}<20 \\
4 & \text { if } & s y^{2} \geq 20 .
\end{array}\right.
$$

This function differs from that used in [13] in that we here prescribe a slightly sharper constant in the inner region $\left\{\beta y^{2} \leq 20\right\}$, where

$$
\beta(\tau):=\left(\kappa_{0}+\tau\right)^{-1} \text {. }
$$

This modification is not essential but simplifies some of the extra work required to compensate for the lack of discrete symmetry assumptions in this paper. ${ }^{3}$

\footnotetext{
${ }^{2}$ In the sequel, we abuse notation by using whichever time scale $(t$ or $\tau$ ) is most convenient.

${ }^{3}$ Here, $\kappa_{0} \gg 1$ is a large constant to be fixed below. For simplicity, we may without loss of generality set $\kappa_{0} \equiv b_{0}^{-1}$, where $b_{0} \ll 1$ is the constant introduced in Section 2.2.
} 
We introduce a Hilbert space $L_{\mu}^{2} \equiv L^{2}\left(\mathbb{S}^{1} \times \mathbb{R} ; \mathrm{d} \theta \mu \mathrm{d} y\right)$, with weighted measure $\mu(y):=\left(M+y^{2}\right)^{-\frac{3}{5}}$ defined with respect to a constant $M \gg 1$ to be fixed below. ${ }^{4}$ We denote the $L_{\mu}^{2}$ inner product by

$$
\langle\varphi, \psi\rangle_{\mu}:=\int_{\mathbb{R}} \int_{\mathbb{S}^{1}} \varphi \psi \mathrm{d} \theta \mu \mathrm{d} y,
$$

and its norm by $\|\cdot\|_{\mu}$. The inner product in the complex Hilbert space $L^{2}\left(\mathbb{S}^{1}\right)$ is denoted by

$$
\langle\varphi, \psi\rangle_{\mathbb{S}^{1}}:=\int_{\mathbb{S}^{1}} \varphi \psi \mathrm{d} \theta
$$

and its norm is $\|\cdot\|_{\mathbb{S}^{1}}$. The undecorated inner product $\langle\cdot, \cdot\rangle$ denotes the standard (unweighted) inner product in $L^{2}\left(\mathbb{S}^{1} \times \mathbb{R}\right)$.

We define a norm $\|\cdot\|_{m, n}$ by

$$
\|\phi\|_{m, n}:=\left\|\left(1+y^{2}\right)^{-\frac{m}{2}} \partial_{y}^{n} \phi\right\|_{L^{\infty}} .
$$

We write $\varphi \lesssim \psi$ if there exists a uniform constant $C>0$ such that $\varphi \leq C \psi$, and we set $\langle x\rangle:=\sqrt{1+|x|^{2}}$. Finally, for any $f(y, \theta, \tau)$, we define

$$
f_{ \pm}(y, \tau):=\frac{1}{2 \pi}\left\langle f(y, \theta, \tau), e^{ \pm i \theta}\right\rangle_{\mathbb{S}^{1}} .
$$

2.2. Main Assumptions. There are small positive constants $b_{0}, c_{0}$ such that:

[A1] The initial surface is a graph over $\mathbb{S}^{1} \times \mathbb{R}$ determined by a smooth function $u_{0}(x, \theta)>0$. The function $u_{0}$ is uniformly $\left(2 b_{0}\right)$-Lipschitz and satisfies $u_{0}(x, \cdot) \geq c_{*} V_{a_{0}, b_{0}}(x)$ for some $c_{*}>0$, along with the estimates ${ }^{5}$

$$
\begin{aligned}
\left|\left(u_{0}\right)_{ \pm}\right|+\left|\left(\partial_{x} u_{0}\right)_{ \pm}\right| & <b_{0}^{2}, \\
\left\|\langle x\rangle^{-5} \partial_{\theta} u_{0}\right\|_{L^{\infty}} & <b_{0}^{21 / 10}, \\
\left\|\langle x\rangle^{-11 / 10}\left(u_{0}\right)_{ \pm}\right\|_{L^{\infty}}+\left\|\langle x\rangle^{-11 / 10}\left(\partial_{x} u_{0}\right)_{ \pm}\right\|_{L^{\infty}} & <b_{0}^{53 / 20} .
\end{aligned}
$$

[A2] The initial function satisfies $u_{0}(x, \cdot)>g\left(x, b_{0}\right)$.

[A3] The initial surface is a small deformation of a formal solution $V_{a_{0}, b_{0}}(x)$ in the sense that for $(m, n) \in\{(3,0),(11 / 10,0),(2,1),(1,1)\}$, one has

$$
\left\|u_{0}(\cdot)-V_{a_{0}, b_{0}}(\cdot)\right\|_{m, n}<b_{0}^{\frac{m+n}{2}+\frac{1}{10}} .
$$

[A4] The parameter $a_{0}=a(0)$ obeys the bound $\left|a_{0}-1 / 2\right|<c_{0}$.

[A5] The initial surface obeys the further pointwise derivative bounds

$$
\begin{aligned}
& \sum_{n \neq 0,2 \leq m+n \leq 3} u_{0}^{-n}\left|\partial_{x}^{m} \partial_{\theta}^{n} u_{0}\right|<b_{0}^{2}, \\
& b_{0} u_{0}^{-1 / 2}\left|\partial_{x} u_{0}\right|+b_{0}^{1 / 2}\left|\partial_{x}^{2} u_{0}\right|+\left|\partial_{x}^{3} u_{0}\right|<b_{0}^{\frac{3}{2}}, \\
&\left|\partial_{x} \partial_{\theta}^{2} u_{0}\right|+u_{0}^{-1}\left|\partial_{\theta}^{3} u_{0}\right|<c_{0} .
\end{aligned}
$$

[A6] The initial surface obeys the Sobolev bounds

$$
b_{0}^{4 / 5}\left\|\partial_{x}^{4} u_{0}\right\|_{\mu}+\left\|\partial_{x}^{5} u_{0}\right\|_{\mu}+\sum_{n \neq 0,4 \leq m+n \leq 5}\left\|u_{0}^{-n} \partial_{x}^{m} \partial_{\theta}^{n} u_{0}\right\|_{\mu}<b_{0}^{4} .
$$

[A7] The initial surface satisfies $\left\|u_{0}^{-n} \partial_{x}^{m} \partial_{\theta}^{n} u_{0}\right\|_{L^{\infty}}<\infty$ for $4 \leq m+n \leq 6$.

\footnotetext{
${ }^{4}$ In [13], $\mu$ and $M$ were denoted by $\sigma$ and $\Sigma$, respectively.

${ }^{5}$ Smallness of $\left(u_{0}\right)_{ \pm}$and $\left(\partial_{x} u_{0}\right)_{ \pm}$in the inner region suffice for the first bootstrap machine, but global smallness is needed for certain propagator estimates in the second bootstrap machine.
} 
Three remarks are in order here: (I) Our choice $\lambda_{0}=1$ guarantees that $x=y$ and $\tau=0$ both hold at $t=0$, hence that the assumptions above apply identically to $u(x, \theta, 0)=u_{0}(x, \theta)$ and $v(y, \theta, 0)=v_{0}(y, \theta)$. (II) The lower bounds for $u_{0}$ in Assumptions [A1] and [A2] are clearly related, with [A1] being stronger for large $|x|$, and [A2] being stronger for small $|x|$. The bounds are presented in this way so that $[\mathrm{A} 1]$ is the only member of $[\mathrm{A} 1]-[\mathrm{A} 6]$ that is significantly changed from our earlier work [13], other than the slight modification of the function $g$ used in [A2]. The new inequalities in [A1] compensate for the removal of the discrete symmetries that were used in [13]; we explain this further below. (III) Assumption [A7], which is also new, imposes slightly more control at spatial infinity than we needed in [13]. This allows us to integrate by parts in $y$ without needing the hypothesis of reflection symmetry that we used in that earlier paper. (See Remark 2 below.)

2.3. Strategy of the proof. In earlier work [13] (also see [14]) we decomposed a solution $v(y, \theta, \tau)$ of equation (2.3) into two terms: a point $V_{a(\tau), b(\tau)}(y)$ on a manifold $\mathfrak{M}$ of approximate solutions, and a remainder term $\phi(y, \theta, \tau):=v(y, \theta, \tau)-$ $V_{a(\tau), b(\tau)}(y)$ approximately orthogonal to that manifold. These terms represent the large, slowly-changing part of the solution, and the small, rapidly-decaying part, respectively. The utility of this decomposition is that permutations of $v$ tangent to $\mathfrak{M}$ are linearly unstable, whereas those orthogonal to $\mathfrak{M}$ are linearly stable. We follow the same strategy here, except that our lack of symmetry assumptions requires us to construct a more refined decomposition in order to compensate for coordinate instabilities of (2.3). The details of this refinement are as follows.

In [13], we imposed discrete symmetries on the initial surface: $u_{0}(-x, \cdot)=u_{0}(x, \cdot)$ and $u_{0}(\cdot, \theta+\pi)=u_{0}(\cdot, \theta)$. These assumptions fixed the cylindrical axis and center $y=0$ of the developing neckpinch singularity. In the present paper, we remove these assumptions, which means that we must determine the unique cylinder and center at which the neckpinch forms. To compensate, Assumption [A1] is strengthened from [13]. Its revised form ensures that a neck aligned to a slightly perturbed cylinder can still be written as a normal graph, and provides extra estimates (to be improved in the second bootstrap machine) for the $\theta$-dependence of the solution. These estimates effectively replace the lost symmetries, which implied inequalities (like $\left\|\partial_{\theta}^{2} v\right\|_{\mathbb{S}^{1}} \geq 2\left\|\partial_{\theta} v\right\|_{\mathbb{S}^{1}}$ ) that we cannot use here.

We determine the unique cylinder and center of the neckpinch as follows. First, at some fixed time $\tau_{1}$, we construct "optimal coordinates" for the developing solution. As motivation, we observe that linearizing equation (2.3) at the cylinder of radius $a^{-1 / 2}$ leads to the elliptic operator

$$
\mathcal{L}_{a}=\mathcal{H}_{a}-a \Delta \equiv-\left(\partial_{y}^{2}+a y \partial_{y}+2 a\right)-a \Delta,
$$

where $\Delta$ is the Laplacian of $\mathbb{S}^{1}$. The operator $-\mathcal{H}_{a}=\partial_{y}^{2}+a y \partial_{y}+2 a$ is commonly seen in geometric evolution equations. It is self-adjoint in the weighted Hilbert space $L^{2}\left(\mathbb{R}, e^{-a y^{2} / 2} \mathrm{~d} y\right)$ with spectrum $\operatorname{spec}\left(\mathcal{H}_{a}\right)=\{a(j-2)\}_{j \geq 0}$. Its eigenfunctions are the Hermite polynomials $h_{a, j}$; in particular, its (weakly) unstable eigenspaces are spanned by $h_{a, 0}=1, h_{a, 1}=y$, and $h_{a, 2}=y^{2}-a^{-1}$. The spectrum $\left\{k^{2}\right\}_{k \geq 0}$ of $-\Delta$ is also well known, and its lowest nontrivial eigenspace is spanned by the restriction to $\mathbb{S}^{1}$ of the coordinate functions $\left.x_{1}\right|_{\mathbb{S}^{1}}=\cos \theta$ and $\left.x_{2}\right|_{\mathbb{S}^{1}}=\sin \theta$. Hence $\operatorname{spec}\left(\mathcal{L}_{a}\right)=\left\{a(j-2)+a k^{2} ; j \geq 0, k \geq 0\right\}$, and we can summarize the (weakly) 
unstable eigenvalues of $\mathcal{L}_{a}$ and their associated eigenfunctions in this table:

$\begin{array}{cccc}\text { Eigenvalue } & \text { Multiplicity } & \text { Eigenfunction(s) } & \text { Geometric meaning } \\ -2 a & 1 & 1 & \text { rescale cylinder } \\ -a & 3 & y=\lambda^{-1} x_{0}, x_{1}, x_{2} & \text { translate center of neck } \\ 0 & 3 & y^{2}-a^{-1} ; y x_{1}, y x_{2} & \text { shape neck; tilt cylinder }\end{array}$

In optimal coordinates, a surface is orthogonal to the span of these functions.

Definition 1. We say a cylindrical coordinate system $(x, r, \theta)$ is an optimal system at time $\tau_{1}$ with respect to positive parameters $\lambda_{\mathrm{opt}} \equiv \lambda_{\mathrm{opt}}\left(\tau_{1}\right)$ and $b_{\mathrm{opt}} \equiv b_{\mathrm{opt}}\left(\tau_{1}\right)$ if

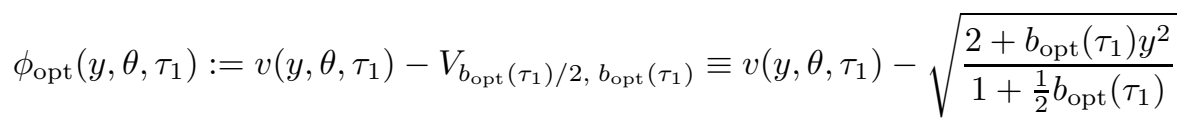

satisfies the orthogonality condition

$$
\phi_{\mathrm{opt}} \perp\left\{1, y, y^{2}-a_{1}^{-1}, \cos \theta, \sin \theta, y \cos \theta, y \sin \theta\right\}
$$

in $L^{2}\left(\mathbb{R} \times \mathbb{S}^{1}, \mathrm{~d} \theta e^{-a_{1} y^{2} / 2} \mathrm{~d} y\right)$, where $v=\lambda_{\mathrm{opt}}^{-1} u, y=\lambda_{\mathrm{opt}}^{-1} x$, and $a_{1}:=\frac{1}{2}-\frac{1}{4} b_{\mathrm{opt}}$.

Having constructed optimal coordinates at time $\tau_{1}$, we then observe that by a straightforward extension of the implicit function theorem argument developed in [14], there exists a (possibly small) earlier time interval $\left[\tau_{0}, \tau_{1}\right]$ and $C^{1}$ functions $\lambda(\tau), a(\tau), b(\tau)$, and $\beta_{0}(\tau), \ldots, \beta_{4}(\tau)$ defined for $\tau \in\left[\tau_{0}, \tau_{1}\right]$, such that the quantity

$$
\tilde{\phi}(y, \theta, \tau):=v(y, \theta, \tau)-V_{3 / 2-2 a(\tau), b(\tau)} \equiv v(y, \theta, \tau)-\sqrt{\frac{2+b(\tau) y^{2}}{2-2 a(\tau)}}
$$

can be parameterized in the form

$$
\tilde{\phi}(y, \theta, \tau)=\beta_{0} y+\beta_{1} \cos \theta+\beta_{2} \sin \theta+\beta_{3} y \cos \theta+\beta_{4} y \sin \theta+\phi(y, \theta, \tau),
$$

where the orthogonality conditions

$$
\phi \perp\left\{1, y, y^{2}-a^{-1}, \cos \theta, \sin \theta, y \cos \theta, y \sin \theta\right\}
$$

hold in $L^{2}\left(\mathbb{R} \times \mathbb{S}^{1}, \mathrm{~d} \theta e^{-a(\tau) y^{2} / 2} \mathrm{~d} y\right)$ for $\tau \in\left[\tau_{0}, \tau_{1}\right]$, with the boundary conditions $\lambda\left(\tau_{1}\right)=\lambda_{\text {opt }}\left(\tau_{1}\right), a\left(\tau_{1}\right)=a_{1}$ (recall that $\left.a=-\lambda \partial_{t} \lambda\right)$, and $b\left(\tau_{1}\right)=b_{\text {opt }}\left(\tau_{1}\right)$, and with the new "translate" and "tilt" parameters satisfying the boundary conditions

$$
\beta_{0}\left(\tau_{1}\right)=\beta_{1}\left(\tau_{1}\right)=\beta_{2}\left(\tau_{1}\right)=\beta_{3}\left(\tau_{1}\right)=\beta_{4}\left(\tau_{1}\right)=0 .
$$

The next step of the argument, accomplished in the second bootstrap machine, is to obtain sufficiently good control on the parameters $\beta_{0}, \ldots, \beta_{4}$ to conclude that the sequence of optimal coordinate systems constructed at discrete times $\tau\left(t_{1}\right)<$ $\tau\left(t_{2}\right)<\cdots<T$ converges. We accomplish this by proving that the difference between an optimal system at time $t_{1}$ and an optimal system at a later time $t_{2}$ is of order $b^{2}\left(t_{1}\right)$, where $b(t)=\{1+o(1)\}(-\log (T-t))^{-1} \rightarrow 0$ as $t \nearrow T$.

This concludes our heuristic outline of the proof. We now provide the details needed to make everything rigorous. 


\section{How THE SOLUtion EVOLVES}

Given integers $m, n \geq 0$ and a real number $k \geq 0$, we define

$$
v_{m, n, k}:=v^{-k}\left(\partial_{y}^{m} \partial_{\theta}^{n} v\right)
$$

The following result is an immediate consequence of Lemma 3.1 and Corollary 3.3 from our earlier work in [13]:

Lemma 1. The quantity $v_{m, n, k}^{2}$ evolves by

$$
\begin{aligned}
\partial_{\tau}\left(v_{m, n, k}^{2}\right)= & A_{v}\left(v_{m, n, k}^{2}\right)+2\left[(k+1) v^{-2}-(m+k-1) a\right] v_{m, n, k}^{2} \\
& -B_{m, n, k}+2 E_{m, n, k} v_{m, n, k},
\end{aligned}
$$

where $B_{m, n, k}:=A_{v}\left(v_{m, n, k}^{2}\right)-2 v_{m, n, k} A_{v} v_{m, n, k}$ satisfies the inequality

$$
B_{m, n, k} \geq 2 \frac{\left(\partial_{y} v_{m, n, k}\right)^{2}+\left(v^{-1} \partial_{\theta} v_{m, n, k}\right)^{2}}{1+p^{2}+q^{2}} \geq 0,
$$

and where the commutator terms $E_{m, n, k}=\sum_{\ell=0}^{5} E_{m, n, k, \ell}$ are given by

$$
\begin{aligned}
& E_{m, n, k, 0}:=-k v^{-1} v_{m, n, k}\left(A_{v} v+a y \partial_{y} v\right) \\
& E_{m, n, k, 1}:=v^{-k} \partial_{y}^{m} \partial_{\theta}^{n}\left(F_{1} \partial_{y}^{2} v\right)-F_{1} \partial_{y}^{2} v_{m, n, k} \\
& E_{m, n, k, 2}:=v^{-k} \partial_{y}^{m} \partial_{\theta}^{n}\left(v^{-2} F_{2} \partial_{\theta}^{2} v\right)-v^{-2} F_{2} \partial_{\theta}^{2} v_{m, n, k}, \\
& E_{m, n, k, 3}:=v^{-k} \partial_{y}^{m} \partial_{\theta}^{n}\left(v^{-1} F_{3} \partial_{y} \partial_{\theta} v\right)-v^{-1} F_{3} \partial_{y} \partial_{\theta} v_{m, n, k}, \\
& E_{m, n, k, 4}:=v^{-k} \partial_{y}^{m} \partial_{\theta}^{n}\left(v^{-2} F_{4} \partial_{\theta} v\right)-v^{-2} F_{4} \partial_{\theta} v_{m, n, k}, \\
& E_{m, n, k, 5}:=-v^{-k} \partial_{y}^{m} \partial_{\theta}^{n}\left(v^{-1}\right)-v^{-2} v_{m, n, k} .
\end{aligned}
$$

The utility of estimate (3.3) is that once one has suitable first-order estimates for $v$, one can bound $1+p^{2}+q^{2}$ from above, whereupon $B_{m, n, k}$ contributes useful higherorder terms of the form $-\varepsilon\left(v_{m+1, n, k}^{2}+v_{m, n+1, k+1}^{2}\right)$ to the evolution equation (3.2) satisfied by $v_{m, n, k}^{2}$.

In the bootstrapping arguments made in this paper, we must estimate the nonlinear "error terms" $E_{m, n, k, \ell}$ defined in display (3.4). In Section 3 of [13], we made the following observations, which we freely use here in estimating these quantities.

Remark 1. For any $i, j \geq 0$ and $\ell=1, \ldots, 4$, there exist constants $C_{i, j, \ell}$ such that

$$
\left|\partial_{p}^{i} \partial_{q}^{j} F_{\ell}(p, q)\right| \leq C_{i, j, \ell}
$$

for all $p, q \in \mathbb{R}$. Moreover, $E_{m, n, k, 0}=0$ if $k=0$, and $E_{m, n, k, 5}=0$ if $m+n=1$.

\section{The First Bootstrap Machine}

4.1. Inputs. We now present the inputs to our first bootstrap machine, whose structure we describe below. By standard regularity theory for quasilinear parabolic equations, if the initial data satisfy the Main Assumptions in Section 2.2 for 
sufficiently small $b_{0}$ and $c_{0}$, then solutions originating from such data will satisfy the properties below for a sufficiently short time interval $\left[0, \tau_{1}\right]^{6}$

Most of these properties are global, while others are local in nature. In many of the arguments that follow, we separately treat the inner region $\left\{\beta y^{2} \leq 20\right\}$ and the outer region $\left\{\beta y^{2} \geq 20\right\}$, where $\beta(\tau):=\left(\kappa_{0}+\tau\right)^{-1}$. Note that the inner region here corresponds to the union of the parabolic and intermediate regions in [3]. Note also that our Main Assumptions imply that slightly stronger conditions hold for the inner region than for the outer. This is natural, because it is in the inner region that one expects the solution $v$ of (2.3) to be closest to the formal solution $V_{1 / 2, \beta}$ of the equation $\frac{1}{2} y \partial_{y} V-\frac{1}{2} V+V^{-1}=0$ that is the adiabatic approximation of (2.3).

To state the global conditions, we decompose the solution into $\theta$-independent and $\theta$-dependent parts $v_{1}, v_{2}$, respectively, defined by

$$
v_{1}(y, \tau):=\frac{1}{2 \pi} \int_{0}^{2 \pi} v(y, \theta, \tau) \mathrm{d} \theta \quad \text { and } \quad v_{2}(y, \theta, \tau):=v(y, \theta, \tau)-v_{1}(y, \tau) .
$$

Here are the global conditions:

[C0] For $\tau \in\left[0, \tau_{1}\right]$, the solution has a uniform lower bound $v(\cdot, \cdot, \tau) \geq \kappa_{0}^{-1}$.

[C1] For $\tau \in\left[0, \tau_{1}\right]$, the solution satisfies the first-order estimates

$$
\left|\partial_{y} v\right| \lesssim \beta^{\frac{2}{5}} v^{\frac{1}{2}}, \quad\left|\partial_{\theta} v\right| \lesssim \beta^{\frac{3}{2}} v^{2}, \quad \text { and } \quad\left|\partial_{\theta} v\right| \lesssim v .
$$

$[\mathrm{C} 2]$ For $\tau \in\left[0, \tau_{1}\right]$, the solution satisfies the second-order estimates

$$
\left|\partial_{y}^{2} v\right| \lesssim \beta^{\frac{3}{5}}, \quad\left|\partial_{y} \partial_{\theta} v\right| \lesssim \beta^{\frac{3}{2}} v, \quad\left|\partial_{y} \partial_{\theta} v\right| \lesssim 1, \quad \text { and } \quad\left|\partial_{\theta}^{2} v\right| \lesssim \beta^{\frac{3}{2}} v^{2} .
$$

[C3] For $\tau \in\left[0, \tau_{1}\right]$ the solution satisfies the third-order decay estimates

$$
\left|\partial_{y}^{3} v\right| \lesssim \beta \text { and } v^{-n}\left|\partial_{y}^{m} \partial_{\theta}^{n} v\right| \lesssim \beta^{\frac{3}{2}}
$$

for $m+n=3$ with $n \geq 1$, as well as a "smallness estimate" that

$$
\beta^{-\frac{11}{20}}\left(\left|\partial_{y}^{3} v\right|+\left|\partial_{y}^{2} \partial_{\theta} v\right|\right)+\left|\partial_{y} \partial_{\theta}^{2} v\right|+v^{-1}\left|\partial_{\theta}^{3} v\right| \lesssim\left(\beta_{0}+\varepsilon_{0}\right)^{\frac{1}{40}}
$$

holds for some $\varepsilon_{0}=\varepsilon_{0}\left(b_{0}, c_{0}\right) \ll 1 .^{7}$

[Ca] For $\tau \in\left[0, \tau_{1}\right]$, the parameter $a$ satisfies

$$
\frac{1}{2}-\kappa_{0}^{-1} \leq a \leq \frac{1}{2}+\kappa_{0}^{-1}
$$

[Cg] For $\tau \in\left[0, \tau_{1}\right]$, one has $\langle y\rangle^{-1}\left|\partial_{y} v\right| \leq M^{\frac{1}{4}} \beta$, where $M \gg 1$ is the constant introduced in Section 2.1.

[Cr] There exists $0<\delta \ll 1$ such that the scale-invariant bound $\left|v_{2}\right| \leq \delta v_{1}$ holds everywhere for $\tau \in\left[0, \tau_{1}\right]$.

$[\mathrm{Cs}]$ For $\tau \in\left[0, \tau_{1}\right]$, one has Sobolev bounds $\left\|v^{-n} \partial_{y}^{m} \partial_{\theta}^{n} v\right\|_{\mu}<\infty$ whenever $4 \leq m+n \leq 7$

[Ct] For $\tau \in\left[0, \tau_{1}\right]$, the solution is "tame at infinity" in the sense that for $4 \leq m+n \leq 7$, one has $\left\|v^{-n} \partial_{y}^{m} \partial_{\theta}^{n} v\right\|_{L^{\infty}}<\infty$.

\footnotetext{
${ }^{6}$ In order to obtain some of the derivative bounds here, we use a general interpolation result, Lemma B.2 from [13]. Note that Lemmas B.1 and B.2 in [13] use only the $2 \pi$-periodicity of $v(\cdot, \theta, \cdot)$, hence apply here as well.

${ }^{7}$ The smallness estimate is only used in the proof of Theorem 4.
} 
Note that the global gradient Condition $[\mathrm{Cg}]$ posits the $\beta$ decay rate of the formal solution $V(y, \tau)=\sqrt{2+\beta y^{2}}$, but with a large constant $M^{\frac{1}{4}} \gg 1$. The effect of our bootstrap argument will be to sharpen that constant. Remark 3 (below) shows that Condition [Cb] in Section 6, which is directly implied by our Main Assumptions in Section 2.2 , in turn implies that the global gradient bound $[\mathrm{Cg}]$ holds, along with extra properties that are local to the inner region.

The extra conditions for the inner region are as follows.

[C0i] For $\tau \in\left[0, \tau_{1}\right]$ and $\beta y^{2} \leq 20$, the quantity $v$ is uniformly bounded from above and below: there exists $C_{*}$ such that

$$
\frac{19}{20} \sqrt{2} \equiv g(y, \beta) \leq v(y, \cdot, \cdot) \leq C_{*} .
$$

[C1i] For $\tau \in\left[0, \tau_{1}\right]$ and $\beta y^{2} \leq 20$, the solution satisfies the stronger first-order estimates

$$
\left|\partial_{y} v\right| \lesssim \beta^{\frac{1}{2}} v^{\frac{1}{2}} \text { and }\left|\partial_{\theta} v\right| \lesssim \kappa_{0}^{-\frac{1}{2}} v .
$$

[C2i] For $\tau \in\left[0, \tau_{1}\right]$ and $\beta y^{2} \leq 20$, the $\theta$-dependent part $^{8}$ of the solution satisfies

$$
\left|v_{ \pm}\right|+\left|\left(\partial_{y} v\right)_{ \pm}\right| \lesssim \beta^{2}
$$

The only differences above from [13] are Condition [Ct], which follows from the new Assumption [A7], and [C2i], which follows from the revised [A1]. As explained in Section 2.3 and Remark 2 (below), $[\mathrm{Ct}]$ and [C2i] allow us to compensate for the discrete symmetries assumed in [13] that may not hold here. We make the consequences of these changes clear in the proofs that follow.

4.2. Outputs. The output of this machine consist of the following estimates, which collectively improve Conditions $[\mathrm{C} 0]-[\mathrm{C} 3],[\mathrm{Cs}],[\mathrm{Cr}],[\mathrm{Cg}]$, and $[\mathrm{CO}]-[\mathrm{C} 1 \mathrm{i}]$ :

$$
\begin{gathered}
v(y, \theta, \tau) \geq g(y, \beta), \quad v=\mathcal{O}(\langle y\rangle) \text { as }|y| \rightarrow \infty ; \\
v^{-\frac{1}{2}}\left|\partial_{y} v\right| \lesssim \beta^{\frac{1}{2}}, \quad\left|\partial_{y} v\right| \leq \varepsilon_{0}, \quad v^{-2}\left|\partial_{\theta} v\right| \lesssim \beta^{\frac{33}{20}}, \quad v^{-1}\left|\partial_{\theta} v\right| \lesssim \kappa_{0}^{-\frac{1}{2}} \\
\beta\left|\partial_{y}^{2} v\right|+v^{-1}\left|\partial_{y} \partial_{\theta} v\right|+v^{-2}\left|\partial_{\theta}^{2} v\right| \lesssim \beta^{\frac{33}{20}} \\
\left|\partial_{y} \partial_{\theta} v\right|+v^{-1}\left|\partial_{\theta}^{2} v\right| \lesssim\left(\beta_{0}+\varepsilon_{0}\right)^{\frac{1}{20}} \\
\beta^{\frac{1}{2}}\left|\partial_{y}^{3} v\right|+v^{-1}\left|\partial_{y}^{2} \partial_{\theta} v\right|+v^{-2}\left|\partial_{y} \partial_{\theta}^{2} v\right|+v^{-3}\left|\partial_{\theta}^{3} v\right| \lesssim \beta^{\frac{33}{20}} \\
\beta^{-\frac{11}{20}}\left(\left|\partial_{y}^{3} v\right|+\left|\partial_{y}^{2} \partial_{\theta} v\right|\right)+\left|\partial_{y} \partial_{\theta}^{2} v\right|+v^{-1}\left|\partial_{\theta}^{3} v\right| \lesssim\left(\beta_{0}+\varepsilon_{0}\right)^{\frac{1}{20}}
\end{gathered}
$$

Here, $\beta_{0} \equiv \beta(0)$ and $\varepsilon_{0}$ are independent of $\tau_{1}$. Therefore, these improvements allow us to propagate the assumptions above forward in time in our proof of the Main Theorem.

\footnotetext{
${ }^{8}$ Recall that $v_{ \pm}$and $\left(\partial_{y} v\right)_{ \pm}$are defined by $(2.9)$
} 
4.3. Structure. In the first step of the bootstrap argument, we derive improved estimates for $v$ and its first derivatives in the outer region.

Theorem 1. Suppose a solution $v=v(y, \theta, \tau)$ of (2.3) satisfies Assumption [A1] at $\tau=0$. If for $\tau \in\left[0, \tau_{1}\right]$, Conditions [Ca] and [CO]-[C2] hold in the outer region $\left\{\beta y^{2} \geq 20\right\}$, and Conditions [C0i]-[C1i] hold on its boundary, then throughout the outer region $\left\{\beta y^{2} \geq 20\right\}$, one has estimates

$$
\begin{gathered}
v(y, \cdot, \cdot) \geq 4, \\
\left|\partial_{y} v\right| \lesssim \beta^{\frac{1}{2}} v^{\frac{1}{2}}, \\
\left|\partial_{y} v\right| \leq \varepsilon_{0}, \\
\left|\partial_{\theta} v\right| \leq C_{0} \kappa_{0}^{-\frac{1}{2}} v,
\end{gathered}
$$

for all $\tau \in\left[0, \tau_{1}\right]$, where $\varepsilon_{0}$ and $C_{0}$ depend only on the initial data and not on $\tau_{1}$.

Proof. Except for estimate (4.4), the statements and their proofs are identical to those stated in Theorem 4.2 and proved in Section 5 of [13]. Indeed, the reader may check that the proofs there used neither the fact that $v(y, \cdot, \cdot)$ was assumed to be an even function of $y$, nor that $v(y, \cdot, \cdot)$ was assumed to be orthogonal to the lowest two eigenspaces $\operatorname{span}\{1, \sin \theta, \cos \theta\} \subset L^{2}\left(\{y\} \times \mathbb{S}^{1}\right)$ of the Laplacian on $\mathbb{S}^{1}$.

We now show how to improve the bound $\left|\partial_{y} v\right| \lesssim 1$ proved in in Section 5 of [13] to the bound $\left|\partial_{y} v\right| \leq \varepsilon_{0}$ needed here. In our earlier work, we showed that $1+\left(\partial_{y} v\right)^{4}$ was bounded above for all time by the solution of the IVP

$$
\begin{aligned}
& \frac{d}{d \tau} \varphi=C \beta^{\frac{6}{5}} \varphi \\
& \varphi(0)=\sup _{\tau=0}\left\{1+\left(\partial_{y} v\right)^{4}\right\} .
\end{aligned}
$$

Noting that

$$
\frac{\lim _{\tau \rightarrow \infty} \varphi(\tau)}{\varphi(0)}=\exp \left(\frac{5 C}{\kappa_{0}^{1 / 5}}\right)
$$

where $C$ does not increase as we increase $\kappa_{0}$, we obtain the result by choosing $\kappa_{0}$ sufficiently large and $b_{0}$, hence $\varphi(0)$, sufficiently close to 1 .

Note that estimate (4.4) provides $\varepsilon_{0}$ such that $\left|\partial_{y} v\right| \leq \varepsilon_{0}$ in the outer region for $\tau \in\left[0, \tau_{1}\right]$. By Condition [C0i], one has $v\left( \pm \sqrt{20} \beta^{-\frac{1}{2}}\right) \leq c^{2}$ for some $c>0$. Hence by quadrature, one obtains the immediate corollary that

$$
v=\mathcal{O}(\langle y\rangle) \text { as }|y| \rightarrow \infty .
$$

In the second step, we derive estimates for second and third derivatives of $v$. In the outer region, these estimates are obtained by maximum principle arguments, exactly as in [13], using the noncompact maximum principle derived in Appendix C of that paper. In the inner region, we introduce and bound suitable Lyapunov functionals and then employ Sobolev embedding. These arguments require integration by parts. 
Remark 2. For $1 \leq m+n \leq 7$, Condition [Cs] implies that $\left\|v_{m, n, n}\right\|_{L^{\infty}}<\infty$ for $\tau \in\left[0, \tau_{1}\right]$. Using this and (4.6), it is straightforward to verify that we may integrate by parts in y as was done in [13] - without using reflection symmetry. Two special but important examples are the facts that for $0 \leq r+s, m+n \leq 5$, one has

$$
\left\langle\partial_{y}^{2} v_{m, n, n}, v_{r, s, s}\right\rangle_{\mu}=-\left\langle v_{m+1, n, n}, v_{r+1, s, s}+v_{r, s, s} \partial_{y}(\log \mu)\right\rangle_{\mu} ;
$$

and for $1 \leq m+n \leq 5$, one has

$$
\begin{aligned}
-\left\langle y \partial_{y} v_{m, n, n}, v_{m, n, n}\right\rangle_{\mu} & =\frac{1}{2}\left\|v_{m, n, n}\right\|_{\mu}^{2}+\frac{1}{2}\left\langle v_{m, n, n}, y \partial_{y}(\log \mu) v_{m, n, n}\right\rangle_{\mu} \\
& \leq \frac{1}{2}\left\|v_{m, n, n}\right\|_{\mu}^{2},
\end{aligned}
$$

because $y \partial_{y}(\log \mu) \leq 0$.

Theorem 2. Suppose that a solution $v=v(y, \theta, \tau)$ of equation (2.3) satisfies Assumption [A1] at $\tau=0$, and Conditions [Ca], [CO]-[C3], [Cs], [Cr], [Cg], and [C0i]-[C2i] for $\tau \in\left[0, \tau_{1}\right]$. Then for the same time interval, the solution satisfies the following pointwise bounds throughout the inner region $\left\{\beta y^{2} \leq 20\right\}$ :

$$
\beta\left|\partial_{y}^{2} v\right|+v^{-1}\left|\partial_{y} \partial_{\theta} v\right|+v^{-2}\left|\partial_{\theta}^{2} v\right| \lesssim \beta^{\frac{33}{20}}
$$

and

$$
\beta^{\frac{1}{2}}\left|\partial_{y}^{3} v\right|+v^{-1}\left|\partial_{y}^{2} \partial_{\theta} v\right|+v^{-2}\left|\partial_{y} \partial_{\theta}^{2} v\right|+v^{-3}\left|\partial_{\theta}^{3} v\right| \lesssim \beta^{\frac{33}{20}},
$$

The fact that we do not achieve the expected $\beta^{2}$ decay on the RHS of estimates (4.7)-(4.8) is due to our use of Sobolev embedding with respect to the weighted measure for $L_{\mu}^{2}$ introduced in Section 2.1.

We prove Theorem 2 in Section 5 . This task requires us to modify the arguments used in [13]: those arguments use evenness of $v(y, \cdot, \cdot)$ and $\pi$-periodicity of $v(\cdot, \theta, \cdot)$, neither of which are assumed here.

Using Theorem 2 to ensure that they hold on the boundary of the inner region, we then extend its estimates to the outer region.

Theorem 3. Suppose that a solution $v=v(y, \theta, \tau)$ of equation (2.3) satisfies Assumption [A1] at $\tau=0$, and Conditions [Ca] and [CO]-[C3] for $\beta y^{2} \geq 20$ and $\tau \in\left[0, \tau_{1}\right]$. Then the estimates

$$
\beta\left|\partial_{y}^{2} v\right|+v^{-1}\left|\partial_{y} \partial_{\theta} v\right|+v^{-2}\left|\partial_{\theta}^{2} v\right| \lesssim \beta^{\frac{33}{20}}
$$

and

$$
\beta^{\frac{1}{2}}\left|\partial_{y}^{3} v\right|+v^{-1}\left|\partial_{y}^{2} \partial_{\theta} v\right|+v^{-2}\left|\partial_{y} \partial_{\theta}^{2} v\right|+v^{-3}\left|\partial_{\theta}^{3} v\right| \lesssim \beta^{\frac{33}{20}}
$$

hold throughout the outer region $\left\{\beta y^{2} \geq 20\right\}$ for the same time interval, provided that they hold on the boundary $\left\{\beta y^{2}=20\right\}$.

As an easy corollary, we apply general interpolation results (c.f. [13, Lemma B.2]) to get our final first-order estimate claimed in Section 4.2, namely

$$
\begin{aligned}
\frac{\left|\partial_{\theta} v\right|}{v^{2}} \leq \frac{1}{(1-\delta)^{2}} \frac{\left|\partial_{\theta} v_{2}\right|}{v_{1}^{2}} & \leq \frac{C_{1}}{(1-\delta)^{2}} \max _{\theta \in[0,2 \pi]} \frac{\left|\partial_{\theta}^{2} v_{2}\right|}{v_{1}^{2}} \\
& \leq C_{2} \frac{(1+\delta)^{2}}{(1-\delta)^{2}} \max _{\theta \in[0,2 \pi]} \frac{\left|\partial_{\theta}^{2} v\right|}{v^{2}} \leq C_{3} \beta^{\frac{33}{20}} .
\end{aligned}
$$


The proof of Theorem 3 is identical to that of Theorem 4.6 in Sections 7.1-7.2 of [13], where neither evenness in $y$ nor $\pi$-periodicity in $\theta$ are used.

In the final step, we improve the "smallness estimates" in Condition [C3], producing improved bounds for $\left|\partial_{y} \partial_{\theta}^{2} v\right|$ and $v^{-1}\left|\partial_{\theta}^{3} v\right|$ that serve as inputs to the second bootstrap machine.

Theorem 4. Suppose that a solution $v=v(y, \theta, \tau)$ of equation (2.3) satisfies Assumption [A1] at $\tau=0$, and Conditions [Ca], [CO]-[C3], [Cs], [Cr], [Cg], and [C0i]-[C1i] for $\tau \in\left[0, \tau_{1}\right]$. Then for the same time interval, the solution satisfies

$$
\beta^{-\frac{11}{10}}\left[\left(\partial_{y}^{3} v\right)^{2}+\left(\partial_{y}^{2} \partial_{\theta} v\right)^{2}\right]+\left(\partial_{y} \partial_{\theta}^{2} v\right)^{2}+v^{-2}\left(\partial_{\theta}^{3} v\right)^{2} \lesssim\left(\beta_{0}+\varepsilon_{0}\right)^{\frac{1}{10}} .
$$

As an easy corollary, we again apply general interpolation results to get our final second-order estimates claimed in Section 4.2, namely

$$
\left|\partial_{y} \partial_{\theta} v\right|+v^{-1}\left|\partial_{\theta}^{2} v\right| \lesssim\left(\beta_{0}+\varepsilon_{0}\right)^{\frac{1}{20}}
$$

Once Theorems 2 and 3 are established, the proof of Theorem 4 is identical to that of Theorem 4.7 in Section 7.3 of [13].

Collectively, Theorems 1-4 complete the first bootstrap machine.

\section{IMPROVED ESTIMATES FOR THE INNER REGION}

In this section, we prove Theorem 2 .

Given integers $m, n \geq 0$, we define Lyapunov functionals $\Omega_{m, n}=\Omega_{m, n}(\tau)$ by

$$
\Omega_{m, n}:=\left\|v_{m, n, n}\right\|_{\mu}^{2} \equiv \int_{-\infty}^{\infty} \int_{0}^{2 \pi} v^{-2 n}\left(\partial_{y}^{m} \partial_{\theta}^{n} v\right)^{2} \mathrm{~d} \theta \mu \mathrm{d} y .
$$

Note that $v_{m, n, k}$ is defined in equation (3.1), and $\mu=\mu(y)$ is defined in Section 2.1. By Conditions [C2], [C3], and [Cs], these functionals and their $\tau$-derivatives are well defined if $m+n \leq 5$.

We prove the second- and third-order derivative bounds in Theorem 2 in two steps. (I) We bound certain weighted sums of $\Omega_{m, n}$ with $2 \leq m+n \leq 5$. Here, as in [13], it suffices to show that derivatives of orders three through five decay at the same rates as the estimates we derive for those of second order, rather than at the faster rates one would expect from parabolic smoothing. This somewhat reduces the work involved. (II) We apply Sobolev embedding to get pointwise estimates, using the facts that $|y| \lesssim \beta^{-\frac{1}{2}}$ in the inner region, and that $\mu \sim|y|^{-\frac{6}{5}}$ as $|y| \rightarrow \infty$.

Our lack of discrete symmetry assumptions, in contrast to [13], requires us to modify some of the proofs from our earlier work. Here, we employ three kinds of argument: one for functionals with only $y$-derivatives, one for functionals with "many" $y$-derivatives, and one for functionals with "few" $y$-derivatives. These categories are made precise below.

As immediate consequences of the proofs of Lemmas 6.1-6.3 in [13], we find that the following differential inequalities for the second-order functionals hold pointwise:

Lemma 2. There exists $0<C<\infty$ such that:

(I) the quantity $\left(v_{2,0,0}\right)^{2}=\left(\partial_{y}^{2} v\right)^{2}$ satisfies

$$
\begin{aligned}
\frac{1}{2} \partial_{\tau}\left(v_{2,0,0}\right)^{2} \leq & \left(v_{2,0,0}\right) A_{v}\left(v_{2,0,0}\right)+\left(v^{-2}-a\right)\left(v_{2,0,0}\right)^{2} \\
& +C\left(\beta\left|\left(v_{2,0,0}\right)\right|+\beta^{\frac{11}{10}}\left\{\left|\left(v_{3,0,0}\right)\right|+\left|\left(v_{2,1,1}\right)\right|+\left|\left(v_{1,2,2}\right)\right|\right\}\right) ;
\end{aligned}
$$


(II) the quantity $\left(v_{1,1,1}\right)^{2}=v^{-2}\left(\partial_{y} \partial_{\theta} v\right)^{2}$ satisfies

$$
\begin{aligned}
\frac{1}{2} \partial_{\tau}\left(v_{1,1,1}\right)^{2} \leq & \left(v_{1,1,1}\right) A_{v}\left(v_{1,1,1}\right)+\left(2 v^{-2}-a\right)\left(v_{1,1,1}\right)^{2} \\
& +C\left(\beta^{2}\left\{\left|\left(v_{1,1,1}\right)\right|+\left|\left(v_{2,1,1}\right)\right|+\left|\left(v_{1,2,2}\right)\right|+\left|\left(v_{0,3,3}\right)\right|\right\}+\beta^{3}\left|\left(v_{3,0,0}\right)\right|\right) ;
\end{aligned}
$$

(III) the quantity $\left(v_{0,2,2}\right)^{2}=v^{-4}\left(\partial_{\theta}^{2} v\right)^{2}$ satisfies

$$
\begin{aligned}
\frac{1}{2} \partial_{\tau}\left(v_{0,2,2}\right)^{2} \leq & \left(v_{0,2,2}\right) A_{v}\left(v_{0,2,2}\right)+\left(3 v^{-2}-a\right)\left(v_{0,2,2}\right)^{2} \\
& +C\left(\beta^{2}\left\{\left|\left(v_{1,2,2}\right)\right|+\left|\left(v_{0,3,3}\right)\right|\right\}+\beta^{\frac{21}{10}}\left|\left(v_{0,2,2}\right)\right|+\beta^{3}\left|\left(v_{2,1,1}\right)\right|\right) .
\end{aligned}
$$

We also import various pointwise estimates for derivatives of the coefficients $F_{\ell}$ of the quasilinear elliptic operator $A_{v}$ defined in (2.4)-(2.5). They appear as estimates (6.2)-(6.6) in [13], and follow from Theorem 1, Condition [C2], and Conditions $[\mathrm{C} 0 \mathrm{i}]$ and $[\mathrm{C} 1 \mathrm{i}]$ for the inner region. We state them here as:

Lemma 3. First-order derivatives of the coefficients $F_{\ell}$ obey the bounds

$$
\left|\partial_{y} F_{\ell}\right| \lesssim \beta^{\frac{3}{5}} \quad \text { and } \quad v^{-1}\left|\partial_{\theta} F_{\ell}\right| \lesssim \beta^{\frac{3}{2}}
$$

Second-order derivatives of the coefficients $F_{\ell}$ obey the bounds

$$
\begin{aligned}
\left|\partial_{y}^{2} F_{\ell}\right| & \lesssim \beta^{\frac{6}{5}}+\left|\left(v_{3,0,0}\right)\right|+\left|\left(v_{2,1,1}\right)\right|, \\
v^{-1}\left|\partial_{y} \partial_{\theta} F_{\ell}\right| & \lesssim \beta^{2}+\left|\left(v_{2,1,1}\right)\right|+\left|\left(v_{1,2,2}\right)\right|, \\
v^{-2}\left|\partial_{\theta}^{2} F_{\ell}\right| & \lesssim \beta^{3}+\left|\left(v_{1,2,2}\right)\right|+\left|v_{3,3,0}\right| .
\end{aligned}
$$

In practice, we use these estimates in the following forms, that follow immediately from combining Lemma 3 with Conditions [C1]-[C3], along with the fact that $\left\|\mu^{-1} \partial_{y}^{2} \mu\right\|_{L^{\infty}}=\mathcal{O}\left(M^{-1}\right)$ as $M \rightarrow \infty$. The statement is:

Lemma 4. One has the pointwise estimates

$$
\begin{aligned}
\mu^{-1}\left|\partial_{y}^{2}\left(\mu F_{1}\right)\right| & \lesssim\left|\left(v_{3,0,0}\right)\right|+\left|\left(v_{2,1,1}\right)\right|+\beta^{\frac{6}{5}}+M^{-\frac{1}{2}} \beta^{\frac{3}{5}}+M^{-1} \lesssim \beta+M^{-1}, \\
\left|\partial_{\theta}^{2}\left(v^{-2} F_{2}\right)\right| & \lesssim\left|v_{3,3,0}\right|+\left|\left(v_{1,2,2}\right)\right|+\beta^{\frac{3}{2}} \lesssim \beta^{\frac{3}{2}} \\
\mu^{-1}\left|\partial_{y} \partial_{\theta}\left(v^{-1} \mu F_{3}\right)\right| & \lesssim\left|\left(v_{2,1,1}\right)\right|+\left|\left(v_{1,2,2}\right)\right|+\left(1+M^{-\frac{1}{2}}\right) \beta^{\frac{3}{2}} \lesssim\left(1+M^{-\frac{1}{2}}\right) \beta^{\frac{3}{2}}, \\
\left|\partial_{\theta}\left(v^{-2} F_{4}\right)\right| & \lesssim \beta^{\frac{3}{2}} .
\end{aligned}
$$

5.1. Estimating the time evolution of the Lyapunov functionals. In this section, we prove estimates for the evolution equations satisfied by $\Omega_{m, n}$. The proofs and results are similar but not identical to those in Lemmas 6.5-6.9 and 6.11-6.12 of [13]. The arguments here come in three flavors: one for the case that there are no $\theta$ derivatives $\left(\Omega_{m, 0}\right.$ with $\left.2 \leq m \leq 5\right)$, another for the case that there are "many" $\theta$ derivatives $\left(\Omega_{m, n}\right.$ with $m \in\{0,1\}$ and $\left.m+n \in\{3,4,5\}\right)$, and a third for the remaining cases $\left(\left(\Omega_{m, n}\right.\right.$ with $\left.(m, n) \in\{(2,1),(3,1),(2,2),(4,1),(3,2),(2,3)\}\right)$. 
Lemma 5. There exist constants $0<\varepsilon<C<\infty$ and $\rho=\rho(M)>0$, with $\rho(M) \searrow 0$ as $M \rightarrow \infty$, such that

$$
\begin{aligned}
\frac{d}{d \tau} \Omega_{2,0} \leq-\varepsilon\left(\Omega_{2,0}+\Omega_{3,0}+\Omega_{2,1}\right)+\rho\left\{\beta \Omega_{2,0}^{\frac{1}{2}}+\beta^{\frac{1}{2}} \Omega_{3,0}^{\frac{1}{2}}+\beta^{\frac{11}{10}}\left(\Omega_{2,1}^{\frac{1}{2}}+\Omega_{1,2}^{\frac{1}{2}}\right)\right\} \\
\frac{d}{d \tau} \Omega_{3,0} \leq-\varepsilon\left(\Omega_{3,0}+\Omega_{4,0}+\Omega_{3,1}\right)+\rho \beta^{\frac{3}{2}}\left\{\Omega_{3,0}^{\frac{1}{2}}+\beta^{\frac{1}{10}} \Omega_{4,0}^{\frac{1}{2}}+\Omega_{3,1}^{\frac{1}{2}}+\Omega_{2,2}^{\frac{1}{2}}\right\} \\
+C \beta\left\{\Omega_{3,0}+\Omega_{2,1}+\Omega_{1,2}+\Omega_{0,3}\right\}+C \beta^{\frac{1}{2}} \Omega_{3,0}^{\frac{1}{2}} \Omega_{2,0}^{\frac{1}{2}}, \\
\frac{d}{d \tau} \Omega_{4,0} \leq-\varepsilon\left(\Omega_{4,0}+\Omega_{5,0}+\Omega_{4,1}\right)+C \beta^{\frac{1}{2}}\left(\sum_{4 \leq i+j \leq 5} \Omega_{i, j}\right)+C \beta^{\frac{8}{5}}\left(\sum_{4 \leq i+j \leq 5} \Omega_{i, j}^{\frac{1}{2}}\right), \\
\frac{d}{d \tau} \Omega_{5,0} \leq-\varepsilon\left(\Omega_{5,0}+\Omega_{6,0}+\Omega_{5,1}\right)+C \beta^{\frac{1}{2}}\left(\sum_{5 \leq i+j \leq 6} \Omega_{i, j}\right)+C \beta^{\frac{21}{10}}\left(\sum_{5 \leq i+j \leq 6} \Omega_{i, j}^{\frac{1}{2}}\right) .
\end{aligned}
$$

Proof. We provide a detailed argument for $\Omega_{2,0}$, and sketch the remaining cases.

Argument For $\Omega_{2,0}$ : Applying Lemma 2 and Cauchy-Schwarz in $L_{\mu}^{2}$, we get

$$
\begin{aligned}
\frac{1}{2} \frac{d}{d \tau} \Omega_{2,0} \leq & \left\langle\left(v_{2,0,0}\right), A_{v}\left(v_{2,0,0}\right)\right\rangle_{\mu}+\left\langle\left(v^{-2}-a\right)\left(v_{2,0,0}\right),\left(v_{2,0,0}\right)\right\rangle_{\mu} \\
& +\rho\left\{\beta \Omega_{2,0}^{\frac{1}{2}}+\beta^{\frac{11}{10}}\left(\Omega_{3,0}^{\frac{1}{2}}+\Omega_{2,1}^{\frac{1}{2}}+\Omega_{1,2}^{\frac{1}{2}}\right)\right\} .
\end{aligned}
$$

Define $I_{1}:=\left\langle\left(v_{2,0,0}\right), A_{v}\left(v_{2,0,0}\right)\right\rangle_{\mu}$ and $I_{2}:=\left\langle\left(v^{-2}-a\right)\left(v_{2,0,0}\right),\left(v_{2,0,0}\right)\right\rangle_{\mu}$. Here $\rho$ is a constant that can be made as small as desired, because

$$
\rho=\int_{-\infty}^{\infty} \int_{0}^{2 \pi} \mathrm{d} \theta \mu(y) \mathrm{d} y=2 \pi \int_{-\infty}^{\infty}\left(M+y^{2}\right)^{-\frac{3}{5}} \mathrm{~d} y
$$

which tends to zero as $M \nearrow \infty .^{9}$

By Remark 2, we may integrate $I_{2}$ by parts in $y$, obtaining

$$
\begin{aligned}
I_{2} & =-a \Omega_{2,0}+\left\langle\left(v_{2,0,2}\right),\left(v_{2,0,0}\right)\right\rangle_{\mu} \\
& \leq-a \Omega_{2,0}+\left\langle\left(v_{1,0,2}\right), 2\left(v_{2,0,0}\right)\left(v_{1,0,1}\right)-\left(v_{3,0,0}\right)-\left(v_{2,0,0}\right) \partial_{y}(\log \mu)\right\rangle_{\mu} \\
& \leq-a \Omega_{2,0}+\rho\left(\beta \Omega_{2,0}^{\frac{1}{2}}+\beta^{\frac{1}{2}} \Omega_{3,0}^{\frac{1}{2}}\right) .
\end{aligned}
$$

In the last step, we used the facts that $\left\|\partial_{y} \log \mu\right\|_{L^{\infty}}=\mathcal{O}\left(M^{-\frac{1}{2}}\right)$ as $M \rightarrow \infty$ and that $\partial_{y}(\log \mu)=\mathcal{O}\left(|y|^{-1}\right)$ as $|y| \rightarrow \infty$, along with Condition $[\mathrm{Cg}]$ and estimate (4.3).

Again using Remark 2 and integrating by parts, we find that

$$
I_{1} \leq I_{*}+\frac{a}{2} \Omega_{2,0}
$$

\footnotetext{
${ }^{9}$ Recall that $\mu(y):=\left(M+y^{2}\right)^{-\frac{3}{5}}$, where $M$ is an arbitrarily large constant.
} 
where $I_{*}:=\left\langle\left(v_{2,0,0}\right), F_{1}\left(v_{4,0,0}\right)+F_{2}\left(v_{2,2,2}\right)+F_{3}\left(v_{3,1,1}\right)+F_{4}\left(v_{2,1,2}\right)\right\rangle_{\mu}$. Then defining $F_{0}:=\left(1+p^{2}+q^{2}\right)^{-1}$ lets us estimate

$$
\begin{aligned}
I_{*} \leq & -\left\langle F_{0},\left(v_{3,0,0}\right)^{2}+\left(v_{2,1,1}\right)^{2}\right\rangle_{\mu} \\
& +\frac{1}{2}\left\langle\left(v_{2,0,0}\right)^{2}, \mu^{-1} \partial_{y}^{2}\left(\mu F_{1}\right)+\partial_{\theta}^{2}\left(v^{-2} F_{2}\right)+\mu^{-1} \partial_{y} \partial_{\theta}\left(v^{-1} \mu F_{3}\right)-\partial_{\theta}\left(v^{-2} F_{4}\right)\right\rangle_{\mu} \\
\leq & -(1-\varepsilon)\left(\Omega_{3,0}+\Omega_{2,1}\right)+\varepsilon_{1} \Omega_{2,0} .
\end{aligned}
$$

The first inequality results from applying Cauchy-Schwarz pointwise to the coefficients of the elliptic operator $A_{v}$ after integrating by parts. To get the second inequality, we use estimates (4.4) and (4.5) from Theorem 1 to see that we can bound $F_{0} \geq(1-\varepsilon)$. We use Lemma 4 to control the remaining terms that came from integrating by parts, noting that by taking $\kappa_{0}$ and $M$ sufficiently large, we can by Condition [Ca] ensure that $\varepsilon_{1} \in\left(0, \frac{a}{2}-\varepsilon\right)$. The result follows.

ARGUMENTS FOR THE REMAINING $\Omega_{m, 0}$ : The proofs here are essentially identical to the corresponding cases considered in Lemmas $6.9,6.11$, and 6.12 of [13]. It is important to note that we can use the results of Appendix D from [13] without modification: because we did not need sharp estimates for derivatives of orders three and higher there (as is also true here) we did not need to exploit the discrete symmetry assumptions of that paper to get better results when integrating by parts in $\theta$. As a consequence, the conclusions of Lemmas D.1-D.7, which allow us to estimate the nonlinear terms in the $\Omega_{m, n}$ evolution equations, hold here without modification. For the linear terms, the key estimate, which holds by Conditions [Ca] and $[\mathrm{CO} \mathrm{i}]$ and Theorem 1 when $3 \leq m \leq 5$, is

$$
I_{2}:=\left\langle\left(v^{-2}-(m-1) a\right)\left(v_{m, 0,0}\right),\left(v_{m, 0,0}\right)\right\rangle_{\mu} \leq-\frac{1}{4} \Omega_{m, 0} .
$$

We omit further details.

Lemma 6. There exist $0<\varepsilon<C<\infty$ such that for $(m, n)=(2,1)$, one has

$$
\begin{aligned}
\frac{d}{d \tau} \Omega_{2,1} \leq & -\varepsilon\left(\Omega_{2,1}+\Omega_{3,1}+\Omega_{2,2}\right)+C \beta^{\frac{2}{5}}\left(\Omega_{2,1}+\Omega_{1,2}+\Omega_{0,3}\right) \\
& +C \beta^{2}\left\{\Omega_{2,1}^{\frac{1}{2}}+\beta \Omega_{4,0}^{\frac{1}{2}}+\Omega_{3,1}^{\frac{1}{2}}+\Omega_{2,2}^{\frac{1}{2}}+\Omega_{1,3}^{\frac{1}{2}}+\Omega_{0,4}^{\frac{1}{2}}\right\} ;
\end{aligned}
$$

and for $(m, n) \in\{(3,1),(2,2)\}$, one has

$$
\begin{aligned}
\frac{d}{d \tau} \Omega_{m, n} \leq & -\varepsilon\left(\Omega_{m, n}+\Omega_{m+1, n}+\Omega_{m, n+1}\right)+C \beta^{\frac{1}{2}}\left(\sum_{4 \leq i+j \leq 5} \Omega_{i, j}\right) \\
& +C \beta^{2}\left(\sum_{4 \leq i+j \leq 5} \Omega_{i, j}^{\frac{1}{2}}\right) ;
\end{aligned}
$$

and for $(m, n) \in\{(4,1),(3,2),(2,3)\}$, one has

$$
\begin{aligned}
\frac{d}{d \tau} \Omega_{m, n} \leq & -\varepsilon\left(\Omega_{m, n}+\Omega_{m+1, n}+\Omega_{m, n+1}\right)+C \beta^{\frac{1}{2}}\left(\sum_{5 \leq i+j \leq 6} \Omega_{i, j}\right) \\
& +C \beta^{\frac{21}{10}}\left(\sum_{5 \leq i+j \leq 6} \Omega_{i, j}^{\frac{1}{2}}\right) .
\end{aligned}
$$


Proof. We provide a detailed argument for $\Omega_{2,1}$, and outline the remaining cases. Argument For $\Omega_{2,1}$ : One has

$$
\frac{1}{2} \frac{d}{d \tau} \Omega_{2,1}=I_{1}+I_{2}+I_{3}
$$

with terms $I_{j}$ that we now define. The first term on the RHS is

$$
I_{1}:=\left\langle\left(v_{2,1,1}\right), A_{v}\left(v_{2,1,1}\right)\right\rangle_{\mu} \leq \frac{a}{2} \Omega_{2,1}+I_{*},
$$

where

$I_{*}:=\left\langle\left(v_{2,1,1}\right), F_{1} \partial_{y}^{2}\left(v_{2,1,1}\right)+F_{2} v^{-2} \partial_{\theta}^{2}\left(v_{2,1,1}\right)+F_{3} v^{-1} \partial_{y} \partial_{\theta}\left(v_{2,1,1}\right)+F_{4} v^{-2} \partial_{\theta}\left(v_{2,1,1}\right)\right\rangle_{\mu}$.

The second term in $\frac{1}{2} \frac{d}{d \tau} \Omega_{2,1}$ is

$$
I_{2}:=\left\langle\left(2 v^{-2}-2 a\right)\left(v_{2,1,1}\right),\left(v_{2,1,1}\right)\right\rangle_{\mu} .
$$

Below, we obtain a good estimate for $I_{1}+I_{2}$. Lemma D.1 of [13], which does not require any symmetry assumptions, implies that the remaining contribution to $\frac{1}{2} \frac{d}{d \tau} \Omega_{2,1}$, which is

$$
I_{3}:=\sum_{\ell=0}^{5}\left\langle\left(v_{2,1,1}\right), E_{2,1,1, \ell}\right\rangle_{\mu},
$$

can be estimated by

$$
\left|I_{3}\right| \leq C \beta^{2}\left\{\Omega_{2,1}^{\frac{1}{2}}+\beta \Omega_{4,0}^{\frac{1}{2}}+\Omega_{3,1}^{\frac{1}{2}}+\Omega_{2,2}^{\frac{1}{2}}+\Omega_{1,3}^{\frac{1}{2}}+\Omega_{0,4}^{\frac{1}{2}}\right\}+C \beta^{\frac{2}{5}}\left(\Omega_{2,1}+\Omega_{1,2}+\Omega_{0,3}\right) .
$$

We now study $I_{*}$. Define $F_{0}:=\left(1+p^{2}+q^{2}\right)^{-1}$, as in the proof of Lemma 5 . Then by estimates (4.4) and (4.5) from Theorem 1, there exists $\varepsilon_{1}>0$ such that $F_{0} \geq\left(1-\varepsilon_{1}\right)$. Let $\gamma \in(0,1)$ be a parameter to be chosen. By integrating $I_{*}$ by parts, applying Cauchy-Schwarz pointwise to the coefficients of the quasilinear elliptic operator $A_{v}$, and using Lemma 4 to bound terms containing derivatives of the coefficients of that operator, we find that there exists $\varepsilon_{2}>0$ such that

$$
\begin{aligned}
I_{*} \leq & -\left\langle F_{0},\left\{\partial_{y}\left(v_{2,1,1}\right)\right\}^{2}+\left\{v^{-1} \partial_{\theta}\left(v_{2,1,1}\right)\right\}^{2}\right\rangle_{\mu}+\varepsilon_{2} \Omega_{2,1} \\
\leq- & \left(1-\varepsilon_{1}\right)\left\{\gamma\left(\left\|\partial_{y}\left(v_{2,1,1}\right)\right\|_{\mu}^{2}+\left\|v^{-1} \partial_{\theta}\left(v_{2,1,1}\right)\right\|_{\mu}^{2}\right)+(1-\gamma)\left\|v^{-1} \partial_{\theta}\left(v_{2,1,1}\right)\right\|_{\mu}^{2}\right\} \\
& +\varepsilon_{2} \Omega_{2,1} .
\end{aligned}
$$

Integrating by parts in $y$ and using Conditions $[\mathrm{C} 1]$ and $[\mathrm{C} 2]$ shows that

$$
\left\|\partial_{y}\left(v_{2,1,1}\right)\right\|_{\mu}^{2} \geq \Omega_{3,1}-C \beta^{\frac{3}{5}} \Omega_{2,1} .
$$

Integrating by parts in $\theta$ again using Conditions [C1] and [C2] shows that

$$
\left\|v^{-1} \partial_{\theta}\left(v_{2,1,1}\right)\right\|_{\mu}^{2} \geq \Omega_{2,2}-C \beta^{\frac{3}{2}} \Omega_{2,1} .
$$

Then using Condition [Cr] along with the fact that $\partial_{y}^{2} \partial_{\theta} v$ is orthogonal to constants in $L^{2}\left(\mathbb{S}^{1}\right)$, we integrate by parts in $\theta$ to get

$$
\begin{aligned}
\left\|v^{-1} \partial_{\theta}\left(v_{2,1,1}\right)\right\|_{\mathbb{S}^{1}}^{2} & \geq\left\|v^{-2}\left(\partial_{y}^{2} \partial_{\theta}^{2} v\right)\right\|_{\mathbb{S}^{1}}^{2}-C \beta^{\frac{3}{2}}\left\|\left(v_{2,1,1}\right)\right\|_{\mathbb{S}^{1}}^{2} \\
& \geq\left(\frac{1-\delta}{1+\delta}\right)^{4}\left\|v^{-2}\left(\partial_{y}^{2} \partial_{\theta} v\right)\right\|_{\mathbb{S}^{1}}^{2}-C \beta^{\frac{3}{2}}\left\|\left(v_{2,1,1}\right)\right\|_{\mathbb{S}^{1}}^{2} .
\end{aligned}
$$

Combining these estimates, we obtain $\varepsilon_{3}, \varepsilon_{4}$ such that

$$
I_{*} \leq-\left(1-\varepsilon_{3}\right)\left\{\gamma\left(\Omega_{3,1}+\Omega_{2,2}\right)+(1-\gamma)\left\langle v^{-2}\left(v_{2,1,1}\right),\left(v_{2,1,1}\right)\right\rangle_{\mu}\right\}+\varepsilon_{4} \Omega_{2,1} .
$$


Hence taking $\gamma$ small and using Conditions [Ca] and [C0i] with Assumption [A2], we obtain $\varepsilon$ such that

$$
\begin{aligned}
I_{1}+I_{2} & \leq\left\langle\left\{(1+\varepsilon) v^{-2}+\varepsilon-\frac{3}{2} a\right\}\left(v_{2,1,1}\right),\left(v_{2,1,1}\right)\right\rangle_{\mu}-\varepsilon\left(\Omega_{3,1}+\Omega_{2,2}\right) \\
& \leq-\varepsilon\left(\Omega_{2,1}+\Omega_{3,1}+\Omega_{2,2}\right) .
\end{aligned}
$$

The result follows.

Arguments For the Remaining $\Omega_{m, n}$ : As we note in the proof of Lemma 5 , the conclusions of Lemmas D.1-D.7 from [13], which allow us to estimate the nonlinear terms in the $\Omega_{m, n}$ evolution equations, hold here without modification. For the linear terms, the key estimates are

$$
\begin{aligned}
I_{1}+I_{2} & \leq\left\langle\left\{\frac{3}{4}-\frac{m}{2}+n\left(v^{-2}-\frac{1}{2}\right)+\varepsilon\right\} v_{m, n, n}, v_{m, n, n}\right\rangle_{\mu}-\varepsilon\left(\Omega_{m+n, n}+\Omega_{m, n+1}\right) \\
& \leq-\varepsilon\left(\Omega_{m, n}+\Omega_{m+1, n}+\Omega_{m, n+1}\right) .
\end{aligned}
$$

Condition $[\mathrm{Ca}]$ implies the first estimate. The bound $v^{-2}<\frac{5}{9}$, which follows from Condition [C0i] in the inner region and from estimate (4.2) in the outer region, implies the second estimate above. This concludes the proof for all remaining cases $(m, n) \in\{(3,1),(2,2),(4,1),(3,2),(2,3)\}$.

Finally, we bound the evolutions of the $\Omega_{m, n}$ with relatively "few" $y$-derivatives, namely $(m, n) \in\{(1,2),(0,3),(1,3),(0,4),(1,4),(0,5)\}$. The arguments to prove these estimates are those that are most changed from our prior work [13].

Lemma 7. There exist $0<\varepsilon<C<\infty$ such that for $(m, n) \in\{(1,2),(0,3)\}$ one has

$$
\begin{aligned}
\frac{d}{d \tau} \Omega_{m, n} \leq & -\varepsilon\left(\Omega_{m, n}+\Omega_{m+1, n}+\Omega_{m, n+1}\right)+C \beta^{\frac{3}{5}}\left(\Omega_{2,1}+\Omega_{1,2}+\Omega_{0,3}\right) \\
& +C \beta^{2}\left\{\Omega_{m, n}^{\frac{1}{2}}+\beta \Omega_{4,0}^{\frac{1}{2}}+\Omega_{3,1}^{\frac{1}{2}}+\Omega_{2,2}^{\frac{1}{2}}+\Omega_{1,3}^{\frac{1}{2}}+\Omega_{0,4}^{\frac{1}{2}}\right\} ;
\end{aligned}
$$

and for $(m, n) \in\{(1,3),(0,4)\}$, one has

$$
\begin{aligned}
\frac{d}{d \tau} \Omega_{m, n} \leq & -\varepsilon\left(\Omega_{m, n}+\Omega_{m+1, n}+\Omega_{m, n+1}\right)+C \beta^{\frac{1}{2}}\left(\sum_{4 \leq i+j \leq 5} \Omega_{i, j}\right) \\
& +C \beta^{2}\left(\sum_{4 \leq i+j \leq 5} \Omega_{i, j}^{\frac{1}{2}}\right) ;
\end{aligned}
$$

and for $(m, n) \in\{(1,4),(0,5)\}$, one has

$$
\begin{aligned}
\frac{d}{d \tau} \Omega_{m, n} \leq & -\varepsilon\left(\Omega_{m, n}+\Omega_{m+1, n}+\Omega_{m, n+1}\right)+C \beta^{\frac{1}{2}}\left(\sum_{5 \leq i+j \leq 6} \Omega_{i, j}\right) \\
& +C \beta^{\frac{21}{10}}\left(\sum_{5 \leq i+j \leq 6} \Omega_{i, j}^{\frac{1}{2}}\right) .
\end{aligned}
$$

Proof. We begin with a detailed argument for $\Omega_{1,2}$.

Argument FOR $\Omega_{1,2}$ : As in Lemma 6 , we write

$$
\frac{1}{2} \frac{d}{d \tau} \Omega_{1,2}=I_{1}+I_{2}+I_{3}
$$


Here, the first term on the RHS is

$$
I_{1}:=\left\langle\left(v_{1,2,2}\right), A_{v}\left(v_{1,2,2}\right)\right\rangle_{\mu} \leq \frac{a}{2} \Omega_{1,2}+I_{*},
$$

where

$I_{*}:=\left\langle\left(v_{1,2,2}\right), F_{1} \partial_{y}^{2}\left(v_{1,2,2}\right)+F_{2} v^{-2} \partial_{\theta}^{2}\left(v_{1,2,2}\right)+F_{3} v^{-1} \partial_{y} \partial_{\theta}\left(v_{1,2,2}\right)+F_{4} v^{-2} \partial_{\theta}\left(v_{1,2,2}\right)\right\rangle_{\mu}$ is analyzed below. The second term in $\frac{1}{2} \frac{d}{d \tau} \Omega_{1,2}$ is

$$
I_{2}:=\left\langle\left(3 v^{-2}-2 a\right)\left(v_{1,2,2}\right),\left(v_{1,2,2}\right)\right\rangle_{\mu} .
$$

We estimate $I_{1}+I_{2}$ below. Lemma D.1 of [13], which does not require any symmetry assumptions, implies that the remaining contribution to $\frac{1}{2} \frac{d}{d \tau} \Omega_{1,2}$, which is the quantity $I_{3}:=\sum_{\ell=0}^{5}\left\langle\left(v_{1,2,2}\right), E_{1,2,2, \ell}\right\rangle_{\mu}$, can be estimated by

$\left|I_{3}\right| \leq C \beta^{2}\left\{\Omega_{1,2}^{\frac{1}{2}}+\beta \Omega_{4,0}^{\frac{1}{2}}+\Omega_{3,1}^{\frac{1}{2}}+\Omega_{2,2}^{\frac{1}{2}}+\Omega_{1,3}^{\frac{1}{2}}+\Omega_{0,4}^{\frac{1}{2}}\right\}+C \beta^{\frac{3}{5}}\left(\Omega_{2,1}+\Omega_{1,2}+\Omega_{0,3}\right)$.

To study $I_{*}$, we again define $F_{0}:=\left(1+p^{2}+q^{2}\right)^{-1}$. Then by estimates (4.4) and (4.5) from Theorem 1 , there exists $\varepsilon_{1}>0$ such that $F_{0} \geq\left(1-\varepsilon_{1}\right)$. Let $\gamma \in(0,1)$ be a parameter to be chosen. By integrating $I_{*}$ by parts, applying Cauchy-Schwarz pointwise to the coefficients of the quasilinear elliptic operator $A_{v}$, and using Lemma 4 to bound terms containing derivatives of the coefficients of that operator, we find that there exists $\varepsilon_{2}>0$ such that

$$
\begin{aligned}
I_{*} \leq & -\left\langle F_{0},\left\{\partial_{y}\left(v_{1,2,2}\right)\right\}^{2}+\left\{v^{-1} \partial_{\theta}\left(v_{1,2,2}\right)\right\}^{2}\right\rangle_{\mu}+\varepsilon_{2} \Omega_{1,2} \\
\leq & -\left(1-\varepsilon_{1}\right) \gamma\left\{\left\|\partial_{y}\left(v_{1,2,2}\right)\right\|_{\mu}^{2}+\left\|v^{-1} \partial_{\theta}\left(v_{1,2,2}\right)\right\|_{\mu}^{2}\right\} \\
& -\left(1-\varepsilon_{1}\right)(1-\gamma) \int_{\beta y^{2} \leq 20}\left\|v^{-1} \partial_{\theta}\left(v_{1,2,2}\right)\right\|_{\mathbb{S}^{1}}^{2} \mu \mathrm{d} y+\varepsilon_{2} \Omega_{1,2} .
\end{aligned}
$$

Conditions [C1] and [C2] let us integrate by parts in $y$ to estimate $\left\|\partial_{y}\left(v_{1,2,2}\right)\right\|_{\mu}^{2} \geq$ $\Omega_{2,2}-C \beta^{\frac{3}{5}} \Omega_{1,2}$, and then integrate by parts in $\theta$ to estimate $\left\|v^{-1} \partial_{\theta}\left(v_{1,2,2}\right)\right\|_{\mu}^{2} \geq$ $\Omega_{1,3}-C \beta^{\frac{3}{2}} \Omega_{1,2}$. Thus we obtain $\varepsilon_{3}$ such that

$$
\begin{aligned}
I_{*} \leq- & \left(1-\varepsilon_{1}\right) \gamma\left(\Omega_{2,2}+\Omega_{1,3}\right)+\varepsilon_{3} \Omega_{1,2} \\
& -\left(1-\varepsilon_{1}\right)(1-\gamma) \int_{\beta y^{2} \leq 20}\left\|v^{-3} \partial_{y} \partial_{\theta}^{3} v\right\|_{\mathbb{S}^{1}}^{2} \mu \mathrm{d} y .
\end{aligned}
$$

We now estimate $I_{1}+I_{2}$. Using Conditions [Ca], [C0i], and estimate (4.2), we observe that $I_{2}:=\left\langle\left(3 v^{-2}-2 a\right)\left(v_{1,2,2}\right),\left(v_{1,2,2}\right)\right\rangle_{\mu}$ may be estimated by ${ }^{10}$

$$
\begin{aligned}
I_{2}=- & \frac{2 a}{3} \Omega_{1,2}+\frac{19}{10} \int_{\beta y^{2} \leq 20}\left\|v^{-3} \partial_{y} \partial_{\theta}^{2} v\right\|_{\mathbb{S}^{1}}^{2} \mu \mathrm{d} y \\
& +\int_{\beta y^{2} \leq 20} \int_{\mathbb{S}^{1}}\left(\frac{11}{10} v^{-2}-\frac{4 a}{3}\right)\left(v_{1,2,2}\right)^{2} \mathrm{~d} \theta \mu \mathrm{d} y \\
& +\int_{\beta y^{2}>20} \int_{\mathbb{S}^{1}}\left(3 v^{-2}-\frac{4 a}{3}\right)\left(v_{1,2,2}\right)^{2} \mathrm{~d} \theta \mu \mathrm{d} y \\
\leq- & \frac{2 a}{3} \Omega_{1,2}+\frac{19}{10} \int_{\beta y^{2} \leq 20}\left\|v^{-3} \partial_{y} \partial_{\theta}^{2} v\right\|_{\mathbb{S}^{1}}^{2} \mu \mathrm{d} y .
\end{aligned}
$$

\footnotetext{
${ }^{10}$ See below for the formula we use to get this decomposition for general $(m, n)$.
} 
Combining this with the estimates above, we obtain

$$
I_{1}+I_{2} \leq\left(\varepsilon_{3}-\frac{a}{6}\right) \Omega_{1,2}-\left(1-\varepsilon_{1}\right) \gamma\left(\Omega_{2,2}+\Omega_{1,3}\right)+\int_{\beta y^{2} \leq 20} \Xi \mu \mathrm{d} y,
$$

where the quantity that remains to be controlled is

$$
\Xi:=\frac{19}{10}\left\|v^{-3} \partial_{y} \partial_{\theta}^{2} v\right\|_{\mathbb{S}^{1}}^{2}-\left(1-\varepsilon_{1}\right)(1-\gamma)\left\|v^{-3} \partial_{y} \partial_{\theta}^{3} v\right\|_{\mathbb{S}^{1}}^{2} .
$$

To estimate $\Xi$, let $\mathbb{P}$ denote orthogonal projection onto $\operatorname{span}\left\{e^{ \pm i \theta}\right\} \subset L^{2}\left(\mathbb{S}^{1}\right)$. Then using Condition [Cr], it is easy to see that there exists $\varepsilon_{4}$ depending only on $\delta$ such that in the inner region,

$$
\left\|v^{-3} \partial_{y} \partial_{\theta}^{3} v\right\|_{\mathbb{S}^{1}}^{2} \geq\left(1-\varepsilon_{4}\right)\left\{\left\|v^{-3} \mathbb{P}\left(\partial_{y} \partial_{\theta}^{3} v\right)\right\|_{\mathbb{S}^{1}}^{2}+\left\|v^{-3}(1-\mathbb{P})\left(\partial_{y} \partial_{\theta}^{3} v\right)\right\|_{\mathbb{S}^{1}}^{2}\right\} .
$$

Again using $[\mathrm{Cr}]$ together with the fact that $(1-\mathbb{P})\left(\partial_{y} \partial_{\theta}^{3} v\right) \perp \operatorname{span}\left\{1, e^{ \pm i \theta}\right\}$, we integrate by parts as in [13] to estimate that

$$
\begin{aligned}
\left\|v^{-3}(1-\mathbb{P})\left(\partial_{y} \partial_{\theta}^{3} v\right)\right\|_{\mathbb{S}^{1}}^{2} & =\left\|v^{-3} \partial_{\theta}\left\{(1-\mathbb{P})\left(\partial_{y} \partial_{\theta}^{2} v\right)\right\}\right\|_{\mathbb{S}^{1}}^{2} \\
& \geq 4\left(1-\varepsilon_{4}\right)\left\|v^{-3}(1-\mathbb{P})\left(\partial_{y} \partial_{\theta}^{2} v\right)\right\|_{\mathbb{S}^{1}}^{2} .
\end{aligned}
$$

Then using the fact that $\partial_{y} \partial_{\theta}^{2} v \perp \operatorname{span}\{1\}$ in $L^{2}\left(\mathbb{S}^{1}\right)$, we obtain

$$
\begin{aligned}
\Xi & \leq\left\{\frac{19}{10}\left(1+\varepsilon_{4}\right)-\left(1-\varepsilon_{4}\right)\left(1-\varepsilon_{1}\right)(1-\gamma)\right\}\left\|v^{-3} \mathbb{P}\left(\partial_{y} \partial_{\theta}^{2} v\right)\right\|_{\mathbb{S}^{1}}^{2} \\
& +\left\{\frac{19}{10}\left(1+\varepsilon_{4}\right)-4\left(1-\varepsilon_{4}\right)\left(1-\varepsilon_{1}\right)(1-\gamma)\right\}\left\|v^{-3}(1-\mathbb{P})\left(\partial_{y} \partial_{\theta}^{2} v\right)\right\|_{\mathbb{S}^{1}}^{2} .
\end{aligned}
$$

It is easy to choose $\gamma \in(0,1)$ so that the second quantity in braces above is negative. To control the remaining term, we observe that the new Condition [C2i] implies that $\left\|\mathbb{P}\left(\partial_{y} \partial_{\theta}^{2} v\right)\right\|_{\mathbb{S}^{1}} \lesssim \beta^{2}$, and then use Cauchy-Schwarz to see that

$$
\int_{\beta y^{2} \leq 20}\left\|v^{-3} \mathbb{P}\left(\partial_{y} \partial_{\theta}^{2} v\right)\right\|_{\mathbb{S}^{1}}^{2} \mu \mathrm{d} y \leq \rho \beta^{2} \Omega_{1,2}^{\frac{1}{2}},
$$

where $\rho(M) \searrow 0$ as $M \rightarrow \infty$. The result follows.

Arguments FOR the Remaining $\Omega_{m, n}$ : The nonlinear terms are estimated as above, mutatis mutandis. To control the linear terms, we first observe that

$$
\begin{aligned}
I_{2}:= & \int_{\mathbb{R}} \int_{\mathbb{S}^{1}}\left\{(n+1) v^{-2}-(m+n-1) a\right\}\left(v_{m, n, n}\right)^{2} \mathrm{~d} \theta \mu \mathrm{d} y \\
=- & \frac{2 a}{3} \Omega_{m, n}+\frac{26+n-9 m}{10} \int_{\beta y^{2} \leq 20}\left\|v^{-(n+1)} \partial_{y}^{m} \partial_{\theta}^{n} v\right\|_{\mathbb{S}^{1}}^{2} \mu \mathrm{d} y \\
& +\int_{\beta y^{2} \leq 20}\left\{\frac{9(m+n)-16}{10} v^{-2}-\left(m+n-\frac{5}{3}\right) a\right\}\left(v_{m, n, n}\right)^{2} \mathrm{~d} \theta \mu \mathrm{d} y \\
& +\int_{\beta y^{2}>20}\left\{(n+1) v^{-2}-\left(m+n-\frac{5}{3}\right) a\right\}\left(v_{m, n, n}\right)^{2} \mathrm{~d} \theta \mu \mathrm{d} y \\
\leq- & \frac{2 a}{3} \Omega_{m, n}+\frac{26+n-9 m}{10} \int_{\beta y^{2} \leq 20}\left\|v^{-(n+1)} \partial_{y}^{m} \partial_{\theta}^{n} v\right\|_{\mathbb{S}^{1}}^{2} \mu \mathrm{d} y .
\end{aligned}
$$

The estimate here again follows from Conditions [Ca], [C0i], and estimate (4.2). Thus we obtain

$$
I_{1}+I_{2} \leq\left(\varepsilon_{3}-\frac{a}{6}\right) \Omega_{m, n}-\left(1-\varepsilon_{1}\right) \gamma\left(\Omega_{m+1, n}+\Omega_{m, n+1}\right)+\int_{\beta y^{2} \leq 20} \Xi \mu \mathrm{d} y,
$$


where the quantity that remains to be controlled is now

$$
\Xi:=\frac{26+n-9 m}{10}\left\|v^{-(n+1)} \partial_{y}^{m} \partial_{\theta}^{n} v\right\|_{\mathbb{S}^{1}}^{2}-\left(1-\varepsilon_{1}\right)(1-\gamma)\left\|v^{-(n+1)} \partial_{y}^{m} \partial_{\theta}^{(n+1)} v\right\|_{\mathbb{S}^{1}}^{2} .
$$

We separate $\Xi$ by using orthogonal projection as above, using Condition [C2i] to control the term involving $\mathbb{P}\left(\partial_{y}^{m} \partial_{\theta}^{n} v\right)$, and integrating by parts in $\theta$ to control the term involving $(1-\mathbb{P})\left(\partial_{y}^{m} \partial_{\theta}^{n} v\right)$, using the fact that $\frac{26+n-9 m}{10} \leq \frac{31}{10}<4$ for all $(m, n)$ under consideration. This completes the proof.

5.2. Second-order estimates in the inner region. With Lemmas $5-7$ in hand, we are ready to prove Theorem 2 .

We begin by deriving $L_{\mu}^{2}$ bounds on all derivatives of orders two through five.

Lemma 8. Suppose that a solution $v=v(y, \theta, \tau)$ of equation (2.3) satisfies Assumption [A1] at $\tau=0$, as well as [Ca], [CO]-[C3], [COi]-[C2i], [Cg], [Cr], and [Cs] for $\tau \in\left[0, \tau_{1}\right]$. Then for the same time interval, one has

$$
\beta^{2} \Omega_{2,0}+\Omega_{1,1}+\Omega_{0,2}+\beta \Omega_{3,0}+\Omega_{2,1}+\Omega_{1,2}+\Omega_{0,3} \lesssim \beta^{4}
$$

and

$$
\beta^{\frac{4}{5}} \Omega_{4,0}+\Omega_{3,1}+\Omega_{2,2}+\Omega_{1,3}+\Omega_{0,4}+\sum_{m+n=5} \Omega_{m, n} \lesssim \beta^{4}
$$

Proof. The proof of Proposition 6.10 in [13] applies without change to establish the first estimate.

To establish the second estimate, we define

$$
\Upsilon:=\beta^{\frac{4}{5}} \Omega_{4,0}+\Omega_{3,1}+\Omega_{2,2}+\Omega_{1,3}+\Omega_{0,4}+\sum_{m+n=5} \Omega_{m, n},
$$

noting the $\beta$-weight imposed on the first term. By Lemmas 5-7 and CauchySchwarz, there exist $0<\varepsilon<C<\infty$ such that

$$
\frac{d}{d \tau} \Upsilon \leq-\varepsilon \Upsilon+C\left(\beta^{2} \Upsilon^{\frac{1}{2}}+\beta^{4}\right) .
$$

Assumption [A6] bounds $\Upsilon$ at $\tau=0$. It follows that $\Upsilon \lesssim \beta^{4}$. The result follows.

Using these estimates, we complete the proof of Theorem 2.

Proof of Theorem 2. Lemma 8 provides $L_{\mu}^{2}$ bounds on derivatives of orders two through five. To complete the proof, we use Sobolev embedding to obtain pointwise bounds on derivatives of orders two and three. Because of the weighted norm $\|\cdot\|_{\mu}$ these pointwise bounds are not uniform in $y$. Using the facts that $\mu \sim\langle y\rangle^{-\frac{6}{5}}$ as $|y| \rightarrow \infty$ and that $|y| \lesssim \beta^{-\frac{1}{2}}$ in the inner region, one obtains

$$
\beta^{2}\left(\partial_{y}^{2} v\right)^{2}+v^{-2}\left(\partial_{y} \partial_{\theta} v\right)^{2}+v^{-4}\left(\partial_{\theta}^{2} v\right)^{2} \lesssim \beta^{4-\frac{1}{10}-\frac{3}{5}}
$$

and

$$
\beta\left(\partial_{y}^{3} v\right)^{2}+v^{-2}\left(\partial_{y}^{2} \partial_{\theta} v\right)^{2}+v^{-4}\left(\partial_{y} \partial_{\theta}^{2} v\right)^{2}+v^{-6}\left(\partial_{\theta}^{3} v\right)^{2} \lesssim \beta^{4-\frac{1}{10}-\frac{3}{5}}
$$

in the inner region. These inequalities are equivalent to estimates (4.7)-(4.8). 


\section{THE SECOND BOOTSTRAP MACHINE}

In this section and those that follow, we analyze the asymptotic behavior of solutions. Specifically, we show that a solution $v$ may be decomposed into a slowlychanging "main component" and a rapidly-decaying "small component." The main component is controlled by finitely many parameters, some of which encode information about the optimal coordinate system at a given time.

We accomplish this by building a second bootstrap machine, following [13] and making modifications where necessary. Many of the arguments of [13] apply here and so do not have to be repeated in detail. Significant new arguments are needed in two areas. (I) The discrete symmetry assumptions imposed in [13] effectively fixed the cylindrical axis once and for all, which allowed us to track the behavior of the main component of the solution using only two parameters. Here, we must deal with seven parameters, corresponding to the seven-dimensional weakly unstable eigenspace of the linearization of MCF, as explained in Section 2.3. (II) We must derive improved estimates for $\left|v_{ \pm}\right|$and $\left|\left(\partial_{y} v\right)_{ \pm}\right|$in the inner region, stated as (6.10) below. These allow the first bootstrap machine to function without the discrete symmetries imposed in our earlier work.

6.1. Inputs. The second bootstrap machine requires three sets of inputs.

First we require that $u$ be a solution of equation (2.1) satisfying the following condition:

[Cd] There exists $t_{\#}>0$ such that for $0 \leq t \leq t_{\#}$, there exist $C^{1}$ functions $a(t)$, $b(t)$ and $\beta_{k}(t)(k=0, \ldots, 4)$ such that $u$ admits a decomposition

$$
\begin{aligned}
u(x, \theta, t)= & \lambda(t) v(y, \theta, \tau) \\
= & \lambda(t)\left[\left(\frac{2+b(t) y^{2}}{2-2 a(t)}\right)^{\frac{1}{2}}+\beta_{0}(t) y+\beta_{1}(t) \cos \theta+\beta_{2}(t) \sin \theta\right. \\
& \left.+\beta_{3}(t) y \cos \theta+\beta_{4}(t) y \sin \theta+\phi(y, \theta, \tau)\right]
\end{aligned}
$$

with the $L^{2}$ orthogonality properties

$$
e^{-\frac{a y^{2}}{2}} \phi \perp\left\{1, y, 1-a y^{2}, \cos \theta, \sin \theta, y \cos \theta y \sin \theta\right\},
$$

where

$$
\begin{aligned}
a(t) & :=-\lambda(t) \partial_{t} \lambda(t), \\
y & :=\lambda^{-1}(t) x, \\
\tau(t) & :=\int_{0}^{t} \lambda^{-2}(s) \mathrm{d} s, \\
\beta_{k}\left(t_{\#}\right) & =0, \quad(k=0, \ldots, 4), \\
a\left(t_{\#}\right) & =\frac{1}{2}-\frac{1}{4} b\left(t_{\#}\right) .
\end{aligned}
$$

Condition [Cd] follows from our Main Assumptions by an implicit function theorem argument highly analogous to the decomposition result proved in [14] and also used in [13]. We omit the details here. 
To state the second set of inputs, we define majorizing functions to control the decay of the quantities $\phi(y, \theta, \tau), a(t(\tau))$, and $b(t(\tau))$ that appear in equation (6.1). These are:

$$
\begin{aligned}
M_{m, n}(T) & :=\max _{\tau \leq T} \beta^{-\frac{m+n}{2}-\frac{1}{10}}(\tau)\|\phi(\cdot, \cdot, \tau)\|_{m, n}, \\
A(T) & :=\max _{\tau \leq T} \beta^{-2}(\tau)\left|a(t(\tau))-\frac{1}{2}+\frac{1}{4} b(t(\tau))\right|, \\
B(T) & :=\max _{\tau \leq T} \beta^{-\frac{3}{2}}(\tau)|b(t(\tau))-\beta(\tau)|,
\end{aligned}
$$

where $(m, n) \in\{(3,0),(11 / 10,0),(2,1),(1,1)\}$. By standard regularity theory for quasilinear parabolic equations, if the initial data satisfy the Main Assumptions [A1]-[A7] for $b_{0}$ and $c_{0}$ sufficiently small, then, making $t_{\#}>0$ smaller if necessary, the solution will satisfy the second set of inputs for this bootstrap argument, which are contained in the following condition:

[Cb] For any $\tau \leq \tau\left(t_{\#}\right)$, one has

$$
\begin{aligned}
A(\tau)+B(\tau)+|M(\tau)| & \lesssim \beta^{-\frac{1}{20}}(\tau), \\
\left|\beta_{k}(t)\right| & \lesssim \beta^{2}(\tau), k=0,1,2,3,4,
\end{aligned}
$$

where $M$ denotes the vector

$$
M:=\left(M_{i, j}\right),(i, j) \in\{(3,0),(11 / 10,0),(2,1),(1,1)\} .
$$

Remark 3. Condition [Cb] implies estimates on $v$ and $v^{-\frac{1}{2}} \partial_{y} v$ in the inner region $\beta y^{2} \leq 20$, which in turn imply Condition [C0i], the estimate $\left|\partial_{y} v\right| \lesssim \beta^{\frac{1}{2}} v^{\frac{1}{2}}$ of Condition [C1i], and the estimate $\langle y\rangle^{-1}\left|\partial_{y} v\right| \leq M^{\frac{1}{4}} \beta$ of Condition [Cg], for $M$ sufficiently large.

The final inputs to this bootstrap machine are the outputs of the first bootstrap machine, which are listed in Section 4.2. They follow from Theorems 1-4. In the sequel, we give ourselves the freedom to use all estimates detailed in Section 4.2. For the reader's convenience, we list here the implications of those estimates most commonly used in this machine: for any $\tau \in\left[0, \tau\left(t_{\#}\right)\right]$, the quantity $v$ satisfies

$$
v(y, \theta, \tau) \geq 1
$$

and there exist constants $\epsilon_{0} \ll 1$ and $C$, independent of $\tau\left(t_{\#}\right)$, such that

$$
\begin{aligned}
&\left|\partial_{y} v\right| \leq C, \\
&\left|v^{-1} \partial_{\theta}^{2} v\right|+\left|\partial_{y} \partial_{\theta}^{2} v\right| \leq \epsilon_{0} \ll 1, \\
& v^{-1}\left|\partial_{y} v\right| \leq C \beta^{\frac{1}{2}}, \quad\left|\partial_{y}^{2} v\right| \leq C \beta^{\frac{13}{20}}, \quad\left|\partial_{y}^{3} v\right| \leq C \beta^{\frac{23}{20}}, \\
& v^{-2}\left|\partial_{y} \partial_{\theta}^{2} v\right|+v^{-1}\left|\partial_{y} \partial_{\theta} v\right|+v^{-1}\left|\partial_{y}^{2} \partial_{\theta} v\right|+v^{-2}\left|\partial_{\theta}^{2} v\right|+v^{-3}\left|\partial_{\theta}^{3} v\right| \leq C \beta^{\frac{33}{20}} .
\end{aligned}
$$

6.2. Outputs. We now state the main outputs of the second bootstrap machine. They serve two main purposes. (I) The estimates stated below improve some of those in the inputs. Together with the local well-posedness of the solution, this fact enables us to continue making bootstrap arguments in larger time intervals. (II) Most importantly, the estimates below provide almost sharp control on various components of the solution, and hence a clear understanding of the geometry and asymptotic behavior of the evolving surface. 
Theorem 5. Suppose that Conditions [Cd] and [Cb] and estimates (6.5)-(6.9) hold in the interval $\tau \in\left[0, \tau\left(t_{\#}\right)\right]$. Then there exists a constant $C$ independent of $\tau\left(t_{\#}\right)$ such that in the same time interval:

(I) The parameters $a, b$, and $\beta_{k},(k=0, \ldots, 4)$ satisfy the estimates

$$
\begin{aligned}
A(\tau)+B(\tau) & \leq C, \\
\left|\beta_{0}(t)\right| & \leq C \beta^{\frac{13}{5}}, \\
\left|\beta_{1}(t)\right|+\left|\beta_{2}(t)\right| & \leq C \beta^{\frac{18}{5}}, \\
\left|\beta_{3}(t)\right|+\left|\beta_{4}(t)\right| & \leq C \beta^{\frac{13}{5}} .
\end{aligned}
$$

(II) The function $\phi$ satisfies improved estimates implied by the bound

$$
|M(\tau)| \leq C .
$$

(III) In the region $\beta y^{2} \leq 20$, one can estimate that

$$
\left|v_{ \pm}\right|+\left|\partial_{y} v_{ \pm}\right| \leq C \beta^{\frac{21}{10}}
$$

The theorem will be reformulated in Section 7 below. In that section, we provide a heuristic outline of the ideas used in its proof, with an emphasis on those that differ from what we used in [13]. The technical details of the proof appear in the appendices.

For the reader's convenience, we reformulate some implications of the estimates proved in Theorem 5 and Lemma 9 (below) in forms that are most useful for our subsequent applications.

Corollary 1. Suppose that the assumptions of Section 6.1 hold in a time interval $0 \leq \tau \leq \tau\left(t_{\#}\right)$. Then for that same time interval, the parameters $a$ and $b$ satisfy

$$
\begin{aligned}
& a(t)=\frac{1}{2}-\frac{b(t)}{4}+\mathcal{O}\left(b^{2}(t)\right), \\
& b(t)=(1+o(1)) \frac{1}{b_{0}^{-1}+\tau(t)},
\end{aligned}
$$

while the "small component" $\phi$ of the decomposition (6.1) satisfies

$$
\left\|\langle y\rangle^{-3} \phi\right\|_{L^{\infty}}+\left\|\langle y\rangle^{-2} \partial_{y} \phi\right\|_{L^{\infty}}+\left\|v^{-2} \partial_{\theta}^{2} v\right\|_{L^{\infty}} \lesssim b^{\frac{8}{5}} .
$$

Another consequence of the estimates in Theorem 5 and Lemma 9 gives control on the sequence of optimal coordinate systems we construct.

Corollary 2. Let the assumptions of Section 6.1 hold for times $0 \leq t \leq t_{\#}$, and suppose that $\left(x_{0}, x_{1}, x_{2}\right)_{n}$ and $\left(x_{0}, x_{1}, x_{2}\right)_{n+1}$ are optimal coordinates at times $t_{n}<t_{n+1}$ respectively, where $\left[t_{n}, t_{n+1}\right] \subseteq\left[0, t_{\#}\right]$. Then there exist $\Phi \in \mathbb{R}^{3}$ and $\Psi \in \mathrm{O}(3)$ such that $\left(x_{0}, x_{1}, x_{2}\right)_{n+1}=\Phi+\left(x_{0}, x_{1}, x_{2}\right)_{n} \Psi$, where

$$
|\Phi|+|\Psi-I| \lesssim b_{\text {opt }}^{2}\left(t_{n}\right) .
$$

Furthermore, for $t \in\left[t_{n}, t_{n+1}\right]$, the parameters $b$ and $\lambda$ satisfy the estimates

$$
\begin{aligned}
b(t) & =b_{\mathrm{opt}}\left(t_{n}\right)\left\{1+\mathcal{O}\left(b_{\mathrm{opt}}^{2}\left(t_{n}\right)\right)\right\}, \\
\lambda(t) & =\lambda_{\mathrm{opt}}\left(t_{n}\right)\left\{1+\mathcal{O}\left(b_{\mathrm{opt}}^{2}\left(t_{n}\right)\right)\right\} .
\end{aligned}
$$




\section{IMPROVED ESTIMATES FOR THE DECOMPOSITION}

We begin by putting the equation into a better form. Instead of studying the evolution equation for $\phi$, we fix a gauge by means of a weight factor $e^{-\frac{a}{4} y^{2}}$ chosen so that the linearization, to be obtained below, becomes a self-adjoint operator.

By Condition [Cd] in Section 6, there exists a ( $t$-scale) time $0<t_{\#} \leq \infty$ such that the gauge-fixed quantity

$$
w(y, \theta, \tau):=v(y, \theta, \tau) e^{-\frac{a}{4} y^{2}}
$$

can be decomposed as

(7.1) $w=w_{a b}(y)+e^{-\frac{a}{4} y^{2}}\left[\beta_{0} y+\beta_{1} \cos \theta+\beta_{2} \sin \theta+\beta_{3} y \cos \theta+\beta_{4} y \sin \theta\right]+\xi(y, \theta, \tau)$.

Here $w_{a b}:=V_{a, b} e^{-\frac{a}{4} y^{2}}$, and

$$
e^{-\frac{a}{4} y^{2}} \xi \perp\left\{1, y, y^{2}, \cos \theta, \sin \theta, y \cos \theta, y \sin \theta\right\},
$$

where the orthogonality is with respect to the $L^{2}\left(\mathbb{S}^{1} \times \mathbb{R}\right)$ inner product, ${ }^{11}$ and

$$
\begin{aligned}
\beta_{k}\left(t_{\#}\right) & =0, \quad(k=0,1,2,3,4), \\
a\left(t_{\#}\right) & =\frac{1}{2}-\frac{1}{4} b\left(t_{\#}\right) .
\end{aligned}
$$

The parameters $a, b$ and $\beta_{k}, k=0,1, \cdots, 4$, are $C^{1}$ functions of $t .^{12}$

We now derive the PDE satisfied by the "small component" $\xi(y, \theta, \tau)$. Below, we use this to derive the ODE satisfied by the parameters $a(\tau), b(\tau)$, and $\beta_{k}(\tau)$. Using equations (7.1) and (2.3), one finds that $\xi$ evolves by

$$
\partial_{\tau} \xi=-L(a, b) \xi+F(a, b, \beta)+N_{1}(a, b, \xi)+N_{2}(a, b, \xi)+N_{3}(a, b, \xi),
$$

where $L(a, b)$ is the linear operator

$$
L(a, b):=-\partial_{y}^{2}+\frac{a^{2}+\partial_{\tau} a}{4} y^{2}-\frac{3 a}{2}-\frac{2-2 a}{2+b y^{2}}-\frac{1}{2} \partial_{\theta}^{2} .
$$

The remaining terms on the RHS of the evolution equation (7.4) are as follows.

The quantity $F$ has two parts, $F(a, b, \beta):=F_{1}(a, b)+F_{2}(a, b, \beta)$, which are

$$
\begin{aligned}
F_{1}(a, b):=e^{-\frac{a y^{2}}{4}} & {\left[\frac{1}{\sqrt{2-2 a}} \frac{2 b}{\left(2+b y^{2}\right)^{\frac{3}{2}}}-\frac{a b y^{2}}{\sqrt{2-2 a} \sqrt{2+b y^{2}}}+a \sqrt{\frac{2+b y^{2}}{2-2 a}}\right.} \\
& \left.-\sqrt{\frac{2-2 a}{2+b y^{2}}}-\frac{\frac{1}{2} b_{\tau} y^{2}}{\sqrt{2-2 a} \sqrt{2+b y^{2}}}-\frac{\sqrt{2+b y^{2}}}{(2-2 a)^{\frac{3}{2}}} a_{\tau}\right] .
\end{aligned}
$$

and

$$
\begin{aligned}
F_{2}(a, b, \beta):= & e^{-\frac{a y^{2}}{4}}\left[\partial_{y}^{2}+V_{a, b}^{-2} \partial_{\theta}^{2}-a y \partial_{y}+a+V_{a, b}^{-2}-\partial_{\tau}\right] \\
& {\left[\beta_{0} y+\beta_{1} \cos \theta+\beta_{2} \sin \theta+\beta_{3} y \cos \theta+\beta_{4} y \sin \theta\right] . }
\end{aligned}
$$

\footnotetext{
${ }^{11}$ In the remainder of this paper, the inner product we use is $\langle\cdot, \cdot\rangle$. As defined in Section 2.1, this is the standard (unweighted) inner product for $L^{2} \equiv L^{2}\left(\mathbb{S}^{1} \times \mathbb{R}\right)$.

${ }^{12}$ To simplify notation, we mildly abuse notation by writing $a(\tau), b(\tau)$, and $\beta_{k}(\tau)$ for $a(t(\tau))$, $b(t(\tau))$, and $\beta_{k}(t(\tau))$, respectively. This will not cause confusion, as the original functions $a(t)$, $b(t)$, and $\beta_{k}(t)$ are not needed in what follows.
} 
The first nonlinear term $N_{1}$ is

$$
N_{1}(a, b, \xi):=-\frac{1}{v} \frac{2-2 a}{2+b y^{2}} e^{\frac{a y^{2}}{4}} \tilde{\xi}^{2},
$$

where

$$
\tilde{\xi}:=e^{-\frac{a y^{2}}{4}}\left[e^{\frac{a y^{2}}{4}} \xi+\beta_{0} y+\beta_{1} \cos \theta+\beta_{2} \sin \theta+\beta_{3} \cos \theta y+\beta_{4} \sin \theta y\right] .
$$

The second nonlinear term $N_{2}$ is

$$
N_{2}(a, b, \xi):=-e^{-\frac{a y^{2}}{4}} \frac{p^{2}}{1+p^{2}+q^{2}} \partial_{y}^{2} v .
$$

The final nonlinear term $N_{3}$ is

$$
N_{3}(a, b, \xi):=N_{3,1}(a, b, \xi)+N_{3,2}(a, b, \xi),
$$

where

$$
N_{3,1}:=\left[V_{a, b}^{-2}-\frac{1}{2}\right] \partial_{\theta}^{2} \xi
$$

and

$$
\begin{aligned}
N_{3,2}:=\left[v^{-2} \frac{1+p^{2}}{1+q^{2}+p^{2}}-V_{a, b}^{-2}\right] & \partial_{\theta}^{2} v e^{-\frac{a y^{2}}{4}} \\
& -e^{-\frac{a y^{2}}{4}} v^{-1} \frac{2 p q}{1+p^{2}+q^{2}} \partial_{\theta} \partial_{y} v+e^{-\frac{a y^{2}}{4}} v^{-2} \frac{q}{1+p^{2}+q^{2}} \partial_{\theta} v .
\end{aligned}
$$

7.1. The finite-dimensional part of the decomposition. We next derive and estimate ODE for the parameters $a, b$ and $\beta_{k},(k=0, \cdots, 4)$ by using the evolution equation (7.4) for $\xi$ and the orthogonality conditions (7.2). Before providing the details of the technical proof, we illustrate the key ideas involved by heuristically outlining how one derives and estimates an ODE for $\beta_{0}$.

Taking the $L^{2}\left(\mathbb{S}^{1} \times \mathbb{R}\right)$ inner product of $(7.4)$ with the function $y e^{-\frac{a}{4} y^{2}}$ yields

$$
\left\langle y e^{-\frac{a}{4} y^{2}}, \partial_{\tau} \xi\right\rangle=-\left\langle y e^{-\frac{a}{4} y^{2}}, L(a, b) \xi\right\rangle+\left\langle y e^{-\frac{a}{4} y^{2}}, F(a, b, \beta)\right\rangle+\cdots .
$$

The fact that $\xi \perp y e^{-\frac{a}{4} y^{2}}$ in (7.2) implies that

$$
\left|\left\langle y e^{-\frac{a}{4} y^{2}}, \partial_{\tau} \xi\right\rangle\right|=\left|\partial_{\tau}\left\langle y e^{-\frac{a}{4} y^{2}}, \xi\right\rangle+\frac{a_{\tau}}{4}\left\langle y^{3} e^{-\frac{a}{4} y^{2}}, \xi\right\rangle\right| \lesssim\left|a_{\tau}\right|\left\|\langle y\rangle^{-3} e^{\frac{a}{4} y^{2}} \xi\right\|_{L^{\infty}} .
$$

Noting that $y e^{-\frac{a}{4} y^{2}}$ is an eigenfunction of the self-adjoint operator

$$
-\partial_{y}^{2}-\frac{1}{2} \partial_{\theta}^{2}+\frac{a^{2}}{4} y^{2}-2 a-\frac{1}{2}
$$

that constitutes the "main part" of the linear operator $L(a, b)$ appearing in (7.4), we obtain

$$
\left|\left\langle y e^{-\frac{a}{4} y^{2}}, L(a, b) \xi\right\rangle\right| \lesssim\left(\left|a_{\tau}\right|+b\right)\left\|\langle y\rangle^{-3} e^{\frac{a}{4} y^{2}} \xi\right\|_{L^{\infty}} .
$$

Because $y e^{-\frac{a}{4} y^{2}}$ is an odd function of $y$ and is independent of $\theta$, we then get

$$
\left\langle y e^{-\frac{a}{4} y^{2}}, F(a, b, \beta)\right\rangle=2 \pi\left[\Omega_{1}(a, b) \beta_{0}-\partial_{\tau} \beta_{0}\right] \int_{-\infty}^{\infty} y^{2} e^{-\frac{a}{2} y^{2}} \mathrm{~d} y,
$$

where $\Omega_{1}$ is the positive scalar function

$$
\Omega_{1}(a, b):=\frac{\int_{-\infty}^{\infty} y^{2} V_{a, b}^{-2} e^{-\frac{a}{2} y^{2}} \mathrm{~d} y}{\int_{-\infty}^{\infty} y^{2} e^{-\frac{a}{2} y^{2}} \mathrm{~d} y} \approx \frac{1}{2} .
$$


Here we used the fact that $V_{a, b}=\sqrt{\frac{2+b y^{2}}{2-2 a}} \approx \frac{1}{2}$, which is implied by $a \approx \frac{1}{2}$ and the fact that $b$ is positive and small. Collecting the estimates above yields the desired equation for $\beta_{0}$, which has the form

$$
\partial_{\tau} \beta_{0}-\Omega_{1}(a, b) \beta_{0}=\mathcal{O}\left(\left(\left|a_{\tau}\right|+|b|\right)\left\|\langle y\rangle^{-3} e^{\frac{a}{4} y^{2}} \xi\right\|_{L^{\infty}}\right)+\cdots .
$$

Finally, we sketch how one derives an estimate for $\beta_{0}$ based on this ODE. Using the boundary condition $\beta_{0}\left(\tau\left(t_{\#}\right)\right)=0$ from (7.3), we can rewrite the equation for $\beta_{0}$ at any $\tau \leq \tau\left(t_{\#}\right)$ as

$\beta(\tau)=\int_{\tau}^{\tau\left(t_{\#}\right)}\left\{e^{-\int_{s}^{\tau\left(t_{\#}\right)} \Omega_{1}(a, b)\left(s_{1}\right) d s_{1}} \mathcal{O}\left(\left(\left|a_{\tau}\right|+|b|\right)\left\|\langle y\rangle^{-3} e^{\frac{a}{4} y^{2}} \xi\right\|_{L^{\infty}}\right)(s)+\cdots\right\} \mathrm{d} s$.

Using the fact that $\Omega_{1} \approx \frac{1}{2}$ and the smallness of $\left|a_{\tau}\right|, b$, and $\left\|\langle y\rangle^{-3} e^{\frac{a}{4} y^{2}} \xi\right\|_{L^{\infty}}$, this implies the desired estimate.

Using the method outlined above, we obtain the following estimates.

Lemma 9. For all times that the assumptions of Section 6.1 hold, one has

$$
\begin{aligned}
-\frac{2}{1-a} \partial_{\tau} a+2 b+4(2 a-1) & =\mathcal{O}\left(\beta^{\frac{5}{2}}\right), \\
\partial_{\tau} b+b^{2} & =\mathcal{O}\left(\beta^{\frac{5}{2}}\right), \\
\partial_{\tau} \beta_{0} & =\Omega_{1}(a, b) \beta_{0}+\mathcal{O}\left(\beta^{\frac{5}{2}}\right), \\
\partial_{\tau} \beta_{1} & =a \beta_{1}+(\text { Small })_{1}, \\
\partial_{\tau} \beta_{2} & =a \beta_{2}+(\text { Small })_{2}, \\
\partial_{\tau} \beta_{3} & =(\text { Small })_{3}, \\
\partial_{\tau} \beta_{4} & =(\text { Small })_{4},
\end{aligned}
$$

where the terms $(\text { Small })_{\ell},(\ell=1, \ldots, 4)$, satisfy

$$
\mid(\text { Small })_{\ell} \mid \lesssim \min \left\{\beta^{\frac{16}{5}}, \beta^{\frac{18}{5}}+\beta^{\frac{31}{20}}\left\|\langle y\rangle^{-5} \partial_{\theta} \xi e^{\frac{a y^{2}}{4}}\right\|_{L^{\infty}}\right\} .
$$

Furthermore, one has

$$
\left|2 a-1+\frac{1}{2} b\right| \lesssim \beta^{2} \quad \text { and } \quad|b-\beta| \lesssim \beta^{\frac{3}{2}},
$$

(i.e. $A+B \lesssim 1)$ and

$$
\begin{aligned}
&\left|\beta_{0}\right| \lesssim \beta^{\frac{5}{2}} \\
&\left|\beta_{1}\right|+\left|\beta_{2}\right| \lesssim \min \left\{\beta^{\frac{16}{5}},\left(1+M_{4}\right) \beta^{\frac{18}{5}}\right\}, \\
&\left|\beta_{3}\right|+\left|\beta_{4}\right| \lesssim \min \left\{\beta^{\frac{11}{5}},\left(1+M_{4}\right) \beta^{\frac{13}{5}}\right\} .
\end{aligned}
$$

The function $M_{4}$ above is defined as

$$
M_{4}(\tau):=\max _{0 \leq s \leq \tau} \beta^{-\frac{21}{10}}\left\|\langle y\rangle^{-5} \partial_{\theta} \xi(\tau) e^{\frac{a y^{2}}{4}}\right\|_{L^{\infty}} .
$$

We prove the lemma in Appendix A. 
7.2. The infinite-dimensional part of the decomposition. We next show how to control the infinite-dimensional part $\xi$. Many of the arguments here are virtually identical to their counterparts in [13]. Before providing details, we again begin with a heuristic outline of the main ideas behind the proof.

We apply Duhamel's principle to equation (7.4) for $\xi$ to obtain

$$
\xi(\tau)=U(\tau, 0) \xi(0)+\int_{0}^{\tau} U(\tau, \sigma) \cdots \mathrm{d} \sigma
$$

where $U(\tau, \sigma)$ is the propagator generated by the linear operator $-L(a, b)$ from time $\sigma$ to $\tau$. To prove the desired decay estimates for $\xi$, we exploit the decay of $U(\tau, \sigma)$ in a suitable subspace of $L^{\infty}$. To achieve this, we must overcome two difficulties. (I) The generator of $U(\tau, \sigma)$ namely $L(a, b)$, has a seven-dimensional nonpositive eigenspace, spanned by $e^{-\frac{a}{4} y^{2}}, y e^{-\frac{a}{4} y^{2}},\left(a y^{2}-1\right) e^{-\frac{a}{4} y^{2}},(\cos \theta) e^{-\frac{a}{4} y^{2}},(\sin \theta) e^{-\frac{a}{4} y^{2}}$, $(y \cos \theta) e^{-\frac{a}{4} y^{2}}$, and $(y \sin \theta) e^{-\frac{a}{4} y^{2}}$. This implies that $U(\tau, \sigma)$ may grow in these directions. (II) The operator $L(a, b)$ is not autonomous, so $U(\tau, \sigma) \neq e^{-t L(a, b)}$.

We overcome the first difficulty by using the fact that $\xi$ is orthogonal to the unstable eigenspace. At least intuitively, one expects $U(\tau, \sigma)$ to decay exponentially fast in directions orthogonal to that space. To overcome the second difficulty, we make another gauge change, reparameterizing the function $\xi$ to obtain a new function whose evolution is dominated by an autonomous operator.

Using these ideas, one first proves the following.

Lemma 10. For all times that the assumptions of Section 6.1 hold, one has

$$
\left|M_{m, n}\right| \lesssim 1, \quad \text { for } \quad(m, n) \in\{(3,0),(11 / 10,0),(2,1),(1,1)\} .
$$

The proof is essentially identical to that of Proposition 9.2 from [13], because it relies only on the outputs of the first bootstrap machine, hence does not need the discrete symmetry assumptions that were in force there. So we omit it here.

Next we turn to estimating the functions $v_{ \pm}$and $\left(\partial_{y} v\right)_{ \pm}$defined by formula $(2.9)$. These estimates represent a departure from [13]. In what follows, we present the difficulties and the ideas used to overcome them when estimating $v_{ \pm}$. The arguments for $\left(\partial_{y} v\right)_{ \pm}$are very similar, hence omitted. Our objective is to prove the following.

Lemma 11. For all times that the assumptions of Section 6.1 hold, $M_{4} \lesssim 1$, i.e.

$$
\left\|\langle y\rangle^{-5} \partial_{\theta} \xi e^{\frac{a y^{2}}{4}}\right\|_{L^{\infty}} \lesssim \beta^{\frac{21}{10}} .
$$

Lemma 12. For all times that the assumptions of Section 6.1 hold, in the region $\beta y^{2} \leq 20$, one has

$$
\left|e^{\frac{a y^{2}}{4}} \xi_{ \pm}\right|+\left|\left(\partial_{y} e^{\frac{a y^{2}}{4}} \xi\right)_{ \pm}\right| \lesssim \beta^{\frac{21}{10}}
$$

Lemmas 11 and 12 are proved in Appendices B and C, respectively.

The difficulties encountered in their proofs are as follows. The decomposition of $v$ implies that

$$
\left|v_{ \pm}\right| \leq\left|\beta_{1}\right|+\left|\beta_{2}\right|+\left|\beta_{3} y\right|+\left|\beta_{4} y\right|+\left|\xi_{ \pm}\right| e^{\frac{a y^{2}}{4}}
$$

where the $\theta$-independent functions $\xi_{ \pm}$are defined using (2.9). For the purposes of Lemmas 11 and 12 , the estimates $\left|\beta_{3}\right|+\left|\beta_{4}\right|=\mathcal{O}\left(\beta^{\frac{11}{5}}\right)$ from (7.14) are obviously not good enough. In the region $b y^{2} \leq 20$, they only give $\left|\beta_{k} y\right| \lesssim \beta^{\frac{8}{5}}$, which is 
significantly slower than the desired $\beta^{\frac{21}{10}}$ decay. The estimates for $\xi$ in Lemma 10 are not good enough either. As noted above, the reason we want to prove stronger decay is so that the first bootstrap machine can function without needing discrete symmetry hypotheses. So we must derive improved estimates for $\left|\partial_{\theta} \xi\right|$. But it is not difficult to see that $\partial_{\theta} \xi$ admits better decay estimates than does $\xi$. Differentiation allows us to remove the slowly-decaying $\theta$-independent components of the solution, and thereby to obtain improved estimates.

In the remainder of this section, we outline the main ideas used in proving estimate (7.16) in Appendix C. The steps used to prove (7.15) in Appendix B are similar, hence will not be discussed here.

Using (7.4) and recalling definition (2.9), we compute that

$$
\partial_{\tau} \xi_{ \pm}=-L_{0}(a) \xi_{ \pm}+\cdots
$$

where

$$
L_{0}(a):=-\partial_{y}^{2}+\frac{a^{2}+\partial_{\tau} a}{4} y^{2}-\frac{3 a}{2} .
$$

Our strategy for estimating $\xi_{ \pm}$is similar to that used to estimate $\xi$ in the proof of Lemma 10, and hence has similar difficulties: the linear operator $L_{0}(a)$ is timedependent and has nonpositive eigenvalues with eigenvectors $e^{-\frac{a y^{2}}{4}}$ and $y e^{-\frac{a y^{2}}{4}}$. To take care of these eigendirections, we use the orthogonality conditions imposed on $\xi$ in (7.2) to see that $\xi_{ \pm} \perp \operatorname{span}\left\{e^{-a y^{2} / 4}, y e^{-a y^{2} / 4}\right\}$. The intuition behind the argument is that we obtain good estimates for $\xi_{ \pm}$by applying $L_{0}(a)$ to the orthogonal complement of the finite-dimensional unstable subspace. We make this rigorous in Appendix C. Once that work is done, our final result follows readily:

Proof of estimate (6.10) in Theorem 5. We use the decomposition of $v$ in (6.1) to relate $\xi_{ \pm}$to $v_{ \pm}$, obtaining

$$
\left|v_{ \pm}\right| \leq\left|\beta_{1}\right|+\left|\beta_{2}\right|+\left|\beta_{3} y\right|+\left|\beta_{4} y\right|+\left|\xi_{ \pm}\right| e^{\frac{a y^{2}}{4}} .
$$

The estimate for $\xi_{ \pm}$in Lemma 12 and the estimates for $\beta_{k}(k=1, \ldots, 4)$ in Lemma 9 then imply that $\left|v_{ \pm}(y, \theta, \tau)\right| \lesssim \beta^{\frac{21}{10}}(\tau)$.

The estimate for $\left(\partial_{y} v\right)_{ \pm}$is obtained similarly.

\section{Proof of the Main Theorem}

In this section, we collect the remaining arguments needed to complete the proof of our Main Theorem, modulo the technical details collected in the appendices.

Proof of the Main Theorem. By placing an Angenent self-similarly shrinking torus around the (approximate) center of the neck, one sees easily that the solution must become singular before some time $T^{*}<\infty$. So suppose that $\left[0, T_{*}\right)$ is the maximal time interval such that for any time $t \in\left[0, T_{*}\right) \subseteq\left[0, T^{*}\right)$, we can construct an optimal coordinate system in which

$$
u(x, \theta, t)=\lambda_{\mathrm{opt}}(t)\left\{\sqrt{\frac{2+b_{\mathrm{opt}}(t) y^{2}}{1+\frac{1}{2} b_{\mathrm{opt}}(t)}}+\phi_{\mathrm{opt}}(y, \theta, t)\right\},
$$

where $y=\lambda_{\text {opt }}^{-1} x$, and $\phi$ satisfies the orthogonality conditions of Definition 1, along with the estimate

$$
\left\|\langle y\rangle^{-3} \phi_{\mathrm{opt}}(\cdot, \cdot, t)\right\|_{L^{\infty}} \lesssim b_{\mathrm{opt}}^{8 / 5}(t) .
$$


In this case, we claim that for any sequence of times $t_{n} \nearrow T_{*}$ at which we construct optimal coordinate systems, one has $b_{\text {opt }}\left(t_{n}\right) \rightarrow 0$ and $\lambda_{\text {opt }}\left(t_{n}\right) \rightarrow 0$ as $n \rightarrow \infty$. By the estimates in Theorem 5 (see also Corollaries 1-2) for the components of the decomposition (8.1), this implies that the surface must become singular as $t \nearrow T_{*}$.

We prove the claim by contradiction, showing that if either quantity $b_{\text {opt }}\left(t_{n}\right)$ or $\lambda_{\text {opt }}\left(t_{n}\right)$ has a positive lower bound, then the other does also, which implies that the solution can be extended past $T_{*}$, contradicting the assumption that $T_{*}$ is maximal. We provide a detailed argument for the case that there exists a constant $c_{\infty}>0$ such that $b_{\text {opt }}\left(t_{n}\right) \geq c_{\infty}$. (An analogous argument works if $\lambda_{\text {opt }}\left(t_{n}\right) \geq c_{\infty}^{\prime}$.)

With respect to an optimal coordinate system constructed at $t_{n}$, there exists a time interval $\left[t_{n-1}, t_{n}\right] \subseteq\left[0, t_{n}\right]$ in which the solution $v=\lambda^{-1} u$ can be parameterized as in (2.10). Using the fact that $\partial_{\tau} \log \lambda=-a$, and the boundary conditions stipulated in Definition 1 that ensure that $\lambda_{\text {opt }}=\lambda$ at any times at which we construct optimal coordinates, we see that

$$
\lambda_{\text {opt }}\left(t_{n}\right)=e^{-\int_{t_{n-1}}^{t_{n}} a(\hat{\tau}) \mathrm{d} \hat{\tau}} \lambda_{\text {opt }}\left(t_{n-1}\right) .
$$

Then using the upper bound for $b$ in Corollary 1 , the consequence of estimate (7.12) that $a=\frac{1}{2}+\mathcal{O}(b)$ is bounded independently of $n$, and the upper bound $T_{*} \leq T^{*}$, we conclude that there exists $c>0$ independent of $n$ such that $\lambda_{\text {opt }}\left(t_{n}\right) \geq c \lambda_{\text {opt }}(0)$. Because $n$ was arbitrary, this contradicts the maximality of $T_{*}$ and proves the claim.

Part (I) of the theorem follows directly from the claim.

Part (II) then follows from Corollary 2, because the claim implies that we can construct optimal coordinate systems up to the singular time.

Proving Part (III) takes more work. Obtaining asymptotics for the sequential parameters $\lambda_{\text {opt }}\left(t_{n}\right)$ and $b_{\text {opt }}\left(t_{n}\right)$ that determine the "main components" of the solution's decomposition is complicated by the fact that we do not have ODE for them; we only have ODE for the quantities $\lambda$ and $b$ that depend smoothly on the sequential choices of $\lambda_{\text {opt }}\left(t_{n}\right)$ and $b_{\text {opt }}\left(t_{n}\right)$. So we proceed as follows. Working in a time interval $\left[t_{n}, t_{m}\right]$, we use the relation $\lambda \partial_{t} \lambda=-a$ to see that

$$
\lambda^{2}(t)=\lambda_{\text {opt }}^{2}\left(t_{m}\right)+\int_{t}^{t_{m}} 2 a(s) \mathrm{d} s
$$

By the estimates for $a$ in Corollary 1 and for $b$ in Corollary 2, this implies that

$$
\lambda_{\text {opt }}^{2}\left(t_{n}\right)=\left(1+\mathcal{O}\left(b_{\text {opt }}\left(t_{m}\right)\right)\left\{\lambda_{\text {opt }}^{2}\left(t_{m}\right)+\left(t_{m}-t_{n}\right)\right\} .\right.
$$

Letting $m \rightarrow \infty$ and using the fact proved above that $\lambda_{\text {opt }}\left(t_{m}\right) \searrow 0$ as $m \rightarrow \infty$, we conclude that the asymptotic behavior of $\lambda_{\text {opt }}\left(t_{n}\right)$ as $n \rightarrow \infty$ is

$$
\lambda_{\text {opt }}\left(t_{n}\right)=(1+o(1)) \sqrt{T-t_{n}} .
$$

Next, by the estimate for $a$ in Corollary 1 and the fact that $\frac{d \tau}{d \lambda}=-\frac{1}{a}$, we find that as $n \rightarrow \infty$, one has

$$
\tau\left(t_{n}\right)=(1+o(1)) \log \frac{1}{T-t_{n}} .
$$

Then using the estimates for $b$ and $b_{\text {opt }}$ in Corollaries $1-2$, it follows that as $n \rightarrow \infty$,

$$
b_{\mathrm{opt}}\left(t_{n}\right)=(1+o(1))\left(\log \frac{1}{T-t_{n}}\right)^{-1}
$$

Equations (8.2) and (8.3) establish Part (III) of the theorem. 
Part (IV) of the theorem follows directly from the outputs of the first and second bootstrap machines, as stated in Sections 4.2 and 6.2, respectively.

The proof is complete.

\section{Appendix A. Proof of Lemma 9}

In this appendix, we prove Lemma 9. To avoid unenlightening repetition, we provide detailed arguments only for the $a, b, \beta_{0}$, and $\beta_{3}$ evolution equations and their estimates. The arguments for the others are almost identical.

A.1. Proofs of estimates (7.8) and (7.9). The derivations of (7.8) and (7.9) are almost identical to those in our previous work [13], hence are only sketched here.

We rewrite $F_{1}(a, b)$ in the form

$$
\begin{aligned}
F_{1}(a, b) & =e^{-\frac{a y^{2}}{4}} \frac{1}{\sqrt{2-2 a} \sqrt{2+b y^{2}}}\left[\frac{2 b}{2+b y^{2}}+4 a-2-\frac{1}{2} b_{\tau} y^{2}-\frac{1}{1-a} a_{\tau}-\frac{a_{\tau} b y^{2}}{2-2 a}\right] \\
& =e^{-\frac{a y^{2}}{4}} \frac{1}{\sqrt{2-2 a} \sqrt{2+b y^{2}}}\left[\Gamma_{1}-\frac{1}{2} \Gamma_{2} y^{2}+\Gamma_{3}\right],
\end{aligned}
$$

with $\Gamma_{k}(k=1,2,3)$ defined as

$$
\begin{aligned}
& \Gamma_{1}:=b+4 a-2-\frac{1}{1-a} a_{\tau}, \\
& \Gamma_{2}:=b^{2}+\partial_{\tau} b, \\
& \Gamma_{3}:=\frac{b^{3} y^{4}}{2\left(2+b y^{2}\right)}-\frac{a_{\tau} b y^{2}}{2-2 a} .
\end{aligned}
$$

Then we take an inner product of (7.4) with the functions $e^{-\frac{a}{4} y^{2}}$ and $\left(a y^{2}-1\right) e^{-\frac{a}{4} y^{2}}$, applying the orthogonality conditions of (7.2) to obtain

$$
\left|\Gamma_{1}\right|+\left|\Gamma_{2}\right| \lesssim \beta^{\frac{5}{2}}
$$

Recalling the definitions of $\Gamma_{1}$ and $\Gamma_{2}$ above, it is easy to see that this estimate implies (7.8) and (7.9).

\section{A.2. Proof of the estimates in (7.12).}

Proof of the estimates in (7.12). We write $\Gamma_{1}$ as

$$
\Gamma_{1}=\frac{1}{1-a}\left[2 \tilde{\Gamma}_{1}-\partial_{\tau} \tilde{\Gamma}_{1}+\frac{1}{4} \partial_{\tau} b-2 \tilde{\Gamma}_{1}^{2}+\frac{1}{8} b^{2}\right]
$$

with $\tilde{\Gamma}_{1}:=a-\frac{1}{2}+\frac{1}{4} b$. To estimate the various components, we use the assumptions

$$
\max _{s \leq \tau}|b(s)-\beta(s)| \beta^{-\frac{3}{2}}(s)=B(\tau) \lesssim \beta^{-\frac{1}{20}}(\tau)
$$

and

$$
\max _{s \leq \tau}\left|a-\frac{1}{2}+\frac{1}{4} b\right| \beta^{-\frac{5}{2}}(s)
$$

to obtain $b \leq 2 \beta$ and $\left|\tilde{\Gamma}_{1}\right| \lesssim \beta^{2}$. Hence we get

$$
\left|\partial_{\tau} \tilde{\Gamma}_{1}-2 \tilde{\Gamma}_{1}\right| \lesssim \beta^{2}
$$

By the boundary condition $a\left(\tau\left(t_{\#}\right)\right)=\frac{1}{2}-\frac{1}{4} b\left(\tau\left(t_{\#}\right)\right)$ in (7.3), we have

$$
\tilde{\Gamma}_{1}\left(\tau_{\#}\right)=0
$$


where we denote $\tau\left(t_{\#}\right)$ by $\tau_{\#}$. We rewrite (A.2) as

$$
\begin{aligned}
\left|\tilde{\Gamma}_{1}(\tau)\right| & \lesssim \int_{\tau}^{\tau_{\#}} e^{2(\tau-s)} \beta^{2}(s) \mathrm{d} s \\
& \lesssim \beta^{2}(\tau) \int_{\tau}^{\tau_{\#}} e^{2(\tau-s)} \mathrm{d} s \\
& \lesssim \beta^{2}(\tau),
\end{aligned}
$$

and then we use the definition of $A$ in (6.3) to obtain the first estimate in (7.12).

The argument used to estimate $b$ is identical to the corresponding argument from [13]. We rewrite $\Gamma_{2}$ and use estimate (A.1) to see that

$$
\left|\partial_{\tau} \frac{1}{b}-1\right|=\left|\partial_{\tau}\left[\frac{1}{b}-\frac{1}{\beta}\right]\right| \lesssim \beta^{\frac{1}{2}} .
$$

Recall that $\beta:=\left(b_{0}^{-1}+\tau\right)^{-1}$. It is clear that $\left[\frac{1}{b}-\frac{1}{\beta}\right]_{\tau=0}=0$, and consequently that

$$
\left|\frac{1}{b}-\frac{1}{\beta}\right|(\tau) \lesssim \int_{0}^{\tau} \beta^{\frac{1}{2}}(\tau) d \tau \lesssim \beta^{-\frac{1}{2}}(\tau) .
$$

Finally, recalling the definition of $B$ in (6.4), we obtain $|b(\tau)-\beta(\tau)| \lesssim \beta^{\frac{3}{2}}(\tau)$ and $B \lesssim 1$

The following facts will be used frequently in the rest of the paper.

Lemma 13. For all times that the assumptions in Section 6.1 hold, one has

$$
\left|a_{\tau}\right|+\left|b_{\tau}\right| \lesssim \beta^{2}
$$

and

$$
1-\beta^{\frac{1}{4}} \leq \frac{b}{\beta} \leq 1+\beta^{\frac{1}{4}} .
$$

Proof. To obtain estimate (A.4), we combine the estimate for $b_{\tau}$ from (A.1), the estimate for $a_{\tau}$ from (A.2), and the estimate $\tilde{\Gamma}_{1}=\mathcal{O}\left(\beta^{2}\right)$ from (A.3).

Estimate (A.5) follows from the observation that

$$
\max _{s \leq \tau}|b(s)-\beta(s)| \beta^{-\frac{3}{2}}(s)=B(\tau) \lesssim 1 .
$$

A.3. Proofs of estimates (7.10) and (7.13). We start with proving (7.10), using methods outlined in the discussion before Lemma 9.

Proof of estimate (7.10). Following the approach outlined in our introduction to Lemma 9, we take the inner product of (7.4) with $y e^{-\frac{a y^{2}}{4}}$ to obtain

$$
\left\langle y e^{-\frac{a y^{2}}{4}}, \partial_{\tau} \xi\right\rangle=\sum_{k=1}^{5} A_{k},
$$

where the terms on the RHS are defined by

$$
\begin{aligned}
A_{1} & :=-\left\langle y e^{-\frac{a y^{2}}{4}},\left[-\partial_{y}^{2}+\frac{a^{2}+a_{\tau}}{4} y^{2}-\frac{3 a}{2}-\frac{1}{2} \partial_{\theta}^{2}-\frac{\frac{1}{2}+a}{2+b y^{2}}\right] \xi\right\rangle+\left\langle y e^{-\frac{a y^{2}}{4}}, N_{3,1}\right\rangle \\
& =-\left\langle y e^{-\frac{a y^{2}}{4}},\left[-\partial_{y}^{2}+\frac{a^{2}+a_{\tau}}{4} y^{2}-\frac{3 a}{2}-V_{a, b}^{-2} \partial_{\theta}^{2}-\frac{\frac{1}{2}+a}{2+b y^{2}}\right] \xi\right\rangle,
\end{aligned}
$$


and

$$
\begin{aligned}
& A_{2}:=\left\langle y e^{-\frac{a y^{2}}{4}}, F(a, b, \beta)\right\rangle, \\
& A_{3}:=\left\langle y e^{-\frac{a y^{2}}{4}}, N_{1}\right\rangle, \\
& A_{4}:=\left\langle y e^{-\frac{a y^{2}}{4}}, N_{2}\right\rangle, \\
& A_{5}:=\left\langle y e^{-\frac{a y^{2}}{4}}, N_{3,2}\right\rangle .
\end{aligned}
$$

We now show how the terms in equation (A.6) are controlled.

For the LHS of (A.6), we use the orthogonality condition $\xi \perp y e^{-\frac{a y^{2}}{4}}$ in (7.2) to obtain

$$
\begin{aligned}
\left\langle y e^{-\frac{a y^{2}}{4}}, \partial_{\tau} \xi\right\rangle & =\partial_{\tau}\left\langle y e^{-\frac{a y^{2}}{4}}, \xi\right\rangle+\frac{a_{\tau}}{4}\left\langle y^{3} e^{-\frac{a y^{2}}{4}}, \xi\right\rangle \\
& =\mathcal{O}\left(\left|a_{\tau}\right|\left\|\langle y\rangle^{-3} e^{\frac{a y^{2}}{4}} \xi\right\|_{L^{\infty}}\right) \\
& =\mathcal{O}\left(\beta^{\frac{71}{20}}\right) .
\end{aligned}
$$

Then we use the estimate $\left|a_{\tau}\right|=\mathcal{O}\left(\beta^{2}\right)$ from (A.4), and the assumption that $M_{3,0} \lesssim$ $\beta^{-\frac{1}{20}}$ to obtain

$$
\left\|\langle y\rangle^{-3} e^{\frac{a y^{2}}{4}} \xi\right\|_{L^{\infty}} \lesssim \beta^{\frac{8}{5}} M_{3,0} \lesssim \beta^{\frac{31}{20}} .
$$

In $A_{1}$, many terms cancel because of the fact that $\xi \perp y e^{-\frac{a y^{2}}{4}}$, where $y e^{-\frac{a y^{2}}{4}}$ is an eigenfunction of the self-adjoint operator $-\partial_{y}^{2}+\frac{a^{2}}{4} y^{2}-V_{a, b}^{-2} \partial_{\theta}^{2}$. We compute that

$$
\begin{aligned}
A_{1}= & -\left\langle y e^{-\frac{a y^{2}}{4}},\left[-\partial_{y}^{2}+\frac{a^{2}}{4} y^{2}-\frac{3 a}{2}-V_{a, b}^{-2} \partial_{\theta}^{2}-\frac{\frac{1}{2}+a}{2}\right] \xi\right\rangle \\
& -\left\langle y e^{-\frac{a y^{2}}{4}}, \frac{a_{\tau}}{4} y^{2} \xi\right\rangle+\left\langle y e^{-\frac{a y^{2}}{4}},\left[\frac{\frac{1}{2}+a}{2+b y^{2}}-\frac{\frac{1}{2}+a}{2}\right] \xi\right\rangle \\
= & -\left\langle y e^{-\frac{a y^{2}}{4}}, \frac{a_{\tau}}{4} y^{2} \xi\right\rangle-\left(\frac{1}{2}+a\right) b\left\langle y e^{-\frac{a y^{2}}{4}}, \frac{y^{2}}{2\left(2+b y^{2}\right)} \xi\right\rangle .
\end{aligned}
$$

Hence we conclude that

$$
\left|A_{1}\right| \lesssim\left(\left|a_{\tau}\right|+b\right)\left\|\langle y\rangle^{-3} e^{\frac{a y^{2}}{4}} \xi\right\|_{L^{\infty}} \lesssim \beta^{\frac{51}{20}} .
$$

The expression for $A_{2}$ can be simplified by observing that $y e^{-\frac{a y^{2}}{4}}$ is odd in $y$ and independent of $\theta$, hence is orthogonal to the functions even in $y$ or $\theta$-dependent. Thus we compute that

$$
\begin{aligned}
A_{2} & =\left\langle y e^{-\frac{a y^{2}}{4}}, F_{1}(a, b)+F_{2}(a, b, \beta)\right\rangle \\
& =\left\langle y e^{-\frac{a y^{2}}{4}}, e^{-\frac{a y^{2}}{4}}\left[\partial_{y}^{2}-a y \partial_{y}+a+V_{a, b}^{-2}-\partial_{\tau}\right] \beta_{0} y\right\rangle .
\end{aligned}
$$

Consequently, we get

$$
A_{2}=2 \pi\left[\Omega_{1}(a, b) \beta_{0}-\partial_{\tau} \beta_{0}\right] \int_{-\infty}^{\infty} y^{2} e^{-\frac{a y^{2}}{2}} \mathrm{~d} y,
$$

where $\Omega_{1}(a, b)$ is the constant defined in (7.7). 
For $A_{3}$, we use the definition of $\tilde{\xi}$ in (7.5) and the assumptions in Condition [Cb] that $\left|\beta_{k}\right| \lesssim \beta^{2}$ for $k=0,1, \ldots, 4$ to obtain

$$
\begin{aligned}
A_{3} & \lesssim\left\|\langle y\rangle^{-6} e^{\frac{a y^{2}}{2}} \tilde{\xi}^{2}\right\|_{L^{\infty}} \\
& \lesssim\left\|\langle y\rangle^{-3} e^{\frac{a y^{2}}{2}} \xi^{2}\right\|_{L^{\infty}}^{2}+\sum_{k=0}^{4}\left|\beta_{k}\right|^{2} \\
& \lesssim \beta^{3} .
\end{aligned}
$$

For $A_{4}$, direct calculation yields

$$
\left|A_{4}\right| \lesssim\left\|\langle y\rangle^{-1} p\right\|_{L^{\infty}}^{2}\left\|\partial_{y}^{2} v\right\|_{L^{\infty}}=\left\|\langle y\rangle^{-1} \partial_{y} v\right\|_{L^{\infty}}^{2}\left\|\partial_{y}^{2} v\right\|_{L^{\infty}} .
$$

The decomposition of $v$ implies that

$$
\partial_{y} v=\frac{b y}{\sqrt{2-2 a} \sqrt{2+b y^{2}}}+\beta_{0}+\beta_{3} \cos \theta+\beta_{4} \sin \theta+\partial_{y}\left\{e^{\frac{a y^{2}}{2}} \xi\right\}
$$

Hence we get

$$
\left\|\langle y\rangle^{-1} \partial_{y} v\right\|_{L^{\infty}} \leq b+\left|\beta_{0}\right|+\left|\beta_{3}\right|+\left|\beta_{4}\right|+\left\|\langle y\rangle^{-1} \partial_{y}\left[e^{\frac{a y^{2}}{2}} \xi\right]\right\|_{L^{\infty}} \lesssim \beta .
$$

Recall that $\left\|\langle y\rangle^{-1} \partial_{y}\left[e^{\frac{a y^{2}}{2}} \xi\right]\right\|_{L^{\infty}}=\left\|e^{\frac{a y^{2}}{2}} \xi\right\|_{1,1}$, and that by assumption on $M_{1,1}$ we have $\left\|e^{\frac{a y^{2}}{4}} \xi\right\|_{1,1} \lesssim \beta^{\frac{21}{20}}$. This, together with the estimate $\left|\partial_{y}^{2} v\right|=\mathcal{O}\left(\beta^{\frac{13}{20}}\right)$ in (6.8) implies that

$$
\left|A_{4}\right| \lesssim \beta^{\frac{53}{20}} .
$$

We use the definition of the quantity $N_{3,2}$ introduced in (7.6) to decompose $A_{5}$ into four terms,

$$
\begin{aligned}
& A_{5}=\langle\left.y e^{-\frac{a y^{2}}{4}},\left[v^{-2}-V_{a, b}^{-2}\right] \partial_{\theta}^{2} \xi e^{-\frac{a y^{2}}{4}}\right\rangle \\
&-\left\langle y e^{-\frac{a y^{2}}{4}}, v^{-2} \frac{q^{2}}{1+p^{2}+q^{2}} \partial_{\theta}^{2} \xi e^{-\frac{a y^{2}}{4}}\right\rangle \\
&+\left\langle y e^{-\frac{a y^{2}}{4}}, v^{-1} \frac{2 p q}{1+p^{2}+q^{2}} \partial_{\theta} \partial_{y} v e^{-\frac{a y^{2}}{4}}\right\rangle \\
&+\left\langle y e^{-\frac{a y^{2}}{4}}, v^{-2} \frac{q}{1+p^{2}+q^{2}} \partial_{\theta} v e^{-\frac{a y^{2}}{4}}\right\rangle \\
&=\sum_{\ell=1}^{4} A_{5, \ell},
\end{aligned}
$$

where the various terms $A_{5, \ell}(\ell=1, \ldots, 4)$ are naturally defined.

For $A_{5,1}$, we integrate by parts in $\theta$ to remove the slowly decaying $\theta$-independent components in $v^{-2}-V_{a, b}^{-2}$. Thus we compute that

$$
A_{5,1}=2\left\langle y e^{-\frac{a y^{2}}{2}}, v^{-3} \partial_{\theta} v \partial_{\theta} \xi\right\rangle
$$

and hence can estimate

$$
\left|A_{5,1}\right| \lesssim\left\|v^{-2} \partial_{\theta} v\right\|_{L^{\infty}}\left\|\langle y\rangle^{-3} e^{\frac{a y^{2}}{4}} \partial_{\theta} \xi\right\|_{L^{\infty}} .
$$


We relate $e^{\frac{a y^{2}}{4}} \partial_{\theta} \xi$ to $\partial_{\theta} v$ using the decomposition of $v$ in (6.1), obtaining

$$
\begin{aligned}
\langle y\rangle^{-2} e^{\frac{a y^{2}}{4}}\left|\partial_{\theta} \xi\right| & \leq\langle y\rangle^{-2}\left|\partial_{\theta} v\right|+\sum_{k=1}^{4}\left|\beta_{k}\right| \\
& \lesssim v^{-2}\left|\partial_{\theta} v\right|+\sum_{k=1}^{4}\left|\beta_{k}\right| \\
& \lesssim \beta^{\frac{33}{20}}
\end{aligned}
$$

Here we used the estimate $v^{-2}\left|\partial_{\theta} v\right| \lesssim \beta^{\frac{33}{20}}$ from (6.9) along with the fact that

$$
\langle y\rangle^{-1} \lesssim v^{-1}, \quad \text { equivalently, } v \lesssim\langle y\rangle .
$$

This, in turn, is implied by three facts: the computation that

$$
|v(y, \theta, \tau)| \leq|v(0, \theta, \tau)|+\int_{0}^{y}\left|\partial_{z} v(z, \theta, \tau)\right| \mathrm{d} z,
$$

the consequence $v(0, \theta, \tau) \lesssim 1$ of our input assumption that $M_{3,0} \lesssim \beta^{-\frac{1}{20}}$, and the assumption that $\left|\partial_{y} v\right| \lesssim 1$ from (6.6). We collect the estimates above to obtain

$$
\left|A_{5,1}\right| \lesssim \beta^{\frac{33}{10}}
$$

Turning to $\sum_{\ell=2}^{4} A_{5, \ell}$, we observe that each of these terms contains a rapidly decaying factor $q=v^{-1} \partial_{\theta} v$. We apply (A.11) again to get

$$
\left\|\langle y\rangle^{-1} q\right\|_{L^{\infty}} \lesssim\left\|v^{-2} \partial_{\theta} v\right\|_{L^{\infty}} \lesssim \beta^{\frac{33}{20}} .
$$

This, together with the estimates in (6.9), implies that

$$
\left|\sum_{\ell=2}^{4} A_{5, \ell}\right| \lesssim \beta^{\frac{16}{5}} .
$$

Collecting the estimates above completes the proof of estimate (7.10).

Proof of estimate (7.13). Let $\tau_{\#}$ denote $\tau\left(t_{\#}\right)$, and recall the boundary condition $\beta_{0}\left(\tau_{\#}\right)=0$ from (7.3). We use (7.10) to write

$$
\left|\beta_{0}(\tau)\right| \lesssim \int_{\tau}^{\tau \# \#} e^{-\int_{\kappa}^{\tau} \# \Omega(a, b)(s) \mathrm{d} s} \beta^{\frac{13}{5}}(\kappa) \mathrm{d} \kappa
$$

Then we use the consequence of (7.7) that $\Omega(a, b) \geq 1 / 2$ to conclude that

$$
\left|\beta_{0}(\tau)\right| \lesssim \beta^{\frac{13}{5}}(\tau)
$$

\section{A.4. Proofs of estimates (7.11) and (7.14).}

Proof of estimate (7.11). As we did in deriving (A.6), we take the inner product of (7.4) with $\cos \theta e^{-\frac{a y^{2}}{4}}$ to obtain

$$
\left\langle\cos \theta e^{-\frac{a y^{2}}{4}}, \partial_{\tau} \xi\right\rangle=\sum_{k=1}^{5} \tilde{A}_{k},
$$

where the terms $\tilde{A}_{k}$ are defined like those in (A.6), replacing $y$ by $\cos \theta$ where needed. 
The estimates for the various terms are very similar to those in the proof of estimate (7.10). The only difference is that the presence of the factor $\cos \theta$ here allows us to integrate by parts in $\theta$ to remove some slowly-decaying $\theta$-independent parts. In what follows, we estimate $\tilde{A}_{1}, \tilde{A}_{2}$, and $\tilde{A}_{4}$ in detail to illustrate the main ideas. We omit detailed proofs of the estimates for the other terms.

For $\tilde{A}_{1}$, we have

$$
\begin{aligned}
\tilde{A}_{1} & =-\left\langle\cos \theta e^{-\frac{a y^{2}}{4}},\left[-\partial_{y}^{2}+\frac{a^{2}+a_{\tau}}{4} y^{2}-\frac{3 a}{2}-V_{a, b}^{-2} \partial_{\theta}^{2}-V_{a, b}^{-2}\right] \xi\right\rangle \\
& =-\frac{a_{\tau}}{4}\left\langle\cos \theta y^{2} e^{-\frac{a y^{2}}{4}}, \xi\right\rangle \\
& =\frac{a_{\tau}}{4}\left\langle\sin \theta y^{2} e^{-\frac{a y^{2}}{4}}, \partial_{\theta} \xi\right\rangle .
\end{aligned}
$$

In the second step, we used the simple observation $\left[V_{a, b}^{-2} \partial_{\theta}^{2}+V_{a, b}^{-2}\right] \cos \theta=0$; and in the last step, we integrated by parts in $\theta$. This calculation directly implies that

$$
\begin{aligned}
\left|\tilde{A}_{1}\right| & \lesssim\left|a_{\tau}\right| \min \left\{\left\|\langle y\rangle^{-3} e^{\frac{a y^{2}}{4}} \xi\right\|_{L^{\infty}},\left\|\langle y\rangle^{-5} e^{\frac{a y^{2}}{4}} \partial_{\theta} \xi\right\|_{L^{\infty}}\right\} \\
& \lesssim \min \left\{\beta^{\frac{7}{2}}, \beta^{2}\left\|\langle y\rangle^{-5} e^{\frac{a y^{2}}{4}} \partial_{\theta} \xi\right\|_{L^{\infty}}\right\} .
\end{aligned}
$$

For $\tilde{A}_{2}$, one can use symmetries to cancel many terms, as we did in Section A.3 in the proof of estimate (7.10). Here we get

$$
\begin{aligned}
\tilde{A}_{2} & =\left\langle\cos \theta e^{-\frac{a y^{2}}{2}},\left[\partial_{y}^{2}+V_{a, b}^{-2} \partial_{\theta}^{2}-a y \partial_{y}+a+V_{a, b}^{-2}-\partial_{\tau}\right] \beta_{1} \cos \theta\right\rangle \\
& =\left[a \beta_{1}-\partial_{\tau} \beta_{1}\right] \int_{0}^{2 \pi} \int_{-\infty}^{\infty} \cos ^{2} \theta e^{-\frac{a y^{2}}{2}} \mathrm{~d} y \mathrm{~d} \theta .
\end{aligned}
$$

For $\tilde{A}_{4}$, we integrate by parts in $\theta$ to remove the $\theta$-independent components, yielding

$$
\tilde{A}_{4}:=\left\langle\cos \theta e^{-\frac{a y^{2}}{4}}, N_{2}\right\rangle=-\left\langle\sin \theta e^{-\frac{a y^{2}}{4}}, \partial_{\theta} N_{2}\right\rangle .
$$

Using the definition of $N_{2}$, this becomes

$$
\begin{aligned}
\tilde{A}_{4}=-\left\langle\sin \theta e^{-\frac{a y^{2}}{4}},\right. & e^{-\frac{a y^{2}}{4}} \frac{2 p \partial_{\theta} p}{1+p^{2}+q^{2}} \partial_{y}^{2} v-e^{-\frac{a y^{2}}{4}} \frac{2 p^{2}\left[p \partial_{\theta} p+q \partial_{\theta} q\right]}{\left(1+p^{2}+q^{2}\right)^{2}} \partial_{y}^{2} v \\
& \left.+e^{-\frac{a y^{2}}{4}} \frac{p^{2}}{1+p^{2}+q^{2}} \partial_{y}^{2} \partial_{\theta} v\right\rangle \\
= & K_{1}+K_{2}+K_{3},
\end{aligned}
$$

where the terms $K_{\ell}(\ell=1,2,3)$ are naturally defined.

It is easy to estimate $K_{2}$ and $K_{3}$. Indeed, using the estimates in (6.9) and the estimate $\langle y\rangle^{-1} \lesssim v^{-1}$ from (A.11), we get

$$
\left|K_{\ell}\right| \lesssim b^{\frac{18}{5}}
$$

for $\ell=2,3$. Estimating $K_{1}$ is more involved. We begin by integrating by parts in the $y$ variable, using $2 p \partial_{y}^{2} v=\partial_{y}\left(\partial_{y} v\right)^{2}$ to obtain

$$
K_{1}=\left\langle\partial_{y}\left[\sin \theta e^{-\frac{a y^{2}}{2}} \frac{\partial_{\theta} \partial_{y} v}{1+p^{2}+q^{2}}\right],\left(\partial_{y} v\right)^{2}\right\rangle .
$$


We bound the term $\langle y\rangle^{-2}\left(\partial_{y} v\right)^{2}$ by $\beta^{2}$ as in (A.8). Then we use (6.9) to see that the terms in $\partial_{y}\left\{\sin \theta e^{-\frac{a y^{2}}{2}} \frac{\partial_{\theta} \partial_{y} v}{1+p^{2}+q^{2}}\right\}$ are of order $\beta^{\frac{8}{5}}$. Thus we get

$$
\left|K_{1}\right| \lesssim \beta^{\frac{18}{5}}
$$

We collect the estimates obtained above to conclude that

$$
\left|\tilde{A}_{4}\right| \lesssim \beta^{\frac{18}{5}}
$$

For $\tilde{A}_{3}$ and $\tilde{A}_{5}$, we apply similar methods to conclude that

$$
\left|\tilde{A}_{3}\right|+\left|\tilde{A}_{5}\right| \lesssim \min \left\{\beta^{\frac{16}{5}}, \beta^{\frac{31}{20}}\left\|\langle y\rangle^{-5} e^{\frac{a y^{2}}{4}} \partial_{\theta} \xi\right\|_{L^{\infty}}\right\} .
$$

This completes our proof of estimate (7.11).

Proof of estimate (7.14). By the boundary condition (7.3), we have $\beta_{1}\left(\tau_{\#}\right)=0$, where we again define $\tau_{\#}=\tau\left(t_{\#}\right)$. We rewrite estimate (7.11) in the form

$$
\left|\partial_{\tau} \beta_{1}-a \beta_{1}\right| \lesssim \min \left\{\beta^{\frac{16}{5}},\left(M_{4}+1\right) \beta^{\frac{18}{5}}\right\} .
$$

This implies that

$$
\left|\beta_{1}(\tau)\right| \lesssim \int_{\tau}^{\tau \#} e^{-\int_{\kappa}^{\tau} \# a(s) \mathrm{d} s} \cdot \min \left\{\beta^{\frac{16}{5}},\left(M_{4}+1\right) \beta^{\frac{18}{5}}\right\}(\kappa) \mathrm{d} \kappa .
$$

Using the fact that $a \geq 1 / 4$, we thus conclude that

$$
\left|\beta_{1}(\tau)\right| \lesssim \min \left\{\beta^{\frac{16}{5}}(\tau),\left(M_{4}(\tau)+1\right) \beta^{\frac{18}{5}}(\tau)\right\} .
$$

\section{Appendix B. Proof of Lemma 11}

Proof of Lemma 11. By differentiating equation (7.4), we find that $\partial_{\theta} \xi$ evolves by

$$
\partial_{\tau}\left(\partial_{\theta} \xi\right)=-L_{0}(a)\left[\partial_{\theta} \xi\right]+\sum_{k=1}^{3} D_{k},
$$

where the terms on the RHS are defined by

$$
\begin{aligned}
L_{0}(a) & :=-\partial_{y}^{2}+\frac{a^{2}+\partial_{\tau} a}{4} y^{2}-\frac{a}{2}-1-\frac{1}{2} \partial_{\theta}^{2}, \\
D_{1} & :=\left[1-a-\frac{2-2 a}{2+b y^{2}}\right] \partial_{\theta} \xi, \\
D_{2} & :=\partial_{\theta} F(a, b, \beta), \\
D_{3} & :=\sum_{\ell=1}^{3} \partial_{\theta} N_{\ell} .
\end{aligned}
$$

Recall that the nonlinear terms $N_{\ell}$ appearing above are defined in Section 7.

Our first observations are the following key facts.

Lemma 14. For all times that the assumptions in Section 6.1 hold, one has the orthogonality condition

$$
e^{-\frac{a}{4} y^{2}} \partial_{\theta} \xi \perp\left\{1, y, y^{2}, \cos \theta, \sin \theta, y \cos \theta, y \sin \theta\right\},
$$


along with estimates

$$
\sum_{k=1}^{3}\left\|\langle y\rangle^{-5} e^{\frac{a}{4} y^{2}} D_{k}\right\|_{L^{\infty}} \lesssim \beta^{\frac{13}{5}} .
$$

This lemma is proved in Section B.1 below.

In what follows, we pursue the strategies outlined in Section 7. Our next step is to put (B.1) into a more convenient form by removing the time dependency of the linear operator $L_{0}(a)$, which is what makes it difficult to estimate the propagator. The parametrization that follows is identical to that used in [13].

Recall that $\tau(t):=\int_{0}^{t} \lambda^{-2}(s) \mathrm{d} s$ for any $\tau \geq 0$, and $a(\tau):=-\lambda(t(\tau)) \partial_{t} \lambda(t(\tau))$. Let $t(\tau)$ be the inverse function of $\tau(t)$, and fix a ( $\tau$-timescale) constant $T_{1}>0$. We approximate $\lambda(t(\tau))$ on the interval $0 \leq \tau \leq T_{1}$ by a new gauge $\lambda_{1}(t(\tau))$, chosen so that $\lambda_{1}\left(t\left(T_{1}\right)\right)=\lambda\left(t\left(T_{1}\right)\right)$, and $\alpha:=-\lambda_{1}(t(\tau)) \partial_{t} \lambda_{1}(t(\tau))=a\left(T_{1}\right)$ is constant.

Then we introduce a new spatial variable $z(x, t):=\lambda_{1}^{-1}(t) x$ and a new time variable $\sigma(t):=\int_{0}^{t} \lambda_{1}^{-2}(s) \mathrm{d} s$, together with a new function $\eta(z, \theta, \sigma)$ defined by

$$
\lambda_{1}(t) e^{\frac{\alpha}{4} z^{2}} \eta(z, \theta, \sigma):=\lambda(t) e^{\frac{a(\tau)}{4} y^{2}} \xi(y, \theta, \tau) \equiv \lambda(t) \phi(y, \theta, \tau) .
$$

One should keep in mind that the variables $z$ and $y$ are related by $z / y=\lambda(t) / \lambda_{1}(t)$. To relate the time scales $\sigma(t):=\int_{0}^{t} \lambda_{1}^{-2}(s) \mathrm{d} s$ and $\tau=\int_{0}^{t} \lambda^{-2}(s) \mathrm{d} s$, we note that one may for all $t(\tau)<T_{1}$ regard $\sigma$ as a function of $\tau$ given by

$$
\sigma(\tau):=\int_{0}^{t(\tau)} \lambda_{1}^{-2}(s) \mathrm{d} s
$$

Observing that the function $\sigma$ is invertible, we denote its inverse by $\tau(\sigma)$.

Now we derive an equation for $\partial_{\theta} \eta$, which is related to $\partial_{\theta} \xi$ by (B.4). One has

$$
\partial_{\sigma}\left[\partial_{\theta} \eta(\sigma)\right]=-L_{0}(\alpha)\left[\partial_{\theta} \eta(\sigma)\right]+\tilde{D}_{1}+\tilde{D}_{2}+\tilde{D}_{3},
$$

where the operator $L_{0}(\alpha)$ is linear and autonomous,

$$
L_{0}(\alpha):=-\partial_{z}^{2}+\frac{\alpha^{2}}{4} z^{2}-\frac{\alpha}{2}-1-\frac{1}{2} \partial_{\theta}^{2} .
$$

The remaing terms on the RHS are

$$
\begin{aligned}
& \tilde{D}_{1}:=\left[-\frac{\lambda^{2}}{\lambda_{1}^{2}} \frac{2-2 a}{2+b(\tau(\sigma)) y^{2}}+1-\alpha\right] \partial_{\theta} \eta, \\
& \tilde{D}_{2}:=\frac{\lambda_{1}}{\lambda} e^{-\frac{\alpha}{4} z^{2}} e^{\frac{a}{4} y^{2}} D_{2}, \\
& \tilde{D}_{3}:=\frac{\lambda_{1}}{\lambda} e^{-\frac{\alpha}{4} z^{2}} e^{\frac{a}{4} y^{2}} D_{3} .
\end{aligned}
$$

We estimate those terms in the following.

Lemma 15. If the assumptions of Section 6.1 hold, then there exists a constant $C$ such that one has

$$
\begin{gathered}
\frac{\tau}{C} \leq \sigma(\tau) \leq C \tau \\
|a-\alpha|+\left|\frac{\lambda_{1}(t)}{\lambda(t)}-1\right| \leq C \beta(\tau(t)),
\end{gathered}
$$


and

$$
\frac{1}{C} \leq \frac{\langle y\rangle}{\langle z\rangle} \leq C
$$

in the time interval $\tau \in\left[0, T_{1}\right]$. Moreover,

$$
\sum_{k=1}^{3}\left\|\langle z\rangle^{-5} e^{\frac{\alpha}{4} z^{2}} \tilde{D}_{k}\right\|_{L^{\infty}} \lesssim \beta^{\frac{13}{5}}(\tau(\sigma))
$$

This lemma is proved in Section B.2.

Now we apply Duhamel's principle to the evolution equation (B.6), obtaining

$$
\partial_{\theta}\left[\eta\left(\sigma\left(T_{1}\right)\right)\right]=e^{-\sigma\left(T_{1}\right) L_{0}(\alpha)} \partial_{\theta} \eta_{0}+\int_{0}^{\sigma\left(T_{1}\right)} e^{-\left(\sigma\left(T_{1}\right)-\sigma_{1}\right) L_{0}(\alpha)} \sum_{k=1}^{3} \tilde{D}_{k}\left(\sigma_{1}\right) \mathrm{d} \sigma_{1} .
$$

The definition of $\eta$ implies that at time $t=T_{1}$, one has $y=z, \alpha=a\left(\tau\left(T_{1}\right)\right)$ and $\eta=\xi$. Together with the orthogonality conditions for $\partial_{\theta} \xi$ in (B.2), this implies that

$$
\begin{aligned}
\partial_{\theta} \eta\left(\sigma\left(T_{1}\right)\right) & =\mathbb{P}_{7}\left[\partial_{\theta} \eta\left(\sigma\left(T_{1}\right)\right)\right] \\
& =\mathbb{P}_{7}\left[e^{-\sigma L(\alpha)} \partial_{\theta} \eta_{0}\right]+\int_{0}^{\sigma\left(T_{1}\right)} e^{-\left(\sigma\left(T_{1}\right)-\sigma_{1}\right) L_{0}(\alpha)} \mathbb{P}_{7}\left\{\sum_{k=1}^{3} \tilde{D}_{k}\left(\sigma_{1}\right)\right\} \mathrm{d} \sigma_{1}
\end{aligned}
$$

where $\mathbb{P}_{7}$ denotes orthogonal projection onto the subspace orthogonal to the seven functions in (B.2).

To prove that $\eta$ is sufficiently small, we use the following propagator estimates.

Lemma 16. Let the assumptions of Section 6.1 hold, Then for any time $\sigma \geq 0$, one has

$$
\left\|\langle z\rangle^{-5} e^{\frac{\alpha z^{2}}{4}} e^{-\sigma L(\alpha)} \mathbb{P}_{7}[\eta]\right\|_{L^{\infty}} \lesssim e^{-\alpha \sigma}\left\|\langle z\rangle^{-5} e^{\frac{\alpha z^{2}}{4}} \eta\right\|_{L^{\infty}} .
$$

This lemma is proved in Section B.3 below.

Now we use estimate (B.10) to obtain

$$
\begin{aligned}
\left\|\langle z\rangle^{-5} e^{\frac{\alpha z^{2}}{4}} \partial_{\theta} \eta\left(\sigma\left(T_{1}\right)\right)\right\|_{L^{\infty}} & \lesssim e^{-\alpha \sigma}\left\|\langle z\rangle^{-5} e^{\frac{\alpha z^{2}}{4}} \partial_{\theta} \eta(0)\right\|_{L^{\infty}} \\
& \quad+\int_{0}^{\sigma\left(T_{1}\right)} e^{-\alpha \sigma}\left\|\langle z\rangle^{-5} e^{\frac{\alpha z^{2}}{4}} \sum_{k=1}^{3} \tilde{D}_{k}\right\|_{L^{\infty}} \mathrm{d} \sigma \\
& \lesssim e^{-\alpha \sigma} \beta^{\frac{21}{10}}(0)+\int_{0}^{\sigma\left(T_{1}\right)} e^{-\alpha \sigma} \beta^{\frac{13}{5}}(\tau(\sigma)) \mathrm{d} \sigma \\
& \lesssim \beta^{\frac{21}{10}}\left(\sigma\left(T_{1}\right)\right) .
\end{aligned}
$$

Note that here we used the new condition $\left\|\langle x\rangle^{-5} \partial_{\theta} u_{0}\right\|_{L^{\infty}} \lesssim b^{\frac{21}{10}}(0)$ implied by Assumption [A1] to get $\left\|\langle y\rangle^{-5} e^{\frac{a(0) y^{2}}{4}} \partial_{\theta} \xi(0)\right\|_{L^{\infty}} \lesssim \beta^{\frac{21}{10}}(0)$. Finally, we complete the proof by observing that

$$
\langle z\rangle^{-5} e^{\frac{\alpha z^{2}}{4}} \partial_{\theta} \eta\left(\sigma\left(T_{1}\right)\right)=\langle y\rangle^{-5} e^{\frac{a y^{2}}{4}} \partial_{\theta} \xi\left(T_{1}\right) .
$$

The desired estimate follows, and the proof of Lemma 11 is complete. 


\section{B.1. Proof of Lemma 14.}

Proof of Lemma 14. We start by proving the orthogonality condition. By (7.2), we see that $\xi$ is orthogonal to seven functions. By a simple integration by parts in $\theta$, we find that $\partial_{\theta} \xi$ is also orthogonal to these same functions, which proves (B.2).

Next we turn to (B.3). Three observations make it relatively straightforward to estimate the various terms there: (I) Most of the terms are of higher orders in $\xi, \partial_{y} v, \partial_{\theta} v$, and $\partial_{y} \partial_{\theta} v$, which (appropriately weighted) are small in $L^{\infty}$. (II) The presence of the operator $\partial_{\theta}$ removes the slowly-changing $\theta$-independent terms. And (III), the high weight $\langle y\rangle^{-5}$ here allows us to be generous when doing estimates.

We begin with the term $D_{2}=\partial_{\theta} F=\partial_{\theta}\left[F_{1}(a, b)+F_{2}(a, b, \beta)\right]$. The fact that $F_{1}$ is independent of $\theta$ makes $\partial_{\theta} F=\partial_{\theta} F_{2}$. Moreover the $\beta_{0}$-component, which is also $\theta$-independent, will vanish after applying $\partial_{\theta}$. Together with the estimates $\partial_{\tau} \beta_{k}-a \beta_{k}=\mathcal{O}\left(\beta^{\frac{16}{5}}\right), k=1,2$, and $\partial_{\tau} \beta_{\ell}=\mathcal{O}\left(\beta^{\frac{16}{5}}\right), \ell=3,4$, from (7.11) of Lemma 9, this implies that

$$
\left\|\langle y\rangle^{-5} e^{\frac{a y^{2}}{4}} D_{2}\right\|_{L^{\infty}} \lesssim \beta^{3}
$$

For $D_{1}$, we relate $e^{\frac{a y^{2}}{4}} \xi$ to $v$ using (6.1), and we bound $\langle y\rangle^{-2}\left(\frac{\frac{1}{2}+a}{2}-\frac{\frac{1}{2}+a}{2+b y^{2}}\right)$ by $\beta$, obtaining

$$
\left\|\langle y\rangle^{-5} e^{\frac{a y^{2}}{4}} D_{1}\right\|_{L^{\infty}} \lesssim \beta\left\{\left\|v^{-2} \partial_{\theta} v\right\|_{L^{\infty}}+\sum_{k=1}^{4}\left|\beta_{k}\right|\right\} \lesssim \beta^{\frac{5}{2}} .
$$

Here, we used the estimate $\langle y\rangle^{-1} \lesssim v^{-1}$ from (A.11) and an assumption on $v^{-2}\left|\partial_{\theta} v\right|$ from the outputs of the first bootstrap machine in Section 4.2.

We decompose $D_{5}$ as

$$
\begin{aligned}
\left|\langle y\rangle^{-5} \partial_{\theta} N_{3} e^{\frac{a y^{2}}{4}}\right| \lesssim & \langle y\rangle^{-2}\left|V_{a, b}^{-2}-\frac{1}{2}\right|\left[v^{-3}\left|\partial_{\theta}^{3} v\right|+\sum_{k=1}^{k}\left|\beta_{k}\right|\right] \\
& +\langle y\rangle^{-3}\left|v^{-2}-V_{a, b}^{-2}\right|\left[v^{-2}\left|\partial_{\theta}^{3} v\right|+\sum_{k=1}^{k}\left|\beta_{k}\right|\right] \\
& +\langle y\rangle^{-3}\left|\partial_{\theta}\left[v^{-2} \frac{1+p^{2}}{1+p^{2}+q^{2}}\right]\right| v^{-2}\left|\partial_{\theta}^{2} v\right| \\
& +\langle y\rangle^{-5}\left|\partial_{\theta}\left[v^{-1} \frac{2 p q}{1+p^{2}+q^{2}} \partial_{\theta} \partial_{y} v\right]\right| \\
& +\langle y\rangle^{-5}\left|\partial_{\theta}\left[v^{-2} \frac{q}{1+p^{2}+q^{2}} \partial_{\theta} v\right]\right| \\
= & \sum_{l=1}^{5} K_{\ell}
\end{aligned}
$$

with $K_{\ell}(\ell=1, \ldots, 5)$ naturally defined.

For $K_{1}$, we use the assumption $A \lesssim \beta^{-\frac{1}{20}}$ for the quantity $A$ defined in (6.3) to estimate the factor

$$
\langle y\rangle^{-2}\left|V_{a, b}^{-2}-\frac{1}{2}\right| \lesssim\langle y\rangle^{-2}\left(\left|\frac{1}{2}-a\right|+b y^{2}\right) \lesssim \beta .
$$


By (6.9), the quantity $v^{-2}\left|\partial_{\theta}^{3} v\right|$ is bounded by $\beta^{\frac{33}{20}}$. Combining these estimates with the consequence $\left|\beta_{k}\right| \lesssim \beta^{2}$ of Lemma 9 implies that

$$
\left|K_{1}\right| \lesssim \beta^{\frac{13}{5}}
$$

For $K_{2}$, we use ideas similar to those we used to control $K_{1}$ to obtain

$$
\left\|\langle y\rangle^{-5} e^{\frac{a y^{2}}{4}} K_{2}\right\|_{L^{\infty}} \lesssim \beta^{\frac{16}{5}}
$$

The presence of factors $q=v^{-1} \partial_{\theta} v$ and $\partial_{\theta} \partial_{y} v$ makes it relatively easy to control the terms in $K_{3}$ and $K_{4}$. Indeed, one may apply the estimates in (6.9) to obtain

$$
\left\|\langle y\rangle^{-5} e^{\frac{a y^{2}}{4}}\left[K_{3}+K_{4}\right]\right\|_{L^{\infty}} \lesssim \beta^{3} .
$$

The claimed estimate for $D_{5}$ follows when one combines the estimates above.

\section{B.2. Proof of Lemma 15.}

Proof of Lemma 15. Because the first three estimates are obtained exactly as in the corresponding part of [13], we do not repeat those arguments here.

For (B.10), we begin by decomposing $\tilde{D}_{1}$ into three terms,

$\tilde{D}_{1}=\left[1-\frac{\lambda^{2}}{\lambda_{1}^{2}}\right] \frac{2-2 a}{2+b(\tau(\sigma)) y^{2}} \partial_{\theta} \eta+\frac{2 a-2 \alpha}{2+b(\tau(\sigma)) y^{2}} \partial_{\theta} \eta+(2-2 \alpha) \frac{b(\tau(\sigma)) y^{2}}{2\left(2+b(\tau(\sigma)) y^{2}\right)} \partial_{\theta} \eta$.

The factor $b y^{2}$ in the last term on the RHs is controlled by $\langle y\rangle^{-2} b y^{2} \leq b \lesssim \beta$, which together with the estimates for $a-\alpha$ and $\lambda / \lambda_{1}-1$ in (B.8) implies that

$$
\left\|\langle y\rangle^{-5} e^{\frac{\alpha z^{2}}{4}} \tilde{D}_{1}\right\|_{L^{\infty}} \lesssim b\left\|\langle z\rangle^{-3} e^{\frac{\alpha}{4} z^{2}} \partial_{\theta} \eta(\sigma)\right\|_{L^{\infty}} \lesssim \beta\left\|\langle y\rangle^{-3} e^{\frac{a}{4} y^{2}} \partial_{\theta} \xi(\tau(\sigma))\right\|_{L^{\infty}} .
$$

Note that in the last step, we used (B.9) and the definition of $\eta$. Using (A.10), which relates $\partial_{\theta} \xi$ to $\partial_{\theta} v$, we get

$$
\left\|\langle y\rangle^{-5} e^{\frac{\alpha z^{2}}{4}} \tilde{D}_{1}\right\|_{L^{\infty}} \lesssim \beta^{\frac{51}{20}}
$$

We relate the quantities $\tilde{D}_{2}$ and $\tilde{D}_{3}$ to $D_{2}$ and $D_{3}$, respectively, using (B.8). The we apply estimate (B.3) to obtain

$$
\begin{aligned}
\left\|\langle z\rangle^{-5} e^{\frac{\alpha}{4} z^{2}} \tilde{D}_{k}(\sigma)\right\|_{L^{\infty}} & \lesssim\left\|\langle y\rangle^{-5} e^{\frac{a}{4} y^{2}} D_{k}(\tau(\sigma))\right\|_{L^{\infty}} \\
& \lesssim \beta^{\frac{13}{5}}(\tau(\sigma)) .
\end{aligned}
$$

The proof is complete.

\section{B.3. Proof of Lemma 16.}

Proof of Lemma 16. In our earlier work [13], we proved a similar result but with the weight $\langle z\rangle^{-3}$. To prove an estimate with the weight $\langle z\rangle^{-5}$, we slightly generalize a result proved in [12], which in turn is motivated by a result from [6].

In [12], the functions are $\theta$-independent. So before adapting the arguments there, we decompose $\eta$ as

$$
\eta(z, \theta)=\sum_{k=-\infty}^{\infty} e^{i k \theta} f_{k}(z)
$$


with $f_{k}(z):=\frac{1}{2 \pi} \int_{\mathbb{S}^{1}} e^{-i k \theta} \eta(z, \theta) \mathrm{d} \theta$. The explicit form of $\mathbb{P}_{7}$ allows us to write

$$
\mathbb{P}_{7}[\eta]=\mathbb{P}_{3}\left[f_{0}\right](z)+e^{i \theta} \mathbb{P}_{2}\left[f_{1}\right](z)+e^{-i \theta} \mathbb{P}_{2}\left[f_{-1}\right](z)+\sum_{|k|>2} e^{i k \theta} f_{k}(z)
$$

and

$$
\begin{aligned}
e^{-\sigma L(\alpha)} \mathbb{P}_{7}[\eta]= & e^{-\sigma L_{0}(\alpha)} \mathbb{P}_{3}\left[f_{0}\right](z)+e^{i \theta} e^{-\sigma\left[L_{0}(\alpha)+\frac{1}{2}\right]} \mathbb{P}_{2}\left[f_{1}\right](z) \\
& +e^{-i \theta} e^{-\sigma\left[L_{0}(\alpha)+\frac{1}{2}\right]} \mathbb{P}_{2}\left[f_{-1}\right](z)+\sum_{|k|>2} e^{i k \theta} e^{-\sigma\left[L_{0}(\alpha)+\frac{k^{2}}{2}\right]} f_{k}(z) .
\end{aligned}
$$

Here, $\mathbb{P}_{3}$ denotes orthogonal projection onto the subspace orthogonal to $e^{-\frac{\alpha z^{2}}{4}}$, $z e^{-\frac{\alpha z^{2}}{4}}$, and $\left(\alpha z^{2}-1\right) e^{-\frac{\alpha z^{2}}{4}}$, while $\mathbb{P}_{2}$ denotes orthogonal projection onto the subspace orthogonal to $e^{-\frac{\alpha z^{2}}{4}}$ and $z e^{-\frac{\alpha z^{2}}{4}}$.

In what follows, we focus on the first term on the RHS above; the remaining terms are estimated similarly. To generalize Lemma 17 from [12], we note that in the present situation, one has $\beta=0$ and $V=0$, which will make our analysis easier.

Following [12], we derive an integral kernel for $e^{-\sigma L_{0}(\alpha)}$ such that

$$
e^{-\sigma L_{0}(\alpha)} \mathbb{P}_{3}\left[f_{0}\right](z)=e^{\frac{\alpha}{4}|z|^{2}} \int_{-\infty}^{\infty} U_{0}(z, y) f(y) \mathrm{d} y
$$

where

$$
f(y):=e^{-\frac{\alpha}{4}|y|^{2}} \mathbb{P}_{3}\left[f_{0}\right](y),
$$

and the integral kernel $U_{0}$ is

$$
U_{0}(z, y):=4 \pi\left(1-e^{-2 \alpha \sigma}\right)^{-\frac{1}{2}} \sqrt{\alpha} e^{2 \alpha \sigma} e^{-\frac{\alpha\left(z-e^{-\alpha \sigma} y\right)^{2}}{2\left(1-e^{-2 \alpha \sigma}\right)}} .
$$

To obtain the decay estimate, we integrate by parts in $y$ three times, exploiting the fact that $\partial_{y} U_{0}(z, y)$ has a factor $e^{-\alpha \sigma}$. To prepare for this, namely to ensure that the functions we consider are in appropriate spaces, we use the fact that $\mathbb{P}_{3}\left[f_{0}\right] \perp\left\{e^{-\frac{\alpha}{4}|z|^{2}}, z e^{-\frac{\alpha}{4}|z|^{2}}, z^{2} e^{-\frac{\alpha}{4}|z|^{2}}\right\}$ to see that $f \perp\left\{1, z, z^{2}\right\}$, hence that

$$
\begin{gathered}
\int_{-\infty}^{\infty} f(y) \mathrm{d} y=0 \\
\int_{-\infty}^{\infty} \int_{-\infty}^{y} f\left(y_{1}\right) \mathrm{d} y_{1} \mathrm{~d} y=\int_{-\infty}^{\infty} y f(y) \mathrm{d} y=0, \\
\int_{-\infty}^{\infty} \int_{-\infty}^{y} \int_{-\infty}^{y_{1}} f\left(y_{2}\right) \mathrm{d} y_{2} \mathrm{~d} y_{1} \mathrm{~d} y=\int_{-\infty}^{\infty} y^{2} f(y) \mathrm{d} y=0 .
\end{gathered}
$$

Furthermore, there exists a constant $C$, independent of $y$, such that

$$
\left|f^{(-3)}(y)\right| \leq C e^{-\frac{\alpha y^{2}}{2}}\left(1+|y|^{2}\right)\left\|\langle z\rangle^{-5} e^{\frac{\alpha z^{2}}{4}} f_{0}\right\|_{L^{\infty}}
$$

where

$$
f^{(-3)}(y):=\int_{-\infty}^{y} \int_{-\infty}^{y_{1}} \int_{-\infty}^{y_{2}} f\left(y_{3}\right) \mathrm{d} y_{3} \mathrm{~d} y_{2} \mathrm{~d} y_{1}=-\int_{y}^{\infty} \int_{y_{1}}^{\infty} \int_{y_{2}}^{\infty} f\left(y_{3}\right) \mathrm{d} y_{3} \mathrm{~d} y_{2} \mathrm{~d} y_{1} .
$$

To obtain estimate (B.14), we repeatedly used the fact that the inequality

$$
\int_{y}^{\infty}(1+z)^{m} e^{-\frac{\alpha z^{2}}{2}} \mathrm{~d} z \lesssim(1+y)^{m-1} e^{-\frac{\alpha y^{2}}{2}}
$$

holds for $y \geq 0$ and $m \in \mathbb{R}$. 
The work that follows is different from [12]. Returning to (B.12), we integrate by parts three times in $y$ to find that

$$
e^{-\sigma L_{0}(\alpha)} \mathbb{P}\left[f_{0}\right](z)=-e^{\frac{\alpha z^{2}}{4}} \int \partial_{y}^{3} U_{0}(z, y) f^{(-3)}(y) \mathrm{d} y .
$$

Then we calculate directly and apply estimate (B.14) to obtain

$$
\begin{aligned}
&\left|\langle z\rangle^{-5} e^{\frac{\alpha z^{2}}{4}} e^{-\sigma L_{0}(\alpha)} \mathbb{P}_{3}\left[f_{0}\right](z)\right| \\
& \lesssim \frac{e^{-3 \alpha \sigma}}{\left(1-e^{-2 \alpha \sigma}\right)^{3}} e^{\frac{\alpha z^{2}}{2}}\langle z\rangle^{-5} \int(|z|+|y|+1)^{3} U_{0}(z, y)\left|f^{(-3)}(y)\right| \mathrm{d} y \\
& \lesssim \frac{e^{-3 \alpha \sigma}}{\left(1-e^{-2 \alpha \sigma}\right)^{3}}\langle z\rangle^{-5} e^{\frac{\alpha z^{2}}{2}}\left\|\langle z\rangle^{-5} e^{\frac{\alpha z^{2}}{4}} f_{0}\right\|_{L^{\infty}} \int(|z|+|y|+1)^{3} U_{0}(z, y) e^{-\frac{\alpha y^{2}}{2}}\left(1+|y|^{2}\right) \mathrm{d} y \\
& \lesssim \frac{e^{-3 \alpha \sigma}}{\left(1-e^{-2 \alpha \sigma}\right)^{3}}\left\|\langle z\rangle^{-5} e^{\frac{\alpha z^{2}}{4}} f_{0}\right\|_{L^{\infty}} \sum_{k=1,2,3,4,5}\langle z\rangle^{-k} e^{\frac{\alpha z^{2}}{2}} \int U_{0}(z, y) e^{-\frac{\alpha y^{2}}{2}}(1+|y|)^{k} \mathrm{~d} y .
\end{aligned}
$$

Here in the second step, we use the simple observation

$$
\langle z\rangle^{-5}(|z|+|y|+1)^{3}\left(1+|y|^{2}\right) \lesssim \sum_{k=1,2,3,4,5}\langle z\rangle^{-k}(1+|y|)^{k}
$$

to show that

$$
\sum_{k=1,2,3,4,5}\langle z\rangle^{-k} e^{\frac{\alpha z^{2}}{2}} \int U_{0}(z, y) e^{-\frac{\alpha y^{2}}{2}}(1+|y|)^{k} \mathrm{~d} y \lesssim e^{2 \alpha \sigma},
$$

Then we apply the same arguments as in the proof of Lemma 16 of [12], where the cases $k=1, \ldots, 4$ were verified. Because this adaptation is straightforward and simple, it does not need to be detailed here.

Now for $\sigma \geq 1$, estimates (B.15) and (B.16) together imply the desired decay estimate. On the other hand, for small $\sigma$, we apply (B.16) directly to (B.12) to obtain a uniform bound. This completes the proof.

\section{Appendix C. Proof of Lemma 12}

Proof of Lemma 12. We first derive evolution equations for $\xi_{ \pm}$and $\left(e^{-\frac{a y^{2}}{4}} \partial_{y} e^{\frac{a y^{2}}{4}} \xi\right)_{ \pm}$. To simplify notation, we introduce new functions $\psi_{ \pm}$defined by

$$
\psi_{ \pm}:=e^{-\frac{a y^{2}}{4}} \partial_{y}\left(e^{\frac{a y^{2}}{4}} \xi_{ \pm}\right) .
$$

Next we make the following observations.

Lemma 17. The functions $\xi_{ \pm}$and $\psi_{ \pm}$satisfy the orthogonality conditions

$$
\xi_{ \pm} \perp\left\{e^{-\frac{a y^{2}}{4}}, y e^{-\frac{a y^{2}}{4}}\right\} \quad \text { and } \quad \psi_{ \pm} \perp\left\{e^{-\frac{a y^{2}}{4}}\right\},
$$

and evolve by

$$
\begin{aligned}
& \partial_{\tau} \xi_{ \pm}=-L_{0}(a) \xi_{ \pm}+e^{-\frac{a y^{2}}{4}}\left[G_{1}+G_{2}\right], \\
& \partial_{\tau} \psi_{ \pm}=-\left[L_{0}(a)+a\right] \psi_{ \pm}+e^{-\frac{a y^{2}}{4}}\left[G_{3}+G_{4}\right],
\end{aligned}
$$

where

$$
L_{0}(a):=-\partial_{y}^{2}+\frac{a^{2}+\partial_{\tau} a}{4} y^{2}-\frac{3 a}{2} .
$$


The terms $G_{k}(k=1, \ldots, 4)$ on the RHS above satisfy the estimates

$$
\begin{array}{ll}
\left\|\langle y\rangle^{-2} G_{1}\right\|_{L^{\infty}} \lesssim \beta^{\frac{33}{10}}, & \left\|\langle y\rangle^{-3} G_{2}\right\|_{L^{\infty}} \lesssim \beta^{\frac{73}{20}}, \\
\left\|\langle y\rangle^{-1} G_{3}\right\|_{L^{\infty}} \lesssim \beta^{\frac{53}{20}}, & \left\|\langle y\rangle^{-2} G_{4}\right\|_{L^{\infty}} \lesssim \beta^{\frac{63}{20}} .
\end{array}
$$

The orthogonality conditions (C.1) follow directly from the corresponding properties for $\xi$. The remainder of the lemma is proved in Section C.1.

In what follows, we focus on estimating $\xi_{+}$. The remaining estimates are proved similarly. For $\xi_{+}$, after going through the same procedures as we followed when deriving (B.6), we obtain

$$
\partial_{\sigma}\left[\eta_{+}\right]=-L_{0}(\alpha)\left[\eta_{+}\right]+\tilde{G}_{1}(\sigma)+\tilde{G}_{2}(\sigma),
$$

where $L_{0}(\alpha)$ is the autonomous linear operator

$$
L_{0}(\alpha):=-\partial_{z}^{2}+\frac{\alpha^{2}}{4} z^{2}-\frac{3 \alpha}{2},
$$

and $\tilde{G}_{k}(k=1,2)$ are reparametrizations of $G_{k}(k=1,2)$ defined similarly to (B.7). The terms appearing in the evolution equation (C.3) satisfy the following estimates.

Lemma 18. If the assumptions of Section 6.1 hold, then for any $\tau \leq T_{1}$ and any weight $\ell \geq 0$, one has

$$
\left\|\langle z\rangle^{-\ell} e^{\frac{\alpha z^{2}}{4}} \eta_{+}(\sigma)\right\|_{L^{\infty}} \lesssim\left\|\langle y\rangle^{-\ell} e^{\frac{a y^{2}}{4}} \xi_{+}(\tau(\sigma))\right\|_{L^{\infty}},
$$

along with

$$
\left\|\langle z\rangle^{-2} \tilde{G}_{1}\right\|_{L^{\infty}} \lesssim \beta^{\frac{33}{10}}(\tau(\sigma)) \quad \text { and } \quad\left\|\langle z\rangle^{-3} \tilde{G}_{2}\right\|_{L^{\infty}} \lesssim \beta^{\frac{73}{20}}(\tau(\sigma))
$$

The proofs of estimates (C.4) and (C.5) are almost identical to those that appear in Lemma 9 above, hence are not repeated here.

Returning to equation (C.3), we apply Duhamel's principle to obtain

$$
\eta_{+}(\sigma)=e^{-\sigma L_{0}(\alpha)} \eta_{+}(0)+\int_{0}^{\sigma} e^{-\left(\sigma-\sigma_{1}\right) L_{0}(\alpha)}\left[\tilde{G}_{1}\left(\sigma_{1}\right)+\tilde{G}_{2}\left(\sigma_{1}\right)\right] \mathrm{d} \sigma_{1} .
$$

We again rely on a propagator estimate to prove the decay of $\eta_{+}$. Observe that the quantum harmonic oscillator $L_{\alpha}$ has nonpositive eigenvalues with eigenfunctions $e^{-\frac{\alpha}{4} z^{2}}$ and $z e^{-\frac{\alpha}{4} z^{2}}$, which might make $\eta$ grow. To control these eigenvectors, we use the orthogonality properties of $\eta_{+}$.

Recall (B.5) and note that at time $\sigma=\sigma\left(T_{1}\right)$, namely $\tau=T_{1}$, we have $\eta_{+}(\sigma)=$ $\xi_{+}\left(T_{1}\right)$ and $e^{\alpha z^{2} / 4}=e^{a(\tau) y^{2} / 4}$. Hence by (C.1), we have

$$
\mathbb{P}_{2}\left[\eta_{+}\right]\left(\sigma\left(T_{1}\right)\right)=\eta_{+}\left(\sigma\left(T_{1}\right)\right),
$$

where $\mathbb{P}_{2}$ denotes the orthogonal projection onto the subspace orthogonal to the span of $\left\{e^{-\alpha z^{2} / 4}, z e^{-\alpha z^{2} / 4}\right\}$ (i.e. orthogonal to the unstable subspace of $L_{\alpha}$ ). We apply $\mathbb{P}_{2}$ to both sides of (C.6), obtaining

$$
\begin{aligned}
\eta_{+}\left(\sigma\left(T_{1}\right)\right)= & \mathbb{P}_{2}\left[e^{-\sigma(T) L_{0}(\alpha)} \eta_{+}\right](0) \\
& +\int_{0}^{\sigma\left(T_{1}\right)} e^{-\left(\sigma(T)-\sigma_{1}\right) L_{0}(\alpha)} \mathbb{P}_{2}\left[\tilde{G}_{1}\left(\sigma_{1}\right)+\tilde{G}_{2}\left(\sigma_{1}\right)\right] \mathrm{d} \sigma_{1} \\
= & A_{1}+A_{2}+A_{3},
\end{aligned}
$$

where the terms $A_{1}, A_{2}, A_{3}$ are naturally defined.

We estimate $\mathbb{P}_{2} e^{-\sigma(T)} L_{0}(\alpha)$ as follows. 
Lemma 19. For all times that the assumptions of Section 6.1 hold, for any smooth function $g$, any such time $\sigma>0$, and any weight $k=2,3$, one has

$$
\left\|\langle z\rangle^{-k} e^{\frac{\alpha z^{2}}{4}} \mathbb{P}_{2}\left[e^{-\sigma L_{0}(\alpha)} g\right]\right\|_{L^{\infty}} \lesssim e^{-\alpha \sigma}\left\|\langle z\rangle^{-k} e^{\frac{\alpha z^{2}}{4}} g\right\|_{L^{\infty}} ;
$$

while for the chosen weight $\langle z\rangle^{-\frac{11}{10}}$, one has

$$
\left\|\langle z\rangle^{-\frac{11}{10}} e^{\frac{\alpha z^{2}}{4}} \mathbb{P}_{2}\left[e^{-\sigma L_{0}(\alpha)} g\right]\right\|_{L^{\infty}} \lesssim e^{-\frac{\alpha}{10} \sigma}\left\|\langle z\rangle^{-\frac{11}{10}} e^{\frac{\alpha z^{2}}{4}} g\right\|_{L^{\infty}} .
$$

Proof of Lemma 19. Both cases of Estimate (C.8) are proved by following the arguments in the proof of Lemma 16, mutatis mutandis.

Estimate (C.9) is obtained via an interpolation technique, as in Proposition 11.5 from [14]. Here, one interpolates between

$$
\begin{aligned}
\left\|\langle z\rangle^{-1} e^{\frac{\alpha z^{2}}{4}} \mathbb{P}_{2}\left[e^{-\sigma L_{0}(\alpha)} g\right]\right\|_{L^{\infty}} & =\left\|\langle z\rangle^{-1} e^{\frac{\alpha z^{2}}{4}} \mathbb{P}_{1}\left[e^{-\sigma L_{0}(\alpha)} \mathbb{P}_{2} g\right]\right\|_{L^{\infty}} \\
& \lesssim\left\|\langle z\rangle^{-1} e^{\frac{\alpha z^{2}}{4}} g\right\|_{L^{\infty}}
\end{aligned}
$$

and the $k=2$ case of estimate (C.8). Note that $\mathbb{P}_{1}$ denotes orthogonal projection onto the subspace orthogonal to $e^{-\frac{\alpha y^{2}}{4}}$. We omit further details.

Continuing with the proof of Lemma 12, we now apply (C.4) to estimate the first term $A_{1}$ in the decomposition (C.7) by

$$
\begin{aligned}
\left\|\langle z\rangle^{-\frac{11}{10}} e^{\frac{\alpha z^{2}}{4}} A_{1}\right\|_{L^{\infty}} & \lesssim e^{-\frac{\alpha}{10} \sigma(T)}\left\|\langle z\rangle^{-\frac{11}{10}} e^{\frac{\alpha z^{2}}{4}} \eta_{+}(0)\right\|_{L^{\infty}} \\
& \lesssim e^{-\frac{\alpha}{10} \sigma(T)}\left\|\langle y\rangle^{-\frac{11}{10}} e^{\frac{a y^{2}}{4}} \xi_{+}(0)\right\|_{L^{\infty}} \\
& \lesssim \beta^{\frac{53}{20}}\left(T_{1}\right) .
\end{aligned}
$$

Note that here we use the stronger ${ }^{13}$ restrictions $\left\|\langle x\rangle^{-\frac{11}{10}}\left(u_{0}\right)_{ \pm}\right\|_{L^{\infty}} \lesssim b^{\frac{53}{20}}(0)=$ $\beta^{\frac{53}{20}}(0)$ and $\left\|\langle x\rangle^{-\frac{11}{10}} \partial_{x}\left(u_{0}\right)_{ \pm}\right\|_{L^{\infty}} \lesssim b^{\frac{53}{20}}(0)=\beta^{\frac{53}{20}}(0)$ on the initial data contained in Assumption [A1] to get the estimate $\left\|\langle y\rangle^{-\frac{11}{10}} e^{\frac{a y^{2}}{4}} \xi_{ \pm}(0)\right\|_{L^{\infty}} \lesssim \beta^{\frac{53}{20}}(0)$ and the estimate $\left\|\langle y\rangle^{-\frac{11}{10}} \partial_{y} e^{\frac{a y^{2}}{4}} \xi_{ \pm}(0)\right\|_{L^{\infty}} \lesssim \beta^{\frac{53}{20}}(0)$. It follows that in the region $\beta y^{2} \leq 20$,

$$
\left|e^{\frac{\alpha z^{2}}{4}} A_{1}\right| \lesssim \beta^{\frac{21}{10}}\left(T_{1}\right)
$$

Here we also used the fact that $y=z$ at time $\tau=T_{1}$.

To estimate the second term in the decomposition (C.7), we use (C.5), obtaining

$$
\left\|\langle z\rangle^{-2} e^{\frac{\alpha z^{2}}{4}} A_{2}\right\|_{L^{\infty}} \lesssim \int_{0}^{\sigma(T)} e^{-\frac{1}{8}\left(\sigma\left(T_{1}\right)-\sigma_{1}\right)} \beta^{\frac{33}{10}}\left(\tau\left(\sigma_{1}\right)\right) \mathrm{d} \sigma \lesssim \beta^{\frac{33}{10}}\left(T_{1}\right) .
$$

Hence in the region $\beta y^{2} \leq 20$, one has

$$
\left|e^{\frac{\alpha z^{2}}{4}} A_{2}\right| \lesssim \beta^{\frac{23}{10}}\left(T_{1}\right)
$$

To estimate the final term $A_{3}$ in the decomposition (C.7), we use a different norm: here we apply (C.5) again to get

$$
\left\|\langle z\rangle^{-3} e^{\frac{\alpha z^{2}}{4}} A_{3}\right\|_{L^{\infty}} \lesssim \int_{0}^{\sigma\left(T_{1}\right)} e^{-\frac{1}{8}\left(\sigma\left(T_{1}\right)-\sigma_{1}\right)} \beta^{\frac{19}{5}}\left(\tau\left(\sigma_{1}\right)\right) \mathrm{d} \sigma \lesssim \beta^{\frac{19}{5}}\left(T_{1}\right) .
$$

\footnotetext{
${ }^{13}$ That is to say, stronger than we needed in [13].
} 
Thus in the region $\beta y^{2} \leq 20$, one has

$$
\left|e^{\frac{\alpha z^{2}}{4}} A_{3}\right| \lesssim \beta^{\frac{23}{10}}\left(T_{1}\right)
$$

Collecting estimates (C.10)-(C.12) above yields

$$
\left|e^{\frac{a(\tau) y^{2}}{4}} \xi_{+}\left(T_{1}\right)\right|=\left|e^{\frac{\alpha z^{2}}{4}} \eta_{+}\left(\sigma\left(T_{1}\right)\right)\right| \lesssim \beta^{\frac{23}{10}}\left(T_{1}\right) \quad \text { if } \quad \beta y^{2} \leq 20 .
$$

Because $T_{1} \geq 0$ is arbitrary, this completes the proof of the estimate for $\xi_{+}$in Lemma 12, modulo the proof of Lemma 17 that appears below. The remaining estimates are obtained in a wholly analogous manner.

\section{C.1. Proof of Lemma 17.}

Proof of Lemma 1\%. The proof is in two parts.

PART (I) We start by deriving the first evolution equation in (C.2) and its associated estimates. Instead of using equation (7.4) for $\xi$, it is more convenient to work directly from equation (2.3) for $v$, in order to see and exploit certain fortuitous cancellations.

Using notation defined in Section 2.1, we take an inner product $\left\langle e^{i \theta}, \cdot\right\rangle_{\mathbb{S}^{1}}$ with both sides of equation (2.3) to obtain

$$
\begin{aligned}
2 \pi \partial_{\tau} v_{+}= & \partial_{\tau}\left\langle e^{i \theta}, v\right\rangle_{\mathbb{S}^{1}} \\
= & \left\langle e^{i \theta}, F_{1}(p, q) \partial_{y}^{2} v\right\rangle_{\mathbb{S}^{1}}+\left\langle e^{i \theta}, v^{-2} F_{2}(p, q) \partial_{\theta}^{2} v\right\rangle_{\mathbb{S}^{1}} \\
& +\left\langle e^{i \theta}, v^{-1} F_{3}(p, q) \partial_{\theta} \partial_{y} v\right\rangle_{\mathbb{S}^{1}}+\left\langle e^{i \theta}, v^{-2} F_{4}(p, q) \partial_{\theta} v\right\rangle_{\mathbb{S}^{1}} \\
& -\left\langle e^{i \theta}, a y \partial_{y} v\right\rangle_{\mathbb{S}^{1}}+\left\langle e^{i \theta}, a v\right\rangle_{\mathbb{S}^{1}}-\left\langle e^{i \theta}, v^{-1}\right\rangle_{\mathbb{S}^{1}} .
\end{aligned}
$$

To transform this equation into a form similar to (C.2), we have to allocate appropriate terms to $G_{1}$ and $G_{2}$. For this purpose, we decompose various terms on the RHS of equation (C.13).

We decompose the first term on the RHS of (C.13) into two terms,

$$
\begin{aligned}
\left\langle e^{i \theta}, F_{1}(p, q) \partial_{y}^{2} v\right\rangle_{\mathbb{S}^{1}} & =\left\langle e^{i \theta}, \partial_{y}^{2} v\right\rangle_{\mathbb{S}^{1}}+\left\langle e^{i \theta},\left[F_{1}(p, q)-1\right] \partial_{y}^{2} v\right\rangle_{\mathbb{S}^{1}} \\
& =2 \pi \partial_{y}^{2} v_{+}+i\left\langle e^{i \theta}, \partial_{\theta}\left[\left(F_{1}(p, q)-1\right) \partial_{y}^{2} v\right]\right\rangle_{\mathbb{S}^{1}}
\end{aligned}
$$

with the final term above obtained by integrating by parts in $\theta$. Direct computation yields

$$
\begin{aligned}
\partial_{\theta}\left[\left(F_{1}(p, q)-1\right) \partial_{y}^{2} v\right]=-\frac{p^{2}}{1+p^{2}+q^{2}} & \partial_{\theta} \partial_{y}^{2} v \\
& -2 \frac{\partial_{y} \partial_{\theta} v \partial_{y} v}{1+p^{2}+q^{2}} \partial_{y}^{2} v+2 \frac{p^{2}\left(p \partial_{\theta} p+q \partial_{\theta} q\right)}{\left(1+p^{2}+q^{2}\right)^{2}} \partial_{y}^{2} v
\end{aligned}
$$


Now we group various terms on the RHS of (C.13) by introducing two functions $\tilde{G}_{1}$ and $\tilde{G}_{2}$, defined by

$$
\begin{aligned}
2 \pi \tilde{G}_{1}:= & \left\langle e^{i \theta},-\frac{2 \partial_{y} \partial_{\theta} v \partial_{y} v}{1+p^{2}+q^{2}} \partial_{y}^{2} v\right\rangle_{\mathbb{S}^{1}}+2\left\langle e^{i \theta}, \frac{p^{2}\left(p \partial_{\theta} p+q \partial_{\theta} q\right)}{\left(1+p^{2}+q^{2}\right)^{2}} \partial_{y}^{2} v\right\rangle_{\mathbb{S}^{1}} \\
& +\left\langle e^{i \theta}, v^{-2} F_{2}(p, q) \partial_{\theta}^{2} v\right\rangle_{\mathbb{S}^{1}}-\left\langle e^{i \theta}, v^{-1}\right\rangle_{\mathbb{S}^{1}} \\
& +\left\langle e^{i \theta}, v^{-1} F_{3}(p, q) \partial_{\theta} \partial_{y} v\right\rangle_{\mathbb{S}^{1}}+\left\langle e^{i \theta}, v^{-2} F_{4}(p, q) \partial_{\theta} v\right\rangle_{\mathbb{S}^{1}} \\
2 \pi \tilde{G}_{2}:= & -\frac{p^{2}}{1+p^{2}+q^{2}} \partial_{\theta} \partial_{y}^{2} v .
\end{aligned}
$$

This lets us write

$$
\partial_{\tau} v_{+}=\left[\partial_{y}^{2}-a y \partial_{y}+a\right] v_{+}+\tilde{G}_{1}+\tilde{G}_{2}
$$

where $\tilde{G}_{1}$ and $\tilde{G}_{2}$ satisfy the following estimates.

Lemma 20. For all times that the assumptions of Section 6.1 hold, one has

$$
\left\|\langle y\rangle^{-2} \tilde{G}_{1}\right\|_{L^{\infty}} \lesssim \beta^{\frac{33}{10}} \quad \text { and } \quad\left\|\langle y\rangle^{-3} \tilde{G}_{2}\right\|_{L^{\infty}} \lesssim \beta^{\frac{73}{20}} .
$$

Proof of Lemma 20. We first estimate $\tilde{G}_{2}$, computing directly to find that

$$
\left|\langle y\rangle^{-3} \frac{p^{2}}{1+p^{2}+q^{2}} \partial_{\theta} \partial_{y}^{2} v\right| \lesssim\langle y\rangle^{-2}|p|^{2}\langle y\rangle^{-1}\left|\partial_{\theta} \partial_{y}^{2} v\right| \lesssim \beta^{\frac{73}{20}} .
$$

In the final step above, we used the estimates $\langle y\rangle^{-1} \lesssim v^{-1}$ from (A.11) and $v^{-1}\left|\partial_{\theta} \partial_{y}^{2} v\right| \lesssim \beta^{\frac{33}{20}}$ from (6.9), as well as the estimate

$$
\begin{aligned}
\langle y\rangle^{-1}|p| \equiv\langle y\rangle^{-1}\left|\partial_{y} v\right| \leq\langle y\rangle^{-1}\left|\partial_{y} V_{a, b}\right| & \\
& +\sum_{k=0}^{4}\left|\beta_{k}\right|+\left|\partial_{y} \phi\right| \leq \beta+\beta^{\frac{11}{10}} M_{1,1} \lesssim \beta
\end{aligned}
$$

implied by the assumptions on $\beta_{k}(k=0, \cdots, 4)$ and the assumption $M_{1,1} \lesssim 1$.

Now we turn to $\tilde{G}_{1}$. The assumptions in (6.9), which are outputs of the first bootstrap machine, show that its first two terms admit the estimate

$$
\langle y\rangle^{-2}|\cdots| \lesssim \beta^{\frac{33}{10}}
$$

We treat the third and fourth terms of (C.13) together to exploit certain cancellations. By integrating by parts in $\theta$, we obtain

$$
\begin{aligned}
& \left\langle e^{i \theta}, v^{-2} F_{2}(p, q) \partial_{\theta}^{2} v\right\rangle_{\mathbb{S}^{1}}-\left\langle e^{i \theta}, v^{-1}\right\rangle_{\mathbb{S}^{1}} \\
& \quad=-i\left\langle e^{i \theta}, v^{-2} F_{2}(p, q) \partial_{\theta} v\right\rangle_{\mathbb{S}^{1}}+i\left\langle e^{i \theta}, v^{-2} \partial_{\theta} v\right\rangle_{\mathbb{S}^{1}}-\left\langle e^{i \theta}, \partial_{\theta}\left[v^{-2} F_{2}(p, q)\right] \partial_{\theta} v\right\rangle_{\mathbb{S}^{1}} \\
& \quad=-i\left\langle e^{i \theta}, v^{-2}\left[F_{2}(p, q)-1\right] \partial_{\theta} v\right\rangle_{\mathbb{S}^{1}}-\left\langle e^{i \theta}, \partial_{\theta}\left[v^{-2} F_{2}(p, q)\right] \partial_{\theta} v\right\rangle_{\mathbb{S}^{1}} .
\end{aligned}
$$

Recall that $F_{2}=\frac{1+p^{2}}{1+p^{2}+q^{2}}$. Controlling the terms that appear here is not hard, when one takes advantage of the presence of the operator $\partial_{\theta}$, the presence of sufficiently many factors of $v^{-1}$ (helped by the estimate $\langle y\rangle^{-1} \lesssim v^{-1}$ ), and employs estimates that have been proved and used frequently above. In this way, one finds that the third and fourth terms are bounded by

$$
\left\|\langle y\rangle^{-2}(\cdots)\right\|_{L^{\infty}} \lesssim \beta^{\frac{33}{10}} .
$$


In the same way, we find that the fifth and sixth terms of $\tilde{G}_{1}$ also admit the estimate

$$
\left\|\langle y\rangle^{-2}(\cdots)\right\|_{L^{\infty}} \lesssim \beta^{\frac{33}{10}} .
$$

Collecting the estimates above, completes the proof.

Returning to the proof of Lemma 17, we go back to equation (C.16). We must transform it further, because our objective is to derive an equation for $\xi_{+}$. The decomposition of $v$ in equation (6.1) implies that

$$
\begin{aligned}
\partial_{\tau} \xi_{+}=-L_{0}(a) \xi_{+}+e^{-\frac{a y^{2}}{4}} & \left.a-\partial_{\tau}\right]\left(\frac{1}{2} \beta_{1}+\frac{1}{2 i} \beta_{2}\right) \\
& -y e^{-\frac{a y^{2}}{4}} \partial_{\tau}\left(\frac{1}{2} \beta_{3}+\frac{1}{2 i} \beta_{4}\right)+e^{-\frac{a y^{2}}{4}} \tilde{G}_{1}+e^{-\frac{a y^{2}}{4}} \tilde{G}_{2} .
\end{aligned}
$$

To derive the simple form above, we repeatedly used the fact that $e^{-\frac{a y^{2}}{4}}$ and $y e^{-\frac{a y^{2}}{4}}$ are eigenvectors of $L_{0}(a)$. By the equations for $\beta_{k}(k=1, \ldots, 4)$ in Lemma 9 , we have

$$
\left|\left[-\partial_{\tau}+a\right]\left[\beta_{1}-i \beta_{2}\right]\right|+\left|\partial_{\tau}\left[\beta_{3}-i \beta_{4}\right]\right| \lesssim \beta^{\frac{33}{10}}
$$

This leads us to write

$$
\partial_{\tau} \xi_{+}=-L_{0}(a) \xi_{+}+G_{1}+G_{2}
$$

where

$$
G_{1}:=e^{-\frac{a y^{2}}{4}}\left[-\partial_{\tau}+a\right]\left(\frac{1}{2} \beta_{1}+\frac{1}{2 i} \beta_{2}\right)-e^{-\frac{a y^{2}}{4}} \partial_{\tau}\left(\frac{1}{2} \beta_{3} y+\frac{1}{2 i} \beta_{4} y\right)+e^{-\frac{a y^{2}}{4}} \tilde{G}_{1},
$$

and

$$
G_{2}:=e^{-\frac{a y^{2}}{4}} \tilde{G}_{2}
$$

To conclude the first part of the proof of Lemma 17, we apply Lemma 20 to obtain

$$
\left\|\langle y\rangle^{-2} e^{\frac{a y^{2}}{4}} G_{1}\right\|_{L^{\infty}} \lesssim \beta^{\frac{33}{10}} \quad \text { and } \quad\left\|\langle y\rangle^{-3} e^{\frac{a y^{2}}{4}} G_{2}\right\|_{L^{\infty}} \lesssim \beta^{\frac{73}{20}}
$$

PART (II) We now derive the second evolution equation in (C.2) and prove its associated estimates.

Our first task is to derive an evolution equation for $\left(\partial_{y} v\right)_{+}=\frac{1}{2 \pi}\left\langle e^{i \theta}, \partial_{y} v\right\rangle_{\mathbb{S}^{1}}$. (For $\left(\partial_{y} v\right)_{-}$, it suffices to observe that $\partial_{y} v_{-}=\overline{\left(\partial_{y} v\right)_{+}}$.) We compute directly to get

$$
\partial_{\tau} \partial_{y} v_{+}=\partial_{y}^{3} v_{+}-a y \partial_{y}^{2} v_{+}+\tilde{G}_{3}+\tilde{G}_{4}
$$

where

$$
\begin{aligned}
\tilde{G}_{3}:= & \left\langle e^{i \theta},\left[F_{1}(p, q)-1\right] \partial_{y}^{3} v\right\rangle_{\mathbb{S}^{1}}+\left\langle e^{i \theta}, \partial_{y} F_{1}(p, q) \partial_{y}^{2} v\right\rangle_{\mathbb{S}^{1}} \\
& +\left\langle e^{i \theta}, v^{-2}\left[F_{2}(p, q)-1\right] \partial_{y} \partial_{\theta}^{2} v\right\rangle_{\mathbb{S}^{1}}+\left\langle e^{i \theta}, \partial_{y}\left[v^{-2} F_{2}(p, q)\right] \partial_{\theta}^{2} v\right\rangle_{\mathbb{S}^{1}} \\
& +\left\langle e^{i \theta}, \partial_{y}\left[v^{-1} F_{3}(p, q) \partial_{\theta} \partial_{y} v\right]\right\rangle_{\mathbb{S}^{1}}+\left\langle e^{i \theta}, \partial_{y}\left[v^{-2} F_{4}(p, q) \partial_{\theta} v\right]\right\rangle_{\mathbb{S}^{1}}
\end{aligned}
$$

and

$$
\tilde{G}_{4}:=\left\langle e^{i \theta}, v^{-2} \partial_{\theta}^{2} \partial_{y} v\right\rangle_{\mathbb{S}^{1}}+\left\langle e^{i \theta}, v^{-2} \partial_{y} v\right\rangle_{\mathbb{S}^{1}} .
$$

We now proceed to estimate these quantities. 
To control $\tilde{G}_{4}$, we twice integrate by parts in $\theta$, obtaining

$$
\begin{aligned}
\tilde{G}_{4} & =\left\langle\partial_{\theta}^{2}\left[v^{-2} e^{i \theta}\right], \partial_{y} v\right\rangle_{\mathbb{S}^{1}}+\left\langle e^{i \theta}, v^{-2} \partial_{y} v\right\rangle_{\mathbb{S}^{1}} \\
& =\left\langle e^{i \theta} \partial_{\theta}^{2} v^{-2}+i e^{i \theta} \partial_{\theta} v^{-2}, \partial_{y} v\right\rangle_{\mathbb{S}^{1}} .
\end{aligned}
$$

Using the estimate $\left\|\langle y\rangle^{-1} \partial_{y} v\right\|_{L^{\infty}}=\mathcal{O}(\beta)$ coming from (C.18) and the estimate $v^{-2}\left|\partial_{\theta}^{2} v\right|=\mathcal{O}\left(\beta^{\frac{33}{20}}\right)$ coming from assumption (6.9), which is an output of the first bootstrap machine, we conclude that

$$
\left\|\langle y\rangle^{-1} \tilde{G}_{4}\right\|_{L^{\infty}} \lesssim \beta^{\frac{53}{20}} .
$$

To control $\tilde{G}_{3}$, we combine assumptions in (6.8) and (6.9) with the estimate for $\langle y\rangle^{-1}\left|\partial_{y} v\right|$ coming from (C.18) to get

$$
\left\|\langle y\rangle^{-2} \tilde{G}_{3}\right\|_{L^{\infty}} \lesssim \beta^{\frac{63}{20}}
$$

To conclude the second part of the proof, we again use the decomposition of $v$ in equation (6.1), this time to decompose the evolution equation for $\partial_{y} \psi_{+}$. The arguments used here are virtually identical to those which appear in part (I) of this proof. Hence we omit further details.

\section{REFERENCES}

[1] Angenent, Sigurd B.; Isenberg, James; Knopf, Dan. Formal matched asymptotics for degenerate Ricci flow neckpinches. Nonlinearity 24 (2011), 2265-2280.

[2] Angenent, Sigurd B.; Isenberg, James; Knopf, Dan. Degenerate neckpinches in Ricci flow. arXiv: $1208.4312 \mathrm{v} 1$.

[3] Angenent, Sigurd B.; Knopf, Dan. Precise asymptotics of the Ricci flow neckpinch. Comm. Anal. Geom. 15 (2007), no. 4, 773-844.

[4] Angenent, Sigurd B.; Velázquez, J. J. L. Degenerate neckpinches in mean curvature flow. J. Reine Angew. Math. 482 (1997), 15-66.

[5] Brendle, Simon; Huisken, Gerhard. Mean curvature flow with surgery of mean convex surfaces in $\mathbb{R}^{3}$. arXiv:1309.1461.

[6] Bricmont, Jean; Kupiainen, Antti. Universality in blow-up for nonlinear heat equations. Nonlinearity 7 (1994), no. 2, 539-575.

[7] Colding, Tobias Holck; Ilmanen, Tom; Minicozzi, William P., II. Rigidity of generic singularities of mean curvature flow. arXiv:1304.6356.

[8] Colding, Tobias Holck; Minicozzi, William P., II. Generic mean curvature flow I; generic singularities. Ann. of Math. (2) 175 (2012), no. 2, 755-833.

[9] Colding, Tobias Holck; Minicozzi, William P., II. Uniqueness of blowups and Łojaciewicz inequalities. arXiv:1312.4046v2.

[10] Daskalopoulos, P.; del Pino, Manuel. Type II collapsing of maximal solutions to the Ricci flow in $\mathbb{R}^{2}$. Ann. Inst. H. Poincaré Anal. Non Linéaire 24 (2007), no. 6, 851-874.

[11] Daskalopoulos, Panagiota; Šešum, Nataša. Type II extinction profile of maximal solutions to the Ricci flow in $\mathbb{R}^{2}$. J. Geom. Anal. 20 (2010), no. 3, 565-591.

[12] Dejak, Steven; Gang, Zhou; Sigal, Israel Michael; Wang, Shuangcai. Blow-up in nonlinear heat equations. Adv. in Appl. Math. 40 (2008), no. 4, 433-481.

[13] Gang, Zhou; Knopf, Dan; Sigal, Israel Michael. Neckpinch dynamics for asymmetric surfaces evolving by mean curvature flow. arXiv:1109.0939v2.

[14] Gang, Zhou; Sigal, Israel Michael. Neck pinching dynamics under mean curvature flow. J. Geom. Anal. 19 (2009), no. 1, 36-80.

[15] Haslhofer, Robert; Kleiner, Bruce. Mean curvature flow of mean convex hyper surfaces. arXiv: 1304.0926v2

[16] Haslhofer, Robert; Kleiner Bruce. Mean curvature flow with surgery. arXiv:1404.2332.

[17] Huisken, Gerhard. Asymptotic behavior for singularities of the mean curvature flow. J. Differential Geom. 31 (1990), no. 1, 285-299.

[18] Huisken, Gerhard; Sinestrari, Carlo. Convexity estimates for mean curvature flow and singularities of mean convex surfaces. Acta Math. 183 (1999), no. 1, 45-70. 
[19] Huisken, Gerhard; Sinestrari, Carlo. Mean curvature flow with surgeries of two-convex hypersurfaces. Invent. Math. 175 (2009), no. 1, 137-221.

[20] Ilmanen, Tom. Singularities of Mean Curvature Flow of Surfaces. (1995) Preprint. http://www.math.ethz.ch/ ilmanen/papers/pub.html

[21] Ilmanen, Tom. Lectures on mean curvature flow and related equations. (1995) Trieste notes.

[22] King, John R. Self-similar behavior for the equation of fast nonlinear diffusion. Philos. Trans. R. Soc., Lond., A, 343, (1993) 337-375.

[23] Schulze, Felix. Uniqueness of compact tangent flows in Mean Curvature Flow. J. Reine Angew. Math. To appear. (DOI:10.1515/crelle-2012-0070).

[24] Wang, Lu Uniqueness of self-similar shrinkers with asymptotically conical ends. J. Amer. Math. Soc. To appear. (DOI:10.1090/S0894-0347-2014-00792-X).

(Zhou Gang) California Institute of Technology

E-mail address: gzhou@caltech.edu

(Dan Knopf) University of Texas at Austin

E-mail address: danknopf@math.utexas.edu

$U R L:$ http://www.ma.utexas.edu/users/danknopf/ 